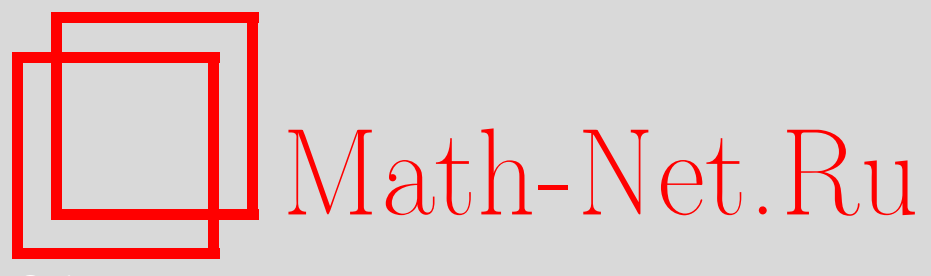

М. Маззокко, Л. О. Чехов, Орбифолдные римановы поверхности: пространства Тейхмюллера и алгебры геодезических функций, УМH, 2009, том 64, выпуск 6, 117168

DOI: https://doi.org/10.4213/rm9331

Использование Общероссийского математического портала Math-Net.Ru подразумевает, что вы прочитали и согласны с пользовательским соглашением http://www.mathnet.ru/rus/agreement

Параметры загрузки:

IP : 3.80 .253 .173

26 апреля 2023 г., 14:57:52

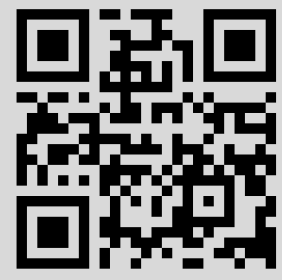




\title{
Орбифолдные римановы поверхности: пространства Тейхмюллера и алгебры геодезических функций
}

\begin{abstract}
М. Маззокко, Л. О. Чехов
Дается описание в терминах ленточных графов пространств Тейхмюллера римановых поверхностей с дырками и с $\mathbb{Z}_{2}$ - и $\mathbb{Z}_{3}$-орбифолдными точками (коническими особенностями) в униформизации Пуанкаре. Приведено соответствующее действие группы классов отображений, построены геодезические функции и введена пуассонова структура. Эта пуассонова структура впоследствии квантуется. В частных случаях поверхностей с $n$ $\mathbb{Z}_{2}$-орбифолдными точками и с одной или двумя дырками получены соответствующие $A_{n}$ - и $D_{n}$-алгебры геодезических функций (классические и квантовые). Бесконечномерная пуассонова алгебра $\mathfrak{D}_{n}$, которая представляет собой квазиклассический предел твистованной $q$-янгианной алгебры $Y_{q}^{\prime}\left(\mathfrak{o}_{n}\right)$ для ортогональной алгебры Ли $\mathfrak{o}_{n}$, ставится в соответствие алгебре геодезических функций на кольце с $n \mathbb{Z}_{2}$-орбифолдными точками. Представлено действие группы кос на этой алгебре. Этот результат применен при построении действия группы кос на конечномерных редукциях этой алгебры: редукции уровня $p$ и на алгебре $D_{n}$, что позволяет построить центральные элементы для этих редукций. Кроме того, алгебра $\mathfrak{D}_{n}$ интерпретируется как пуассонова алгебра данных монодромий фробениусова многообразия в окрестности неполупростой точки.

Библиография: 36 названий.
\end{abstract}

Ключевые слова: конические сингулярности, пространство модулей, алгебра геодезических, квантование.

\section{СОДЕРЖАНИЕ}

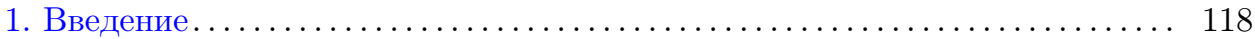

2. Ленточные графы и гиперболическая геометрия ................. 121

2.1. Гиперболическая геометрия при наличии вращений.......... 121

2.2. Геодезические функции на римановых поверхностях с $\mathbb{Z}_{2^{-}}$и $\mathbb{Z}_{3}$-орбифолдными точками...................... 124

2.3. Разбиение на идеальные треугольники и ленточные графы . . . . 125

Работа выполнена при поддержке грантов EPSRC ARF EP/D071895/1 и RA EP/ F03265X/1, РФФИ (гранты № 08-01-00501 и 09-01-92433-CE), программы "Ведущие научные школы" (грант НШ-795.2008.1), программы "Математические методы нелинейной динамики", Тренировочной сети им. Мари Кюри ENIGMA, Сети MISGAM Европейского научного фонда и Института математических исследований (Манчестер).

(C) М. МАззокко, Л.О. Чехов, 2009 
3. Преобразования группы классов отображений .................. 128

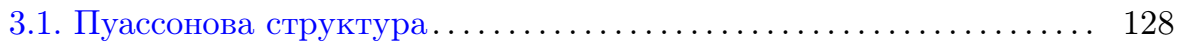

3.2. Морфизмы флипов на ленточных графах ................ 129

4. Алгебры геодезических функций ............................ 134

4.1. Структура геодезических и ламинаций на ленточных графах... 134

4.2. Пуассоновы скобки для геодезических функций .............. 134

5. Специальные случаи геодезических алгебр . . . . . . . . . . . . . . 136

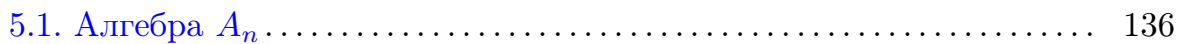

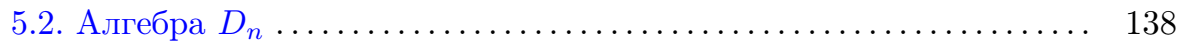

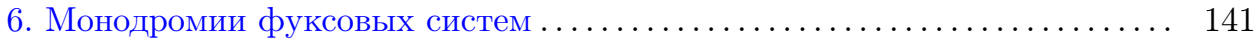

6.1. Действие группы кос............................. 142

6.2. Скобка Короткина-Самтлебена .......................... 142

6.3. Процедура слияния полюсов ......................... 143

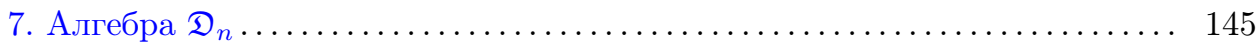

7.1. Пример: слияние двух полюсов ..................... 147

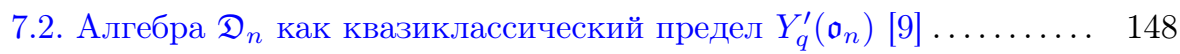

7.3. Соотношения группы кос для $\mathfrak{D}_{n} \ldots \ldots \ldots \ldots \ldots \ldots \ldots \ldots \ldots \ldots \ldots . \ldots \ldots$

8. Центральные элементы алгебр $\mathfrak{D}_{n}$ и их пуассоновых редукций........ 151

8.1. Центральные элементы алгебры $A_{n} \ldots \ldots \ldots \ldots \ldots \ldots \ldots \ldots \ldots . \ldots \ldots$

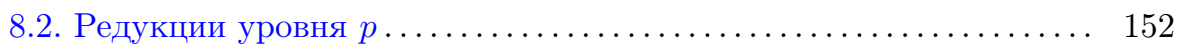

8.3. Алгебра $D_{n}$ как редукция алгебры $\mathfrak{D}_{n} \ldots \ldots \ldots \ldots \ldots \ldots \ldots \ldots \ldots$

9. Квантовые пространства Тейхмюллера орбифолдных римановых по-

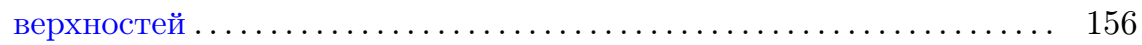

9.1. Каноническое квантование алгебр Пуассона............... 156

9.2. Квантовые геодезические функции . . . . . . . . . . . . . . . 156

9.3. Квантовые распутывающие соотношения ................ 157

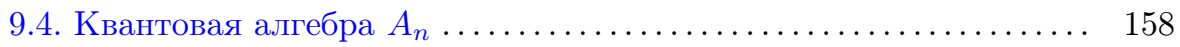

9.5. Действие квантовой группы кос на твистованном янгиане ..... 160

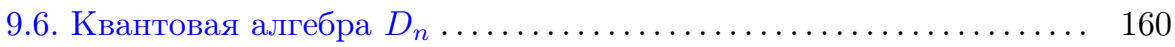

10. Фробениусовы многообразия в окрестности неполупростой точки.... 163

10.1. Слияние полюсов для фробениусова многообразия........... 164

10.2. Редукции уровня $p$ в случае фробениусовых многообразий . . . 165

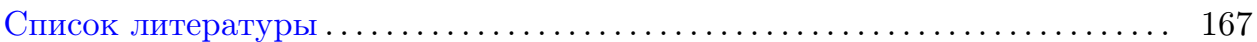

\section{1. Введение}

В последнее десятилетие теория Тейхмюллера привлекает к себе интерес сообщества математических физиков ввиду того, что пространства Тейхмюллера проявляются в качестве гильбертовых пространств трехмерной квантовой гравитации [1]. Пространства Тейхмюллера допускают каноническую пуассонову структуру (скобку Вейля-Петерсона) и обладают группами симметрий, которые представляют собой группы классов отображений гомеоморфизмов, сохраняющих ориентацию поверхности. Алгебра наблюдаемых представляет собой совокупность функций длин геодезических, представляющих гомотопические классы замкнутых кривых. 
Алгебры функций геодезических длин, возникающие при исследовании пространств Тейхмюллера гиперболических римановых поверхностей, тесно связаны с алгебрами, возникающими при исследовании изомонодромных задач. Например, алгебра Нельсон-Редже [2], [3], появившаяся как алгебра геодезических функций на римановой поверхности рода $g$ с одной или двумя дырками [4], [5], равно как и изоморфная ей алгебра $A_{n}$ геодезических функций на диске с $n$ орбифолдными точками [6], совпадают с пуассоновыми алгебрами монодромий в фуксовых системах, возникающих в теории фробениусовых многообразий [7] и с алгебрами группоида верхнетреугольных матриц [8]. Это совпадение между алгебрами до сих пор остается во многом непонятым. В [9] была дана характеристика естественной изомонодромной связности на проколотой плоскости $\mathbb{P}^{1}$ (связность Черна-Саймонса), группа монодромий которой задается фуксовой группой на диске с $n \mathbb{Z}_{2}$-орбифолдными точками. Это показывает, что алгебры $A_{n}$ совпадают с пуассоновыми алгебрами данных монодромий для фуксовой системы размерности $2 \times 2$ с $n+1$ полюсами.

Обобщив это соответствие, авторы ввели новый тип пуассоновых алгебр, которые геометрически происходят из алгебр геодезических функций на кольце с $n \mathbb{Z}_{2}$-орбифолдными точками. Соответствующую фуксову систему можно получить, если начать с системы $A_{n+m}$ и провести процедуру слияния $m$ регулярных особенностей, что приводит к возникновению новой дырки. Получаемая при этом алгебра данных монодромий оказывается квадратичной алгеброй, не зависящей от числа $m$ объединяемых полюсов, и ее можно интерпретировать как абстрактную алгебру $\mathfrak{D}_{n}$ с бесконечным количеством генераторов $G_{i, j}^{(k)}$, $i, j=1, \ldots, n$, распределенных по уровням, нумеруемым индексом $k \in \mathbb{Z}_{\geqslant 0}$ (см. теорему 7.1). Эта алгебра $\mathfrak{D}_{n}$ оказывается квазиклассическим пределом твистованной $q$-янгианной алгебры $Y_{q}^{\prime}\left(\mathfrak{o}_{n}\right)$ для ортогональной алгебры Ли $\mathfrak{o}_{n}$ [10], или, другими словами, определяющие соотношения алгебры $\mathfrak{D}_{n}$ оказываются квазиклассическим пределом хорошо известного уравнения отражений.

Помимо пуассоновой структуры другим общим свойством алгебр геодезических функций на пространстве Тейхмюллера оказываются соотношения группы кос, которые порождают группу классов отображений. Инварианты относительно действия группы кос одновременно оказываются центральными элементами пуассоновой алгебры, а потому построение удобного представления для группы кос всегда оказывается полезным при решении задач о построении центральных элементов пуассоновых алгебр. Такое представление в терминах присоединенного матричного действия для алгебр $A_{n}$ было найдено в работе [11], и оно в дальнейшем было использовано [8] при построении пуассоновых инвариантов соответствующих алгебр.

В работе [9] действие генераторов группы кос на алгебре $\mathfrak{D}_{n}$ было представлено в виде присоединенного матричного действия (см. предложение 7.6). В силу топологических соображений это действие порождается $n$ генераторами, среди которых генераторы $\beta_{i, i+1}, i=1, \ldots, n-1$, переставляют орбифолдные точки с номерами $i$ и $(i+1)$, а новый генератор $\beta_{n, 1}$ переставляет первую и последнюю орбифолдные точки и перемешивает различные уровни $k$.

Имеются два характерных типа конечномерных пуассоновых редукций, для которых удается построить явные выражения для производящих функций их 
центральных элементов. Редукция на уровне $p$ отвечает возникновению новой орбифолдной точки порядка $p$ вместо новой дырки, а редукuия $\kappa$ алгебре $D_{n}$ представляет собой редукцию к конечно порожденной кубической алгебре, найденной в работе [12], где также было построено соответствующее действие группы кос. Отталкиваясь от квантового представления для действия группы кос для квантовой алгебры $D_{n}$, полученного в [12], [6], удается построить квантовое действие группы кос в матричном виде также и для алгебры $\mathfrak{D}_{n}$, равно как и для ее (квантовых) редукций уровня $p$. После этого можно уже построить полное действие квантовой группы кос на твистованной квантовой обертывающей алгебре $U_{q}^{\prime}\left(\mathfrak{o}_{n}\right)[13]$.

Наконец, мы интерпретируем построенную алгебру $\mathfrak{D}_{n}$ как пуассонову алгебру данных Стокса на фробениусовых многообразиях в окрестности неполупростой точки.

Работа организована следующим образом. В разделе 2 представлены новые результаты относительно введения $\mathbb{Z}_{3}$-орбифолдных точек в комбинаторное описание пространств Тейхмюллера римановых поверхностей с дырками и с $\mathbb{Z}_{2}$-орбифолдными точками и относительно построения скобок Голдмана [14] для соответствующих геодезических функций. Соответствующие преобразования группы классов отображений, задаваемые морфизмами графов, представлены в разделе 3 . В разделе 4 рассматриваются алгебры геодезических функций и ламинации на общей римановой поверхности, а в разделе 5 эти конструкции ограничиваются на поверхности конкретного типа, геодезические алгебры которых появляются при исследовании алгебр данных монодромий фуксовых систем и при изучении алгебр твистованных янгианов. Специальный случай алгебры Нельсон-Редже рассматривается в п. 5.1, а случай алгебр $D_{n}$ содержится в п. 5.2. В разделе 6 рассматриваются изомонодромные деформации фуксовых систем с $n+1$ полюсами и вводятся соответствующие данные монодромии. Скобки Пуассона на множестве этих данных монодромии задаются скобками Короткина-Самтлебена [15], которые рассматриваются в п. 6.2. В п. 6.3 строится процедура слияния полюсов, которая применяется при построении новой дырки. Новые (бесконечномерные) пуассоновы алгебры $\mathfrak{D}_{n}$ данных монодромий на кольце с $n \mathbb{Z}_{2}$-орбифолдными точками вводятся в разделе 7 , где также показано, что алгебры $\mathfrak{D}_{n}$ представляют собой квазиклассический предел твистованных $q$-янгианных алгебр $Y_{q}^{\prime}\left(\mathfrak{o}_{n}\right)$ для алгебры Ли $\mathfrak{o}_{n}$ ортогональных матриц. В п. 7.3 построено представление действия группы кос в матричном виде. Различные редукции этих алгебр исследуются в разделе 8 , где вводятся редукции уровня $p$, т. е. алгебры $\mathfrak{D}_{n}^{(p)}$, и вычисляются их центральные элементы. Показано, что алгебра $D_{n}$ представляет собой специальную редукцию (основанную на применении распутывающих соотношений) из "объемлющей" алгебры $\mathfrak{D}_{n}$, а потому она также допускает то же самое представление действия группы кос в классическом случае.

Квантовые алгебры геодезических рассматриваются в разделе 9. Там представлено действие квантовой группы кос на квантовую версию алгебры $\mathfrak{D}_{n}$, которая и представляет собой твистованную $q$-янгианную алгебру $Y_{q}^{\prime}\left(\mathfrak{o}_{n}\right)$. Такое же матричное представление остается справедливым для всех ее (квантовых) редукций уровня $p$, однако построение квантового действия группы кос 
на элементах квантовых алгебр $D_{n}$ требует возврата к конструкции работы [6], основанной на наблюдении, что любая редукция уровня $p$ при $p>2$ согласована с $D_{n}$-редукцией. Связь с теорией фробениусовых многообразий производится в разделе 10 .

\section{2. Ленточные графы и гиперболическая геометрия}

\section{1. Гиперболическая геометрия при наличии вращений.}

2.1.1. Описание в терминах ленточных графов римановых поверхностей $c$ дырками. Начнем с краткого напоминания того, как фуксова группа $\Delta_{g, s}$, которая представляет собой конечно порожденную дискретно действующую подгруппу группы $\operatorname{PSL}(2, \mathbb{R})$, может быть комбинаторно описана в терминах ленточных графов. В отсутствие орбифолдных точек соответствующий ленточный граф представляет собой трехвалентный граф (каждая вершина инцидентна ровно трем концам неориентированных ребер; два конца одного ребра могут встречаться в одной и той же вершине), ребра которого занумерованы несовпадающими целыми числами $\alpha=1,2, \ldots, 6 g-6+3 s$, а в каждой вершине задан предписанный циклический порядок вхождения ребер в эту вершину. Такой граф представляется естественным образом, если его нарисовать без самопересечений на ориентированной римановой поверхности с дырками.

ОПРЕДЕЛЕНИЕ 2.1. Трехвалентный ленточный граф $\Gamma_{g, s}$ называется остовом римановой поверхности $\Sigma_{g, s}$ рода $g$ с $s$ дырками $(s \geqslant 1,2 g-2+s>0)$, если ленточный граф $\Gamma_{g, s}$ можно вложить без самопересечений в эту риманову поверхность таким образом, чтобы его дополнение представляло собой несвязный набор граней, каждая из которых есть многоугольник, содержащий внутри себя ровно одну дырку, при заклеивании которой грань становится односвязной.

Первые гомотопические группы $\pi_{1}\left(\Sigma_{g, s}\right)$ и $\pi_{1}\left(\Gamma_{g, s}\right)$ совпадают ввиду того, что каждый замкнутый путь на поверхности $\Sigma_{g, s}$ можно единственным способом гомотопически эквивалентно преобразовать в замкнутый путь на графе $\Gamma_{g, s}$ (всюду в дальнейшем предполагается, что пути не испытывают разворотов на ребрах и в вершинах). Стандартный результат гиперболической геометрии при этом утверждает, что классы сопряженности элементов фуксовой группы $\Delta_{g, s}$ находятся во взаимно однозначном соответствии с гомотопическими классами замкнутых путей на римановой поверхности $\Sigma_{g, s}=\mathbb{H}_{+}^{2} / \Delta_{g, s}$ и что длина $\ell_{\gamma}$ гиперболического элемента $\gamma \in \Delta_{g, s}$ совпадает с минимальной длиной кривых из соответствующего гомотопического класса: эта длина и есть длина единственной замкнутой геодезической, принадлежащей данному классу.

Стандартный набор генераторов первой гомотопической группы $\pi_{1}\left(\Sigma_{g, s}\right)$ включает в себя $2 g$ элементов $A_{i}, B_{i}, i=1, \ldots, g$, отвечающих обходам вокруг циклов $a_{i}, b_{i}$ на римановой поверхности (со стандартными условиями пересечений $\left.a_{i} \circ b_{j}=\delta_{i j}, a_{i} \circ a_{j}=b_{i} \circ b_{j}=0\right)$, и $s$ генераторов $P_{j}, j=1, \ldots, s$, отвечающих обходам вокруг дырок (в одном и том же направлении относительно ориентации римановой поверхности) с единственным условием

$$
A_{1} B_{1} A_{1}^{-1} B_{1}^{-1} A_{2} B_{2} A_{2}^{-1} B_{2}^{-1} \cdots A_{g} B_{g} A_{g}^{-1} B_{g}^{-1} P_{1} \cdots P_{s}=\mathbb{E},
$$


из которого следует, что полное число свободных параметров равно $6 g+3 s-6$ (три параметра фиксируются вышеприведенным условием, а три остальных отвечают свободе глобального сопряжения всех матриц).

Оказывается, что это условие достаточно непросто применить на практике; преимущество комбинаторного подхода, излагаемого ниже, заключается в том, что пуассоновы скобки оказываются очень простыми на уровне координат пространства Тейхмюллера, а потому их центральные элементы (обходы вокруг дырок) также допускают простое представление.

Комбинаторное описание пространств Тейхмюллера использует приведенное выше взаимно однозначное соответствие между классами сопряженности фуксовой группы и замкнутыми путями на соответствующем остовном графе. Поставим в соответствие ребру с номером $\alpha$ вещественное число $Z_{\alpha}$ и будем подставлять [16] матрицу преобразования Мёбиуса

$$
X_{Z_{\alpha}}=\left(\begin{array}{cc}
0 & -e^{Z_{\alpha} / 2} \\
e^{-Z_{\alpha} / 2} & 0
\end{array}\right)
$$

каждый раз, когда путь, гомеоморфный геодезической $\gamma$, будет проходить через ребро $\alpha$.

Определим также матрицы “правых" и “левых" поворотов, помещаемые в соответствующее место в матричном произведении каждый раз, когда путь совершает соответствующий поворот в вершине:

$$
R=\left(\begin{array}{cc}
1 & 1 \\
-1 & 0
\end{array}\right), \quad L=R^{2}=\left(\begin{array}{cc}
0 & 1 \\
-1 & -1
\end{array}\right) .
$$

Элемент фуксовой группы при этом имеет структуру

$$
P_{\gamma}=L X_{Z_{n}} R X_{Z_{n-1}} \cdots R X_{Z_{2}} R X_{Z_{1}},
$$

а соответствующая геодезическая функиия

$$
G_{\gamma} \equiv \operatorname{Tr} P_{\gamma}=2 \cosh \frac{\ell_{\gamma}}{2}
$$

связана с настоящей длиной $\ell_{\gamma}$ замкнутой геодезической на римановой поверхности.

Полное число параметров в данном случае равно числу ребер остовного графа $\Gamma_{g, s}$, которое в соответствии с формулой Эйлера в точности равно искомому числу $6 g-6+3 s$.

ЗАмЕчАниЕ 2.2. Заметим, что матричные комбинации

$$
R X_{z}=\left(\begin{array}{cc}
e^{-Z / 2} & -e^{Z / 2} \\
0 & e^{Z / 2}
\end{array}\right) \quad \text { и } \quad L X_{z}=\left(\begin{array}{cc}
e^{-Z / 2} & 0 \\
-e^{-Z / 2} & e^{Z / 2}
\end{array}\right)
$$

равно как и любые произведения (3) произвольного числа таких матриц, имеют знаковую структуру $\left(\begin{array}{ll}+ & - \\ - & +\end{array}\right)$, а след произведения $P_{\gamma}$ при этом всегда представляется суммой экспоненциальных мономов с положительными целыми коэффициентами; в этой сумме всегда присутствуют члены $e^{Z_{1} / 2+\cdots+Z_{n} / 2}$ 
и $e^{-Z_{1} / 2-\cdots-Z_{n} / 2}$, а потому вся сумма всегда не меньше двух. Таким образом, соответствующий элемент фуксовой группы всегда или гиперболичен, или параболичен, причем последнее возможно, только если $Z_{1}+\cdots+Z_{n}=0$ и все матрицы поворотов в формуле (3) равны или $R$ или $L$, что отвечает пути, идущему вдоль границы грани; все такие пути гомеоморфны периметрам дырок, а условие, что сумма $Z_{i}$ вдоль такого пути равна нулю, обозначает вырождение дырки в пунктированную точку.

2.1.2. Обобщение конструкции на поверхности $c \mathbb{Z}_{2^{-}}$и $\mathbb{Z}_{3}$-орбифолдными точками. Новыми генераторами фуксовой группы будут повороты на углы $\pi$ и $2 \pi / 3$ в соответствующих конечных наборах $\delta^{(1)}$ и $\delta^{(2)}$ точек на римановой поверхности ${ }^{1}$. Генераторы $F_{i}, i=1, \ldots,\left|\delta^{(1)}\right|$, поворотов на угол $\pi$ сопряжены одной и той же матрице

$$
F_{i}=U_{i} F U_{i}^{-1}, \quad F=\left(\begin{array}{cc}
0 & 1 \\
-1 & 0
\end{array}\right),
$$

а повороты на угол $2 \pi / 3$ сопряжены матрице $L(2), Q_{j}=U_{j} L U_{j}^{-1}, j=$ $1, \ldots,\left|\delta^{(2)}\right|$.

Пополнение стандартного набора генераторов (сдвиги вдоль $a$ - и $b$-циклов и обходы вокруг дырок) новыми элементами, отвечающими поворотам, не всегда приводит к регулярной (метризуемой) римановой поверхности, поскольку действие получающейся при этом группы не обязательно оказывается дискретным. Необходимые и достаточные условия того, что добавление новых генераторов приведет к образованию регулярной поверхности, снова могут быть сформулированы в терминах графов (см. [17] относительно случая $\mathbb{Z}_{2}$-орбифолдных точек) ${ }^{2}$.

В случаях, когда мы не делаем различия между типами орбифолдных точек, используется обозначение без верхнего индекса. Таким образом, будем полагать $\delta=\delta^{(1)} \cup \delta^{(2)}$.

Расширим теперь понятие ленточного графа [18] на случай орбифолдных римановых поверхностей. Занумеруем сначала все орбифолдные точки несовпадающими целыми числами $1, \ldots,|\delta|$. Введем дополнительную маркировку (точки и звезды) на множестве $\delta$, чтобы различать соответствующие $\mathbb{Z}_{2}$ и $\mathbb{Z}_{3}$-орбифолдные точки. Затем разобьем произвольным образом набор всех орбифолдных точек $\delta$ на $s$ непересекающихся (возможно, пустых) подмножеств $\delta_{k},\left|\delta_{k}\right| \geqslant 0, \bigcup_{k=1}^{s} \delta_{k}=\delta$, каждое из которых может содержать орбифолдные точки обоих типов. Подмножеству $\delta_{k}$ поставим в соответствие (в смысле теории множеств) дырку с номером $k$ и введем также циклическое упорядочение внутри каждого набора $\delta_{k}$.

ОПРЕДЕлЕНИЕ 2.3. Будем называть ленточный граф $\Gamma_{g, s,\left|\delta^{(1)}\right|,\left|\delta^{(2)}\right|}$ остовом римановой поверхности $\Sigma_{g, s, \delta}$ рода $g$ с $s$ дырками, с $\left|\delta^{(1)}\right| \mathbb{Z}_{2}$-орбифолдными точками и с $\left|\delta^{(2)}\right| \mathbb{Z}_{3}$-орбифолдными точками, если

(а) этот граф вкладывается без самопересечений в $\Sigma_{g, s, \delta}$,

\footnotetext{
${ }^{1}$ Символом $|\cdot|$ будем обозначать мощность соответствующего множества.

${ }^{2} \mathrm{~B}$ дальнейшем риманова поверхность называется регулярной, если она представляет собой локально гладкую поверхность постоянной отрицательной кривизны всюду, кроме в точности $\left|\delta^{(1)} \cup \delta^{(2)}\right|$ орбифолдных точек.
} 
(b) все вершины графа $\Gamma_{g, s,\left|\delta^{(1)}\right|,\left|\delta^{(2)}\right|}$ имеют валентность три, кроме в точности $|\delta|$ одновалентных вершин (концов “висячих" ребер), расположенных в соответствующих орбифолдных точках,

(c) дополнение к графу $\Gamma_{g, s,\left|\delta^{(1)}\right|,\left|\delta^{(2)}\right|}$ на римановой поверхности $\Sigma_{g, s, \delta}$ представляет собой несвязный набор из $s$ многоугольников, каждый из которых содержит в точности одну дырку и становится односвязным при заклеивании этой дырки,

(d) каждой $\mathbb{Z}_{2}$-орбифолдной точке ставится в соответствие одновалентная вершина, отмеченная жирной точкой, а каждой $\mathbb{Z}_{3}$-орбифолдной точке ставится в соответствие одновалентная вершина, отмеченная звездой.

Построим теперь остовный граф $\Gamma_{g, s,\left|\delta^{(1)}\right|,\left|\delta^{(2)}\right|}$, отвечающий вышеприведенному разбиению множества $\delta$ на подмножества $\delta_{k}$. Для этого заметим, что каждое висячее ребро заканчивается в орбифолдной точке, относящейся к некоторому подмножеству $\delta_{k}$ и что это ребро вдается внутрь $k$-й граничной компоненты (дырки) (см. рис. 1 и примеры на других рисунках). Остовный граф $\Gamma_{g, s,\left|\delta^{(1)}\right|,\left|\delta^{(2)}\right|}$ должен быть таким, чтобы это была именно та дырка, которая связана с подмножеством $\delta_{k}$. При этом имеет место естественный циклический порядок одновалентных вершин, ассоциированных с $k$-й граничной компонентой графа $\Gamma_{g, s,\left|\delta^{(1)}\right|,\left|\delta^{(2)}\right|}$, который должен совпадать с предписанным циклическим упорядочением вершин в подмножестве $\delta_{k}$.

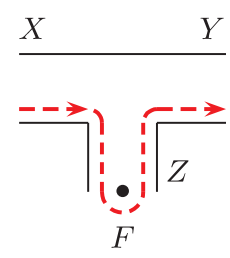

(a)

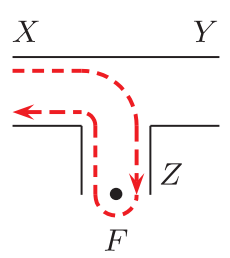

(b)

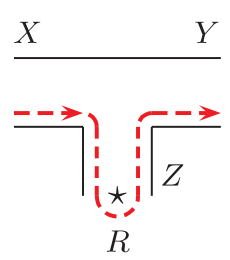

(c)

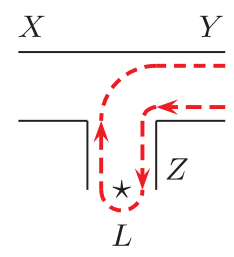

(d)

Рис. 1. Часть графа с висячим ребром. Его конец с орбифолдной точкой направлен вовнутрь граничной компоненты, с которой ассоциирована данная точка. Висячему ребру отвечает переменная $Z$. На рисунке показаны четыре типичных примера геодезических, испытывающих повороты в $\mathbb{Z}_{2-}$ и $\mathbb{Z}_{3}$-орбифолдных точках.

В результате получим ленточный граф данного рода $g$ с заданным числом дырок (пунктированных точек) $s$ и с заданным числом $|\delta|$ висячих ребер, среди которых $\left|\delta^{(1)}\right|$ ребер заканчиваются в точечных вершинах и $\left|\delta^{(2)}\right|$ ребер заканчиваются в звездчатых вершинах. Снабдим теперь все ребра этого графа, включая висячие ребра (полное число ребер равно $6 g-6+3 s+2|\delta|$ ), вещественными параметрами $Z_{\alpha}$. (В случае $\mathbb{Z}_{2}$-орбифолдных точек такая картина была впервые предложена В.В. Фоком и А.Б. Гончаровым [19]; соответствующие морфизмы графов были описаны в [6].)

2.2. Геодезические функции на римановых поверхностях с $\mathbb{Z}_{2}$ и $\mathbb{Z}_{3}$-орбифолдными точками. Предложим теперь удобную параметризацию геодезических кривых, отвечающих элементам фуксовой группы. Геоде- 
зическая линия испытывает поворот, когда она обходит вокруг точечной или звездчатой вершины; в случае $\mathbb{Z}_{2}$-орбифолдной точки при этом в соответствующую цепочку $(2 \times 2)$-матриц подставляется матрица $F(5)$, а в случае $\mathbb{Z}_{3}$-орбифолдной точки подставляется или матрица $R$ или матрица $L(2)$, в зависимости от направления обхода вокруг орбифолдной точки: против или по часовой стрелке. Например, части геодезических функций для четырех случаев, представленных на рис. 1, имеют вид:

$$
\begin{aligned}
& \text { (a) } \ldots X_{Y} R X_{Z} F X_{Z} R X_{X} \ldots, \\
& \text { (b) } \ldots X_{Y} L X_{Z} F X_{Z} R X_{X} \ldots, \\
& \text { (c) } \ldots X_{Y} R X_{Z} R X_{Z} R X_{X} \ldots, \\
& \text { (d) } \ldots X_{Y} R X_{Z} L X_{Z} L X_{Y} \ldots
\end{aligned}
$$

Отметим простые соотношения

$$
X_{Z} F X_{Z}=X_{2 Z}, \quad R=L+\mathbb{E}, \quad X_{Z}^{2}=-\mathbb{E} .
$$

Из замечания 2.2 следует, что все таким образом построенные геодезические функции в конечном счете имеют вид (3) (с удвоением некоторых из параметров $Z_{i}$ ). Все эти геодезические функции отвечают, тем самым, гиперболическим или параболическим элементам (последнее возможно только в случае геодезических, обходящих вокруг дырок, и только если дырка вырождается в пунктированную точку). Единственными эллиптическими элементами оказываются в точности элементы, сопряженные $F_{i}$ и $Q_{j}$; в последнем случае соответствующие геодезические функции равны нулю $\left(\operatorname{Tr} F_{i}=0\right)$ или единице $\left(\left|\operatorname{Tr} Q_{j}\right|=\operatorname{Tr} R=|\operatorname{Tr} L|=1\right)$.

Метризуемая риманова поверхность, тем самым, получается при произвольном выборе вещественных параметров $Z_{\alpha}$, связанных с ребрами остовного графа; основная лемма работы [6], сформулированная в случае $\mathbb{Z}_{2}$-орбифолдных точек, утверждает, что обратное также верно: любая метризуемая риманова поверхность может быть получена таким образом. Доказательство, содержащееся в работе [6], несет конструктивный характер и может быть непосредственно обобщено на случай орбифолдных точек произвольного типа, так что в дальнейшем декорированные пространства Тейхмюллера $\mathscr{T}_{g, s, \delta}^{H}[16]$ римановых поверхностей с орбифолдными точками будут отождествляться с пространствами $\mathbb{R}^{6 g-6+3 s+2|\delta|}$ вещественных параметров, ассоциированных с ребрами остовного графа $\Gamma_{g, s,\left|\delta^{(1)}\right|,\left|\delta^{(2)}\right| \cdot}$

Также имеет место теперь уже вполне очевидное утверждение о полиномиальности геодезических функций.

ПреДЛОЖЕНИЕ 2.4. Все $G_{\gamma}$, построенные с помощъю конструкиии (4), представляют собой лорановские многочлены от переменных $e^{Z_{i} / 2}$ с положительными целыми коэффициентами.

\section{3. Разбиение на идеальные треугольники и ленточные графы.} Приведем теперь геометрическую картину, лежащую в основе нашей алгебраической конструкции, а именно разбиение на идеалъные треугольники [18] для орбифолдных римановых поверхностей. 
Пусть дана регулярная риманова поверхность рода $g$ с $s$ дырками и с $\left|\delta_{k}\right|$ $\mathbb{Z}_{2^{-}}$и $\mathbb{Z}_{3}$-орбифолдными точками, ассоциированными с дыркой с номером $k$ и циклически упорядоченными внутри подмножества $\delta_{k}$. Выпустим из каждой орбифолдной точки геодезическую (единственную в заданном гомотопическом классе), которая навивается в заданном направлении (одном и том же для всех геодезических линий разбиения, навивающихся на данную дырку), асимптотически приближаясь к периметру (горизонту) данной дырки. На рис. 2 приведен пример римановой поверхности рода нуль с двумя $\mathbb{Z}_{2}$-орбифолдными точками, одной $\mathbb{Z}_{3}$-орбифолдной точкой и одной дыркой.

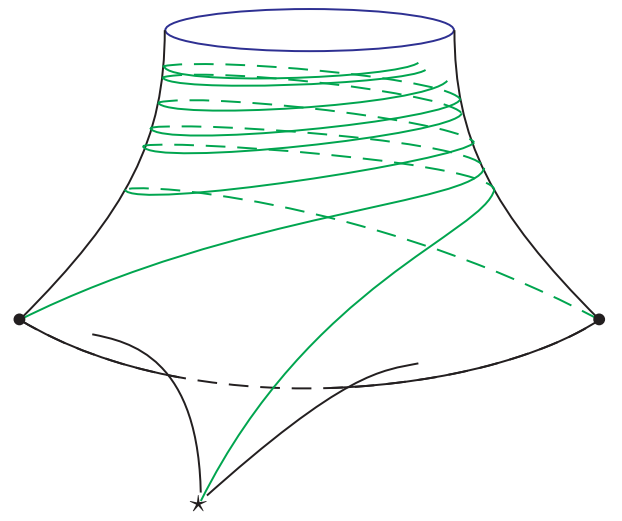

Рис. 2. Пример регулярной римановой поверхности рода нуль с двумя $\mathbb{Z}_{2}$-орбифолдными точками, одной $\mathbb{Z}_{3}$-орбифолдной точкой и одной дыркой. Геодезические линии разбиения начинаются в соответствующих орбифолдных точках и асимптотически навиваются на замкнутую геодезическую, являющуюся периметром дырки.

Рассмотрев прообразы навивающихся на периметр дырки геодезических линий, начинающихся в орбифолдных точках на римановой поверхности, получим, что для $\mathbb{Z}_{2}$-орбифолдной точки прообраз такой геодезической на диске Пуанкаре состоит из двух полубесконечных геодезических, начинающихся в данной точке и противоположно направленных. Можно представить эти две геодезические как одну бесконечную геодезическую, проходящую через прообраз орбифолдной точки. Для $\mathbb{Z}_{3}$-орбифолдной точки эти две полубесконечные геодезические образуют угол $2 \pi / 3$. Их можно при этом рассматривать как две медианы идеального треугольника, центр которого расположен в $\mathbb{Z}_{3}$-орбифолдной точке, и мы достраиваем соответствующий идеальный треугольник как показано на рис. 3 (все вписанные окружности с центрами в $\mathbb{Z}_{3}$-орбифолдных точках имеют один и тот же геодезический радиус $(1 / 2) \log 3)$.

В результате получим картину идеальных треугольников подобно изображенной на рис. 3: фундаментальная область римановой поверхности с отрезанными гиперболическими частями (части поверхности, находящиеся под периметрами дырок) задается объединением идеалъных треуголъников или (при наличии $\mathbb{Z}_{3}$-орбифолдных точек) их третей. Геодезические линии, ограничивающие эту фундаментальную область, представляют собой или попарно отож- 


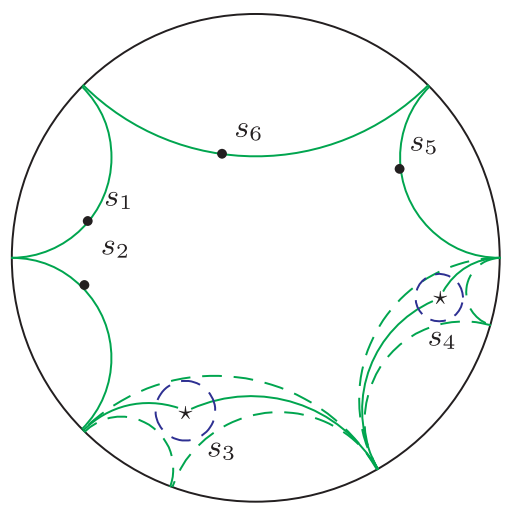

Рис. 3. Диск Пуанкаре с $n=6$ прообразами $s_{i}$ орбифолдных точек (четыре $\mathbb{Z}_{2}$-орбифолдные точки, отмеченные $\bullet$, и две $\mathbb{Z}_{3}$-орбифолдные точки, отмеченные $\star$ ). Сплошные линии обозначают границу фундаментальной области, а пунктирные линии образуют стороны соответствующих идеальных треугольников. Все вписанные окружности с центрами в $\mathbb{Z}_{3}$-орбифолдных точках имеют один и тот же радиус $(1 / 2) \log 3$.

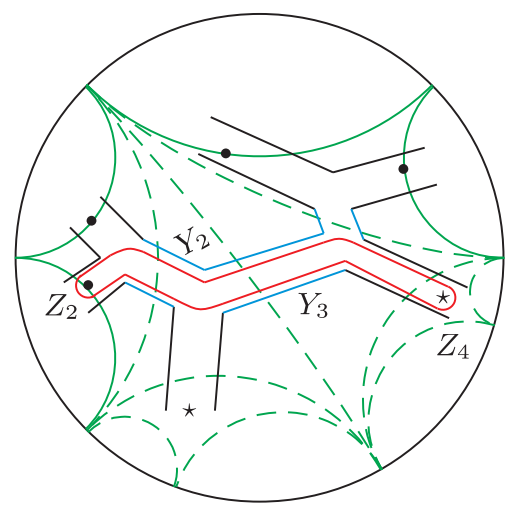

Рис. 4. Диск Пуанкаре с четырьмя $\mathbb{Z}_{2}$-орбифолдными точками, отмеченными $\bullet$, и двумя $\mathbb{Z}_{3}$-орбифолдными точками, отмеченными $\star$, с ассоциированным ленточным графом, дуальным разбиению на идеальные треугольники (дополнительные геодезические линии разбиения на идеальные треугольники внутри фундаментальной области обозначены пунктирными бесконечными линиями). С висячими ребрами ассоциированы вещественные числа $Z_{i}, i=1, \ldots, 6$, а с внутренними ребрами вещественные числа $Y_{2}, Y_{3}$ и $Y_{4}$. Отмечены те из этих чисел, которые дают вклад в замкнутую кривую на графе, отвечающую элементу $\operatorname{Tr} F_{2} Q_{4}$.

дествленные бесконечные геодезические или полубесконечные линии, отождествляемые попарно: две кривые из одной пары начинаются в одной и той же орбифолдной точке. На абсолюте конец каждой линии асимптотически приближается к концу другой линии, образуя тем самым "вершину" идеального 
треугольника. Из этой картины немедленно следует, что все линии, начинающиеся в прообразах орбифолдных точек, должны лежать на границе фундаментальной области.

Построив картину разбиения фундаментальной области с разбиением ее на идеальные треугольники с вершинами на абсолюте на рис. 3, зададим соответствующий этой картине дуальный ленточный граф, представленный на рис. 4 (см. [16], [20], [4]). При этом получим остовный граф с $|\delta|$ новыми висячими вершинами, направленными вовне фундаментальной области и проходящими через прообразы $\mathbb{Z}_{2}$ и $\mathbb{Z}_{3}$-орбифолдных точек. Соответствующая фуксова группа при этом параметризуется вещественными числами $Z_{\alpha}$, присвоенными всем ребрам построенного таким образом ленточного графа.

ПримеР 2.5. Для замкнутого пути, представленного на рис. 4, имеем:

$$
G_{24}=\operatorname{Tr} L X_{Y_{2}} R X_{Y_{3}} L X_{Z_{4}} R X_{Z_{4}} R X_{Y_{3}} L X_{Y_{2}} R X_{Z_{2}} F X_{Z_{2}} .
$$

Поставим теперь в соответствие диску Пуанкаре с $n$ орбифолдными точками древесный ленточный граф с $n$ висячими вершинами. Результат представлен на рис. 4.

\section{3. Преобразования группы классов отображений}

3.1. Пуассонова структура. Одно из наиболее привлекательных свойств техники ленточных графов - очень простая пуассонова структура на пространстве параметров $Z_{\alpha}$. Следующая теорема была сформулирована для поверхностей в отсутствие орбифолдных точек в работе [16] и была обобщена на произвольные графы с висячими вершинами в работе [19] (см. также [12]).

Теорема 3.1. В терминах координат $Z_{\alpha}$ на произвольном биксированном остовном графе, отвечающем поверхности с орбифолдными точками, пуассонова скобка (скобка Вейля-Петерсона) имеет вид

$$
\{f(\mathbf{Z}), g(\mathbf{Z})\}=\sum_{\substack{\text { 3-валентные } \\ \text { вершиньы } \alpha=1}}^{4 g+2 s+|\delta|-4} \sum_{i=1}^{3(\bmod 3)}\left(\frac{\partial f}{\partial Z_{\alpha_{i}}} \frac{\partial g}{\partial Z_{\alpha_{i+1}}}-\frac{\partial g}{\partial Z_{\alpha_{i}}} \frac{\partial f}{\partial Z_{\alpha_{i+1}}}\right),
$$

в котором суммирование идет по всем трехвалентым вершинам графа, а индекс $\alpha_{i}$ нумерует ииклически упорлдоченные (по часовой стрелке, $\alpha_{4} \equiv \alpha_{1}$ ) ребра, входящие в вершину с номером $\alpha$ вне зависимости от того, являются ли эти ребра внутренними или висячими ребрами графа. Эта скобка индуциpyem скобку Голдмана на множестве функиий длин геодезических [14].

Центр этой алгебры Пуассона описывается следующим предложением.

ПреДЛОЖЕНИЕ 3.2. Центр алгебры Пуассона (7) порождается элементами вида $\sum Z_{\alpha}$, где сумма берется по всем ребрам графа (с учетом кратностей) $\Gamma_{g, s, \delta}$, принадлежащим данной граничной компоненте. Это означает, в частности, что каждое висячее ребро, вне зависимости от того, какой орбифолдной точке, $\mathbb{Z}_{2-}$ или $\mathbb{Z}_{3-}$, оно отвечает, входит в такую сумму дважды. Размерность центра, очевидно, равна s. 
3.2. Морфизмы флипов на ленточных графах. В этом пункте приводится полный список преобразований группы классов отображений, которые позволяют изменять числа $\left|\delta_{k}\right|$ орбифолдных точек, связанных с дыркой с номером $k$, изменять циклический порядок орбифолдных точек внутри каждого из подмножеств $\delta_{k}$, производить флип на произвольном внутреннем ребре и, наконец, изменять направление асимптотического накручивания геодезических на периметр дырки (в случае, когда имеется более одной дырки) ${ }^{3}$. Таким образом можно установить морфизм между любыми двумя графами, принадлежащими одному и тому же классу $\Gamma_{g, s,\left|\delta^{(1)}\right|,\left|\delta^{(2)}\right|}$.

3.2.1. Движения Уайтхеда на внутренних ребрах. Координаты $Z_{\alpha}$ представляют собой логарифмы четверных отношений; в случае поверхностей с проколами они являются (тёрстоновскими) координатами сдвига [21], [22].

При заданной нумерации ребер остовного графа Г для поверхности $\Sigma$ и в предположении, что ребро $\alpha$ оканчивается в разных вершинах, из остовного графа $\Gamma$ можно получить другой остовный граф $\Gamma_{\alpha}$ поверхности $\Sigma$, если мы сожмем, а потом растянем ребро $\alpha$ (ребро с переменной $Z$ на рис. 5 ), получив тем
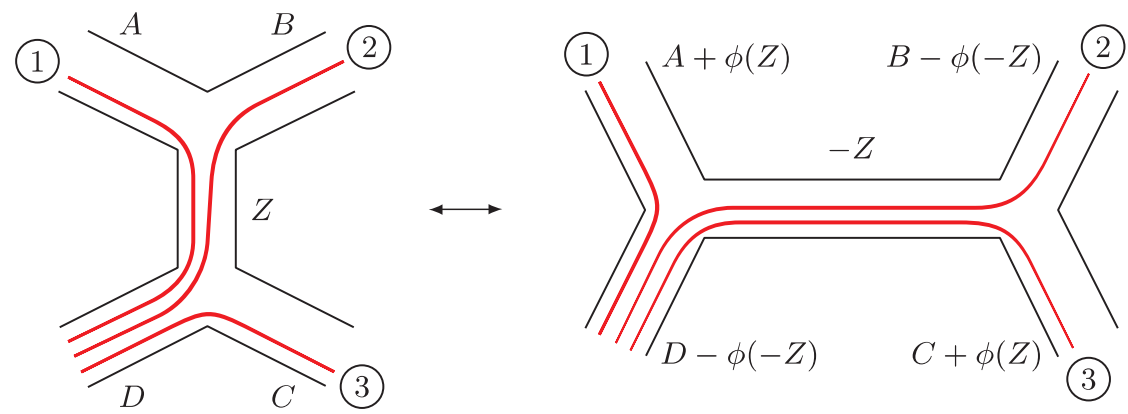

Рис. 5. Флип, или движение Уайтхеда, на множестве координат сдвига $Z_{\alpha}$. Внешние ребра могут быть висячими, но ребро, относительно которого осуществляется морфизм, должно быть внутренним ребром. Совпадающими числами в кружках отмечены геодезические линии, которые отождествляются в левой и правой частях рисунка.

самым новый остовный граф $\Gamma_{\alpha}$, как показано на рисунке. Далее, маркировка ребер $Г$ задает естественным образом маркировку ребер графа $\Gamma_{\alpha}$, в которой вертикальное ребро с индексом $Z$ на рис. 5 отвечает горизонтальному ребру с индексом $-Z$. Будем говорить, что граф $\Gamma_{\alpha}$ получается из графа $\Gamma$ с помощью движения Уайтхеда (или флипа) относительно ребра с индексом $\alpha$. Маркировка ребер остовного графа Г приводит к естественной маркировке на множестве ребер остовного графа $\Gamma_{\alpha}$; таким образом можно устанавливать морфизм между двумя остовными графами $Г$ и $\Gamma_{\alpha}$.

\footnotetext{
${ }^{3}$ На языке кластерных алгебр [23], [24], [19] это означает возможность производить мутацию на произволъном ребре любого ленточного графа, возможно с висячими ребрами или с внутренними ребрами, концы которых входят в одну и ту же вершину.
} 
ПРЕДЛОЖЕНИЕ 3.3 [25], [26]. Полагая $\phi(Z)=\log \left(1+e^{Z}\right)$, получим, что в обозначениях рис. 5 для координат сдвигов на прилегающих ребрах действие движения Уайтхеда имеет вид:

$$
W_{Z}:(A, B, C, D, Z) \rightarrow(A+\phi(Z), B-\phi(-Z), C+\phi(Z), D-\phi(-Z),-Z)
$$

В различных частных случаях, когда прилегающие ребра отождествляются, применяя одни и те же обозначения для ребер и координат, с ними связанных, получим: если $A=C$, то $A^{\prime}=A+2 \phi(Z)$; если $B=D$, mо $B^{\prime}=B-2 \phi(-Z)$; если $A=B($ или $C=D)$, то $A^{\prime}=A+Z\left(\right.$ или $\left.C^{\prime}=C+Z\right) ;$ если $A=D($ или $B=C)$, то $A^{\prime}=A+Z\left(\right.$ или $\left.B^{\prime}=B+Z\right)$. Любое подмножество ребер $A, B, C$ и $D$ может бъть висячими ребрами графа.

Имеют также место две простые, но важные леммы, устанавливающие свойства инвариантности относительно морфизма флипа [25], [26].

ЛЕмма 3.4. Преобразование (8) сохраняет следы произведений (4).

ЛЕмма 3.5. Преобразование (8) сохраняет пуассонову структуру (7) на множестве координат сдвигов.

3.2.2. Движения Уайтхеда на висячих ребрах. Выбор других представителей орбифолдных точек на диске Пуанкаре приводит к выбору другой фундаментальной области с измененным циклическим порядком на множестве (прообразов) орбифолдных точек $s_{i}\left(i=1, \ldots,\left|\delta_{k}\right|\right)$.

В случае орбифолдных римановых поверхностей имеется возможность перемещать орбифолдные точки из одного подмножества $\delta_{k}$ в другое (при этом меняются числа $\left.\left|\delta_{k}\right|\right)$ и изменять циклическое упорядочение орбифолдных точек внутри подмножеств $\delta_{k}$. Эти симметрии обеспечиваются следующими двумя морфизмами, отвечающими в определенной мере преобразованиям флипа на висячих ребрах.

Преобразование флипа на ребре с $\mathbb{Z}_{2}$-орбифолдной точкой. На рис. 6 показано, как меняется фундаментальная область при замене прообраза $s_{\alpha_{i}}$

на другой прообраз $s_{\alpha_{i}}^{\prime}$ той же самой орбифолдной точки, получаемый под действием группового элемента $F_{i+1}$, т. е. при повороте на угол $\pi$ вокруг точки $s_{\alpha_{i+1}}$. В результате изменяется циклический порядок: вместо $\left\{\ldots, s_{\alpha_{i}}\right.$, $\left.s_{\alpha_{i+1}}, \ldots\right\}$ получаем $\left\{\ldots, s_{\alpha_{i+1}}, s_{\alpha_{i}}^{\prime}, \ldots\right\}$.

Лемма 3.6. Преобразование на рис. 7 с

$$
\left\{\widetilde{Y}_{1}, \widetilde{Y}_{2}, \widetilde{Z}\right\}=\left\{Y_{1}-\log \left(1+e^{-2 Z}\right), Y_{2}+\log \left(1+e^{2 Z}\right),-Z\right\}
$$

представляет собой морбизм графов. Такой морфизм сохраняет как пуассонову структуру (7), так и геодезические функиии. На рис. 7 любое из ребер с Y-переменными (или оба этих ребра) могут быть висячими ребрами (бормула преобразования при этом не меняется). 

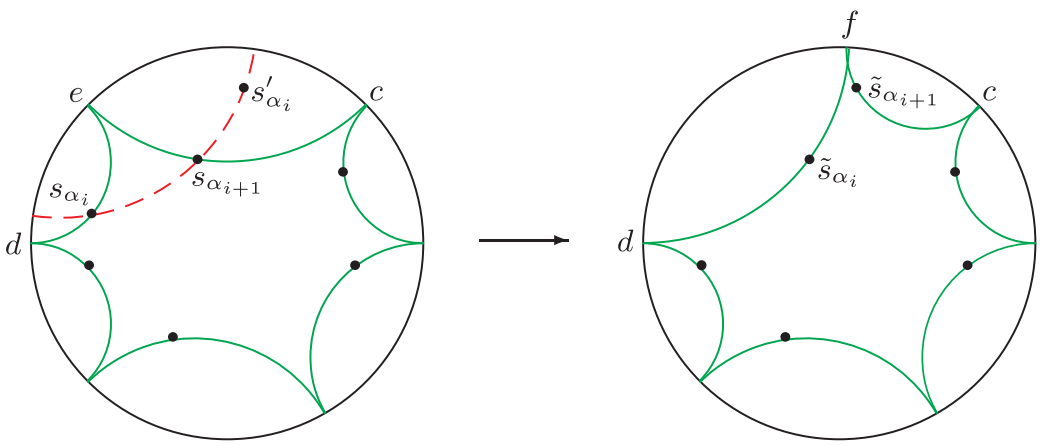

Рис. 6. Замена прообраза $s_{\alpha_{i}}$ орбифолдной точки на другой прообраз $s_{\alpha_{i}}^{\prime}$, полученный с помощью поворота на угол $\pi$ вокруг прообраза $s_{\alpha_{i+1}}$ соседней $\mathbb{Z}_{2}$-орбифолдной точки. Две граничные кривые заменяются двумя новыми граничными кривыми, в то время как остальная часть фундаментальной области не меняется. Циклический порядок на множестве $\left\{s_{k}\right\}$ также меняется, что отвечает преобразованию флипа на висячем ребре.
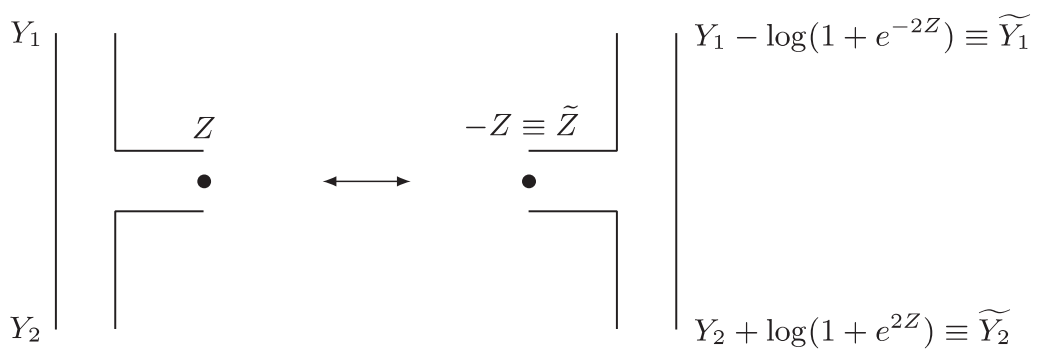

Рис. 7. Флип, или движение Уайтхеда, на множестве координат сдвигов при преобразовании висячего ребра $Z$, отвечающего $\mathbb{Z}_{2}$-орбифолдной точке (отмеченной жирной точкой). Любое из ребер $Y_{1}$ и $Y_{2}$ (или оба ребра) могут быть висячими ребрами.

ДокАЗАТЕЛЬСтво. Проверка сохранения пуассоновых соотношений (7) производится довольно просто, а для следов по путям имеются четыре случая, в каждом из которых выполняются следующие четыре $(2 \times 2)$-матричные равенства:

$$
\begin{aligned}
X_{Y_{2}} L X_{Z} F X_{Z} L X_{Y_{1}} & =X_{\widetilde{Y}_{2}} L X_{\widetilde{Y}_{1}}, \\
X_{Y_{1}} R X_{Z} F X_{Z} L X_{Y_{1}} & =X_{\widetilde{Y}_{1}} L X_{\widetilde{Z}} F X_{\widetilde{Z}^{2}} R X_{\widetilde{Y}_{1}}, \\
X_{Y_{2}} R X_{Y_{1}} & =X_{\widetilde{Y}_{2}} R X_{\widetilde{Z}} F X_{\widetilde{Z}} R X_{\widetilde{Y}_{1}}, \\
X_{Y_{2}} L X_{Z} F X_{Z} R X_{Y_{2}} & =X_{\widetilde{Y}_{2}} R X_{\widetilde{Z}} F X_{\widetilde{Z}} L X_{\widetilde{Y}_{2}} .
\end{aligned}
$$

Преобразование флипа на ребре $с \mathbb{Z}_{3}$-орбифолдной точкой. На рис. 8 представлено изменение фундаментальной области при вращении части кривых, ограничивающих ее, вокруг прообраза $\mathbb{Z}_{3}$-орбифолдной точки. При этом полу- 

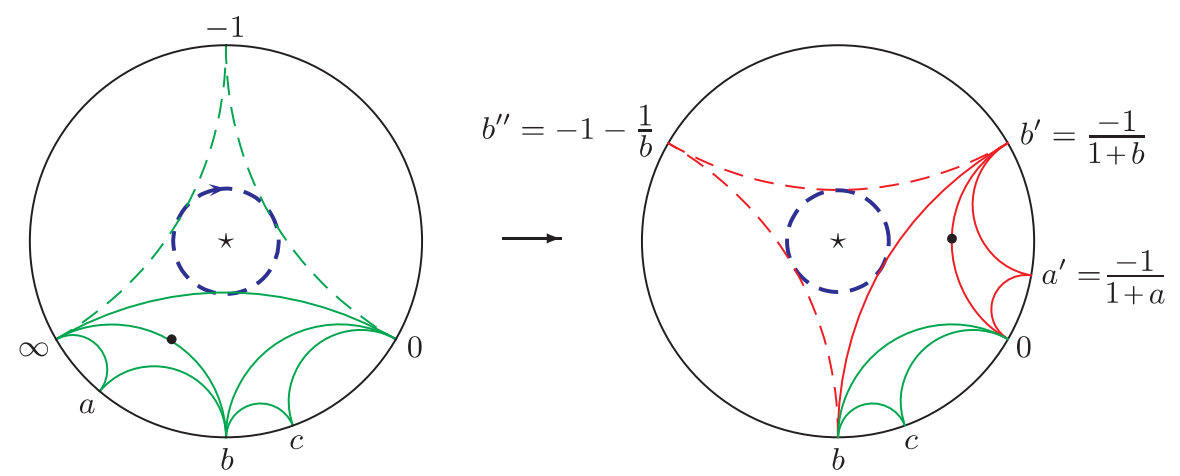

Рис. 8. Вращение части разбиения (ограниченной кривой между точками $b$ и $\infty)$ вокруг прообраза $\mathbb{Z}_{3}$-орбифолдной точки в центре; соответствующее изменение ленточного графа представлено на рис. 9.

чаются две новые точки $b^{\prime}$ и $a^{\prime}$ на абсолюте вместе с соответствующими четверными отношениями. (Остальные граничные кривые не меняются.) Вычисляя эти четверные отношения, приходим к следующему закону преобразования.

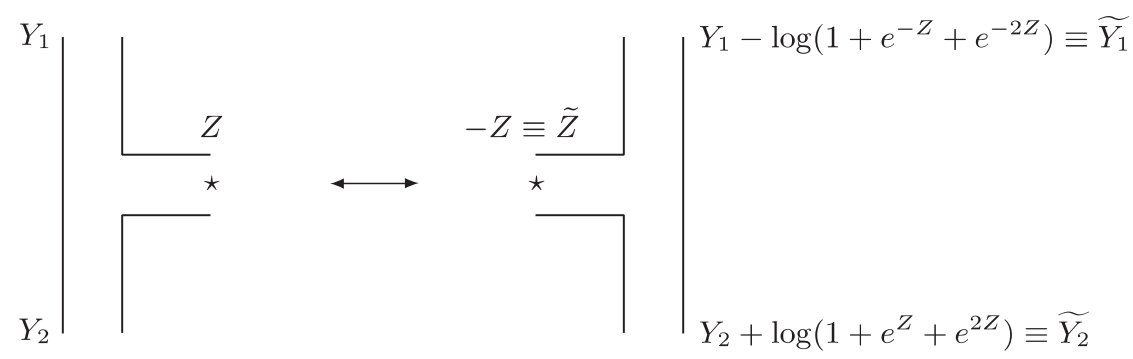

Рис. 9. Флип, или движение Уайтхеда, на множестве координат сдвигов при преобразовании висячего ребра $Z$, отвечающего $\mathbb{Z}_{3}$-орбифолдной точке (отмеченной звездой). Любое из ребер $Y_{1}$ и $Y_{2}$ (или оба ребра) могут быть висячими ребрами

Лемма 3.7. Преобразование на рис. 9 с

$$
\left\{\widetilde{Y}_{1}, \widetilde{Y}_{2}, \widetilde{Z}\right\}=\left\{Y_{1}-\log \left(1+e^{-Z}+e^{-2 Z}\right), Y_{2}+\log \left(1+e^{Z}+e^{2 Z}\right),-Z\right\}
$$

представляет собой морфизм графов. Такой морфизм сохраняет как пуассонову структуру (7), так и геодезические функиии. На рис. 9 любое из ребер с Y-переменными (или оба этих ребра) могут быть висячими ребрами.

ДокАЗАТЕЛЬство. Проверка сохранения пуассоновых соотношений (7) снова производится довольно просто, а для следов по путям имеются четыре слу- 
чая, в каждом из которых выполняются следующие $(2 \times 2)$-матричные равенства:

$$
\begin{aligned}
X_{Y_{2}} L X_{Z} L X_{Z} L X_{Y_{1}} & =X_{\widetilde{Y}_{2}} L X_{\widetilde{Y}_{1}}, \\
X_{Y_{1}} R X_{Z} L X_{Z} L X_{Y_{1}} & =X_{\widetilde{Y}_{1}} L X_{\widetilde{Z}} L X_{\widetilde{Z}} R X_{\widetilde{Y}_{1}}, \\
X_{Y_{2}} R X_{Y_{1}} & =X_{\widetilde{Y}_{2}} R X_{\widetilde{Z}} R X_{\widetilde{Z}} R X_{\widetilde{Y}_{1}}, \\
X_{Y_{2}} L X_{Z} R X_{Z} R X_{Y_{2}} & =X_{\widetilde{Y}_{2}} R X_{\widetilde{Z}} R X_{\widetilde{Z}} L X_{\widetilde{Y}_{2}} .
\end{aligned}
$$

Стандартным образом в случае, когда после последовательности морфизмов получается граф того же комбинаторного типа, что и исходный (без учета нумерации ребер, но с учетом двух разных типов орбифолдных вершин), такому морфизму можно поставить в соответствие преобразование из группы классов отображений, перейдя таким образом от группоида морфизмов к группе модулярных преобразований.

3.2.3. Изменение направления намотки. Последнее преобразование группы классов отображений изменяет знак периметра дырки:
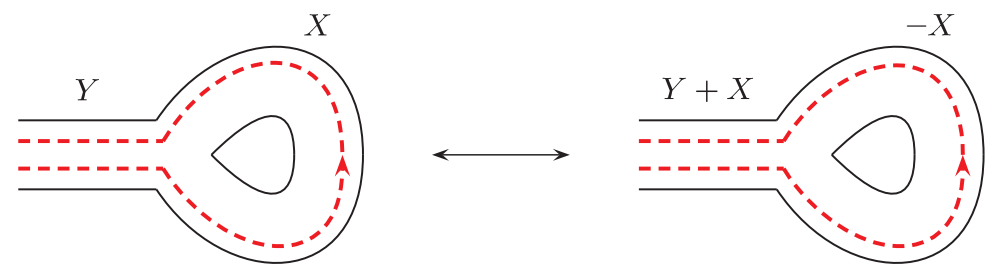

То, что это преобразование сохраняет геодезические функции, следует из двух матричных равенств:

$$
\begin{aligned}
& X_{Y} L X_{X} L X_{Y}=X_{Y+X} L X_{-X} L X_{Y+X}, \\
& X_{Y} R X_{X} R X_{Y}=X_{Y+X} R X_{-X} R X_{Y+X},
\end{aligned}
$$

и мы, тем самым, можем расширить группу классов отображений декорированного пространства Тейхмюллера $\mathscr{T}_{g, s, \delta}^{H}$, добавив симметрии между листами $2^{s}$-разветвленного накрытия "подлинного" (недекорированного) пространства Тейхмюллера $\mathscr{T}_{g, s, \delta}$. (Напомним, что процедура декорирования состоит в присваивании каждой из дырок соответствующего знака: положительного или отрицательного в зависимости от направления намотки геодезических кривых разбиения на периметр дырки.)

Геометрический смысл этого преобразования понятен: мы изменяем направление намотки на периметр дырки для всех кривых разбиения на идеальные треугольники, которые асимптотически наматываются на данную дырку, как показано на рис. 2. 


\section{4. Алгебры геодезических функций}

4.1. Структура геодезических и ламинаций на ленточных графах. Каждому замкнутому пути на ленточном графе $\Gamma_{g, s,|\delta|}$, представляющем собой остовный граф римановой поверхности рода $g$ с $s$ дырками и $|\delta| \mathbb{Z}_{2^{-}}$и $\mathbb{Z}_{3}$-орбифолдными точками, поставим в соответствие замкнутый путь на римановой поверхности. Этому пути в свою очередь отвечает замкнутая геодезическая, представляющая собой образ инвариантной оси соответствующего гиперболического элемента фуксовой группы.

ОПРЕДЕЛЕНИЕ 4.1. Геодезическая ламинация (ГЛ) (или множественная кривая) на орбифолдной римановой поверхности $\Sigma_{g, s,|\delta|}$ есть набор не(само)пересекающихся геодезических кривых (с кратностями), включая кривые, которые заканчиваются в орбифолдных точках (это возможно, только если данная кривая соединяет две $\mathbb{Z}_{2}$-орбифолдные точки). В последнем случае только одна кривая (с кратностью), заканчивающаяся в данной $\mathbb{Z}_{2}$-орбифолдной точке $s_{i}$, может входить в ГЛ.

Алгебраический аналог ГЛ есть GM-функция (GM - сокращение от Geodesic Multicurve):

$$
\mathrm{GM}:=\prod_{\gamma \in \Gamma Л} G_{\gamma}^{m_{\gamma}},
$$

где произведение берется по всем геодезическим $\gamma$, входящим в ГЛ с кратностями $m_{\gamma}$.

Напомним важное соотношение, справедливое в $\mathbb{P S L}(2)$ :

$$
\operatorname{Tr} \gamma_{A} \operatorname{Tr} \gamma_{B}=\operatorname{Tr}\left(\gamma_{A} \gamma_{B}\right)+\operatorname{Tr}\left(\gamma_{A} \gamma_{B}^{-1}\right)
$$

Это соотношение позволяет распутывать пересечения двух геодезических кривых $A$ и $B$, как показано на рис. 10, и его поэтому логично назвать pacnymbваюшим соотношением.
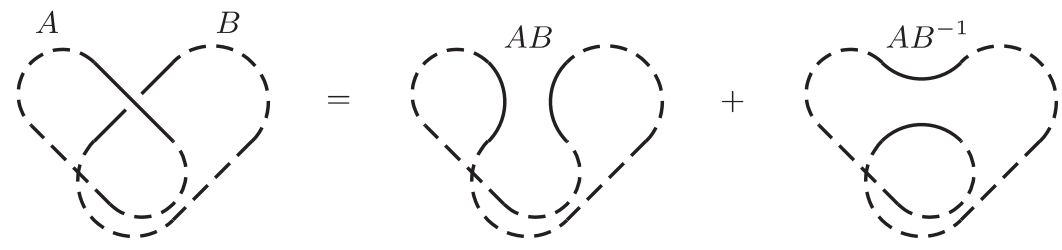

Рис. 10. Классическое распутывающее соотношение

Для геодезических, встречающихся в орбифолдной точке, справедливо распутывающее соотношение, представленное на рис. 11.

4.2. Пуассоновы скобки для геодезических функций. Алгебры функций длин геодезических были построены в работах [25], [26] на основе постулированных пуассоновых соотношений (7) на уровне координат сдвигов $Z_{\alpha}$ пространств Тейхмюллера. 


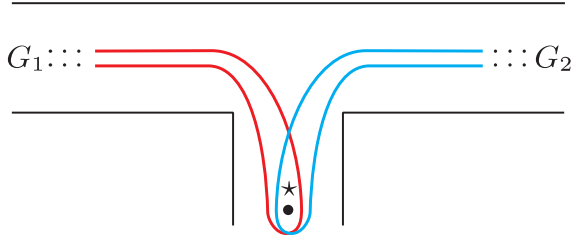

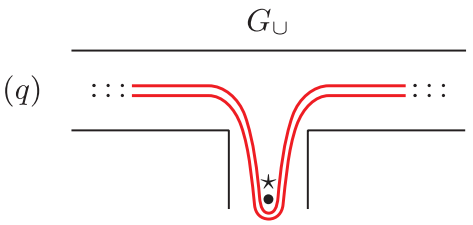

(a)

(1)

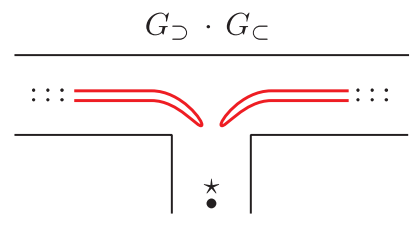

(c)

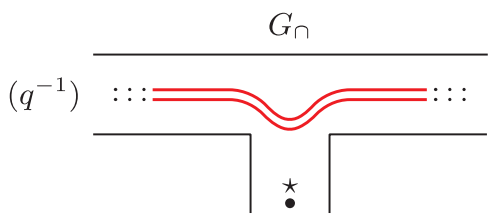

(b)

0

(0)

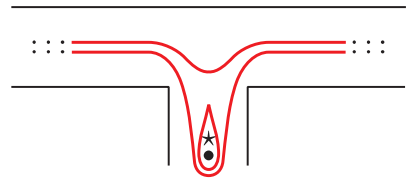

(d)

Рис. 11. Пример двух геодезических кривых, пересекающихся в орбифолдной точке, отмеченной жирной точкой или звездой. Имеются четыре гомотопических типа разрешения двух пересечений кривых в этой картине, представленные случаями (a)-(d). Ламинация в случае (d) содержит петлю, обходящую только вокруг орбифолдной точки. Для точечных вершин эта петля даст $\operatorname{Tr} F=0$, так что вся GM-функция в этом случае зануляется. Для звездчатых вершин соответствующая петля дает $|\operatorname{Tr} L|=\operatorname{Tr} R=1$. Множители в скобках отвечают случаю квантовых распутывающих соотношений и представляют собой веса, с которыми соответствующие (квантовые) (Q)GM входят в выражения для произведения $G_{1}^{\hbar} G_{2}^{\hbar}$ квантовых геодезических функций.

Отметим сначала, что две геодезические функции коммутируют, если соответствующие геодезические кривые вкладываются без пересечений в остовный

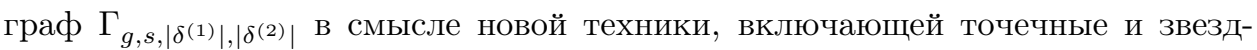
чатые вершины. В силу выполнения условия Лейбница для скобки Пуассона достаточно рассмотреть одни лишь "простые" пересечения пары геодезических кривых, когда соответствующие геодезические функции $G_{\gamma_{1}}$ и $G_{\gamma_{2}}$ имеют вид:

$$
\begin{aligned}
G_{\gamma_{1}} & =\operatorname{Tr} \ldots X_{C} R X_{Z} L X_{A} \ldots, \\
G_{\gamma_{2}} & =\operatorname{Tr} \ldots X_{B} L X_{Z} R X_{D} \ldots .
\end{aligned}
$$

Положение ребер $A, B, C, D$ и $Z$ предполагается таким же, как и на рис. 5 . Многоточия в формулах $(13),(14)$ обозначают произвольные произведения матриц $R, L, X_{Z_{i}}$ и $F$; функции $G_{\gamma_{1}}$ и $G_{\gamma_{2}}$ должны отвечать замкнутым геодезическим, но в остальном они произвольны. 
Прямые вычисления дают ответ:

$$
\left\{G_{\gamma_{1}}, G_{\gamma_{2}}\right\}=\frac{1}{2}\left(G_{\gamma_{1} \gamma_{2}^{-1}}-G_{\gamma_{1} \gamma_{2}}\right)
$$

(ср. (12)). Эти соотношения были впервые получены У. М. Голдманом [14] в непрерывной параметризации (классическая алгебра Тураева-Виро).

В силу леммы 3.6 пуассоновы соотношения полностью определяются гомотопическими типами кривых на римановой поверхности $\Sigma_{g, s, \delta}$ и не зависят от выбора остовного графа. В частности, все орбифолдные точки можно всегда разместить на одной и той же компоненте границы.

Для двух кривых $\gamma_{1}$ и $\gamma_{2}$ с произвольным числом пересечений скобка Пуассона находится с применением следующих правил:

- берется сумма произведений геодезических функций не(само)пересекающихся кривых (GM), получающихся при применении пуассоновых соотношений (15) в одной из точек пересечения и классических соотношений распутывания (12) во всех остальных точках пересечения с последующим взятием суммы по всем возможным случаям;

- если в процессе вычислений встречается пустая (стягиваемая) петля, то ей следует сопоставить множитель -2; это присваивание [5] обеспечивает выполнение движений Рейдемейстера на множестве геодезических кривых, в силу чего скобки Пуассона зависят только от гомотопического класса вложения кривой на поверхность;

- если в процессе вычислений получается кривая, гомеоморфная обходу вокруг точечной вершины, то этой кривой отвечает множитель $\operatorname{Tr} F=0$, и вся соответствующая GM зануляется; если получается кривая, гомеоморфная обходу вокруг звездчатой вершины, то соответствующая геодезическая функция в GM полагается равной $\operatorname{Tr} R=|\operatorname{Tr} L|=1$.

Все эти правила применимы, например, к пуассоновой скобке геодезических функций, отвечающих геодезическим, изображенным на рис. 11. В обозначениях этого рисунка имеем, что $\left\{G_{1}, G_{2}\right\}=G_{\cup}-G_{\cap}$ как в случае точечной, так и в случае звездчатой вершины.

\section{5. Специальные случаи геодезических алгебр}

5.1. Алгебра $A_{n}$. Одним из простейших примеров орбифолдных римановых поверхностей оказывается диск Пуанкаре с $n \geqslant 3 \mathbb{Z}_{2}$-орбифолдными точками на диске; соответствующая риманова поверхность есть $\Sigma_{0,1, n, 0}$. В этом случае ленточный граф $\Gamma_{0,1, n, 0}$ представляет собой древесный граф типа представленного на рис. 12 для случаев $n=3,4, \ldots$. Занумеруем $n$ точечных вершин против часовой стрелки, $i, j=1, \ldots, n$, и рассмотрим алгебру специального подмножества геодезических функций ${ }^{4}$.

5.1.1. Пуассоновы соотношения для алгебры $A_{n}$. Обозначим через $G_{i, j}$ с $i<j$ геодезические функции, отвечающие геодезическим, обходящим вокруг ровно двух точечных вершин с индексами $i$ и $j$. Примеры таких путей для

\footnotetext{
${ }^{4}$ В терминологии кластерных алгебр (см. [27]) эти алгебры обозначались $A_{n-2}$.
} 
$n=3$ и $n=4$ показаны на рис. 12 . Соответствующие геодезические функции равны $\operatorname{Tr} F_{i} F_{j}$, а соответствующие геодезические проходят дважды по пути, соединяющему соответствующие орбифолдные точки $s_{i}$ и $s_{j}$ и испытывающему отражения в этих двух орбифолдных точках.
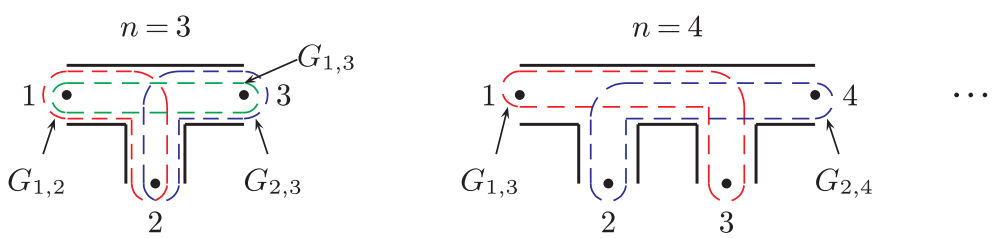

Рис. 12. Остовные графы для алгебр $A_{n}$ при $n=3,4, \ldots$. Отмечены примеры геодезических, геодезические функции $G_{i, j}$ которых входят в базис соответствующих алгебр.

Выразив с помощью распутывающего соотношения (12) зависимость от $\operatorname{Tr} \gamma_{A} \gamma_{B}$ в пуассоновых соотношениях (15), пуассонову алгебру можно замкнуть на уровне набора геодезических функций $G_{i, j}, 1 \leqslant i<j \leqslant n$, образующего конечное подмножество:

$$
\begin{aligned}
& \left\{G_{i, k}, G_{j, l}\right\}=0 \quad \text { при } i<k<j<l \text { и при } i<j<l<k \text {, } \\
& \left\{G_{i, k}, G_{j, l}\right\}=2\left(G_{i, j} G_{k, l}-G_{i, l} G_{k, j}\right) \quad \text { при } i<j<k<l \text {, } \\
& \left\{G_{i, k}, G_{k, l}\right\}=G_{i, k} G_{k, l}-2 G_{i, l} \quad \text { при } i<k<l \text {, } \\
& \left\{G_{i, k}, G_{j, k}\right\}=-\left(G_{i, k} G_{j, k}-2 G_{i, j}\right) \quad \text { при } i<j<k \text {, } \\
& \left\{G_{i, k}, G_{i, l}\right\}=-\left(G_{i, k} G_{i, l}-2 G_{k, l}\right) \quad \text { при } i<k<l \text {. }
\end{aligned}
$$

Рассмотрим базис $\gamma_{1}, \ldots, \gamma_{n}$ в фуксовой группе $\Delta_{0,1, n, 0}$,

$$
-\operatorname{Tr}\left(\gamma_{i} \gamma_{j}\right)=G_{i, j}
$$

так что $G_{i, j}=2 \cosh \left(\ell_{i, j} / 2\right) \geqslant 2$, где через $\ell_{i, j}$ обозначены длины замкнутых геодезических. Матрица

$$
\gamma_{\infty}:=\left(\gamma_{1} \gamma_{2} \ldots \gamma_{n}\right)^{-1}
$$

имеет собственные значения $(-1)^{n-1} e^{ \pm P / 2}$, где $P-$ взятая со знаком длина периметра дырки:

$$
P=2 \sum_{\alpha=1}^{2 n-3} Z_{\alpha}
$$

(сумма берется по всем ребрам графа).

5.1.2. Действие группы классов отображений на $A_{n}$. Преобразования из группы кос переставляют между собой орбифолдные точки с помощью вращений $i$-й точки вокруг точки с номером $i+1$, как показано на рис. 6 . 
Действие группы классов отображений для алгебры $A_{n}$ задается соответствующим действием группы кос [8], [12]: построим верхнетреугольную матрицу

$$
\mathscr{A}=\left(\begin{array}{ccccc}
1 & G_{1,2} & G_{1,3} & \ldots & G_{1, n} \\
0 & 1 & G_{2,3} & \ldots & G_{2, n} \\
0 & 0 & 1 & \ddots & \vdots \\
\vdots & \vdots & \ddots & \ddots & G_{n-1, n} \\
0 & 0 & \ldots & 0 & 1
\end{array}\right)
$$

отождествив ее элементы $G_{i, j}$ с геодезическими функциями. Действие порождающего элемента из группы кос $\beta_{i, i+1}$ задается формулами

$$
\beta_{i, i+1} \mathscr{A}=\widetilde{\mathscr{A}}, \quad \text { где } \begin{cases}\widetilde{G}_{i+1, j}=G_{i, j}, & j>i+1, \\ \widetilde{G}_{j, i+1}=G_{j, i}, & j<i, \\ \widetilde{G}_{i, j}=G_{i, j} G_{i, i+1}-G_{i+1, j}, & j>i+1, \\ \widetilde{G}_{j, i}=G_{j, i} G_{i, i+1}-G_{j, i+1}, & j<i, \\ \widetilde{G}_{i, i+1}=G_{i, i+1} . & \end{cases}
$$

Это преобразование можно представить весьма удобным способом, если ввести специальные матрицы $B_{i, i+1}$ блочно-диагонального вида (см. [11])

$$
\left.B_{i, i+1}=\begin{array}{c}
\vdots \\
i+1 \\
\vdots \\
\end{array}\right)\left(\begin{array}{cccccccc}
1 & & & & & & \\
& \ddots & & & & & \\
& & 1 & & & & \\
& & & G_{i, i+1} & -1 & & \\
& & 1 & 0 & & & \\
& & & & & 1 & & \\
& & & & & & \ddots & \\
& & & & & & 1
\end{array}\right) .
$$

Тогда действие генератора группы кос $\beta_{i, i+1}$ на $\mathscr{A}$ принимает вид матричного произведения:

$$
\beta_{i, i+1} \mathscr{A}=B_{i, i+1} \mathscr{A} B_{i, i+1}^{T}
$$

где $B_{i, i+1}^{T}$ - транспонированная матрица $B_{i, i+1}$. В этом подходе особенно легко доказываются стандартные соотношения группы кос:

$$
\beta_{i-1, i} \beta_{i, i+1} \beta_{i-1, i}=\beta_{i, i+1} \beta_{i-1, i} \beta_{i, i+1}, \quad 2 \leqslant i \leqslant n-1,
$$

а также одно дополнительное соотношение [11]:

$$
\left(\beta_{n-1, n} \beta_{n-2, n-1} \cdots \beta_{2,3} \beta_{1,2}\right)^{n}=\mathbb{E} .
$$

5.2. Алгебра $D_{n}$. Рассмотрим теперь риманову поверхность с двумя дырками (кольцо) и с $n \geqslant 2 \mathbb{Z}_{2}$-орбифолдными точками, $\Sigma_{0,2, n, 0}$. Ленточный граф $\Gamma_{0,2, n}$ с $n=4$ представлен на рис. 13. 

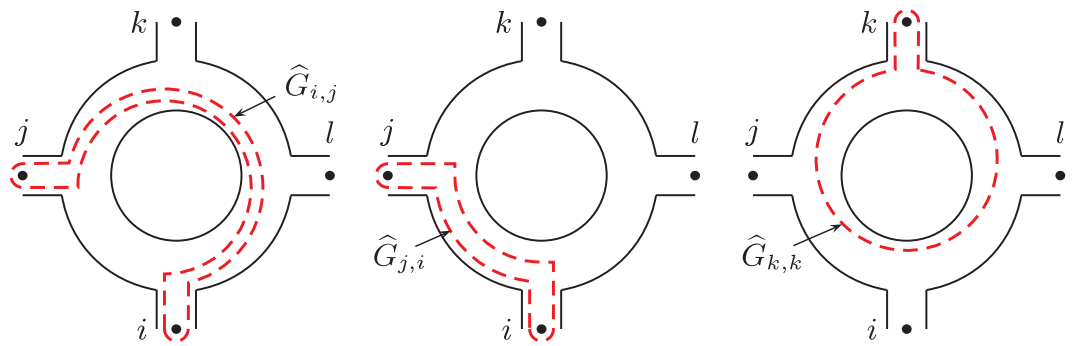

Рис. 13. Типичные геодезические, отвечающие геодезическим функциям, образующим набор генераторов алгебры $D_{n}$ в подходе работы [12]

5.2.1. Пуассоновы соотношения для алгебры $D_{n}$. Алгебры геодезических функций на поверхности $\Sigma_{0,2, n, 0}$ были построены в работе [12]. Для того чтобы замкнуть эту пуассонову алгебру, требуется бо́льшее количество геодезических функций, чем в случае алгебры $A_{n}$, а именно, требуются функции $\widehat{G}_{i, i}$, отвечающие геодезическим, обходящим вокруг точечной вершины с индексом $i$ и дырки, а для каждой (симметризованной) пары различных индексов $i, j=1, \ldots, n$ добавляются две геодезические, обходящие вокруг точечных вершин с индексами $i$ и $j: \widehat{G}_{i, j}$ и $\widehat{G}_{j, i}$. Порядок нижних индексов зависит от того, с какой стороны геодезическая линия обходит дырку (вторую граничную компоненту), см. рис. 13. Из этого рисунка следует, что ленточный граф задает естественное циклическое упорядочение на множестве точечных вершин. Полное число генераторов алгебры $D_{n}$ равно, тем самым, $n^{2}$. Очевидно, что $\widehat{G}_{i, j}$ с $1 \leqslant i<j \leqslant n$ образуют одну (среди $n$ возможных) подалгебр $A_{n}$ в алгебре $D_{n}$.

Скобки Пуассона для алгебр $D_{n}$ содержат члены вплоть до третьего порядка [12], [6]; в нижеприведенных формулах предполагается циклическое упорядочение на множестве индексов $\{i, l, k, j\}$ :

$$
\begin{aligned}
\left\{\widehat{G}_{i, k}, \widehat{G}_{j, l}\right\} & =2\left(\widehat{G}_{j, k} \widehat{G}_{i, l}-\widehat{G}_{j, i} \widehat{G}_{l, k}\right) ; \\
\left\{\widehat{G}_{i, k} \widehat{G}_{k, j}\right\} & =\widehat{G}_{i, k} \widehat{G}_{k, j}-2 \widehat{G}_{i, j} ; \\
\left\{\widehat{G}_{k, i}, \widehat{G}_{j, j}\right\} & =2\left(\widehat{G}_{k, j} \widehat{G}_{i, i}-\widehat{G}_{j, i} \widehat{G}_{k, k}\right) ; \\
\left\{\widehat{G}_{k, j}, \widehat{G}_{k, k}\right\}= & \widehat{G}_{k, j} \widehat{G}_{k, k}-2 \widehat{G}_{j, j}, \quad\left\{\widehat{G}_{j, i}, \widehat{G}_{j, j}\right\}=-\widehat{G}_{j, i} \widehat{G}_{j, j}+2 \widehat{G}_{i, i} ; \\
\left\{\widehat{G}_{i, i}, \widehat{G}_{k, k}\right\}= & \widehat{G}_{i, k}-\widehat{G}_{k, i} ; \\
\left\{\widehat{G}_{l, i}, \widehat{G}_{j, k}\right\}= & 2\left(\widehat{G}_{i, k} \widehat{G}_{l, j}-\widehat{G}_{k, i} \widehat{G}_{j, l}+\widehat{G}_{j, i} \widehat{G}_{k, l}-\widehat{G}_{i, j} \widehat{G}_{l, k}\right) \\
& \quad+\quad 4\left(\widehat{G}_{l, k} \widehat{G}_{i, i} \widehat{G}_{j, j}-\widehat{G}_{j, i} \widehat{G}_{l, l} \widehat{G}_{k, k}\right) ; \\
\left\{\widehat{G}_{l, i}, \widehat{G}_{i, k}\right\}= & \widehat{G}_{l, i} \widehat{G}_{i, k}-2 \widehat{G}_{i, l} \widehat{G}_{k, i}-4 \widehat{G}_{l, l} \widehat{G}_{k, k}+2 \widehat{G}_{k, l}+2 \widehat{G}_{l, k}\left(\widehat{G}_{i, i}\right)^{2} ; \\
\left\{\widehat{G}_{j, l}, \widehat{G}_{l, j}\right\}= & 2\left(\widehat{G}_{l, l}\right)^{2}-2\left(\widehat{G}_{j, j}\right)^{2} .
\end{aligned}
$$

В работе [9] алгебры $D_{n}$ были также получены с помощью редукций из бесконечномерных алгебр $\mathfrak{D}_{n}$, описанных ниже в разделе 7 .

Как и в случае алгебры $A_{n}$, группа классов отображений поверхности $\Sigma_{0,2, n, 0}$ представляет собой группу кос. Но в этом случае к $n-1$ генераторам $\beta_{i, i+1}$ с $i=$ $1, \ldots, n-1$ необходимо добавить новый генератор $\beta_{n, 1}$. Заметим, что ни скобки 
Пуассона, ни действие группы кос не зависят от длины периметра добавленной дырки, так что алгебры $D_{n}$ можно рассматривать как абстрактные пуассоновы алгебры на $n^{2}$ формальных элементах $\widehat{G}_{i, j}, i, j=1, \ldots, n$.

5.2.2. Соотношения группы кос для алгебр $D_{n}$. Действие группы кос на генераторах $\widehat{G}_{i, j}, i, j=1, \ldots, n$, алгебры $D_{n}$ можно явно представить в следующем виде:

$$
\beta_{i, i+1} \widehat{G}_{k, l}=\widetilde{\widehat{G}}_{k, l},: \begin{cases}\widetilde{\widehat{G}}_{i+1, k}=\widehat{G}_{i, k}, & k \neq i, i+1, \\ \widehat{\widehat{G}}_{i, k}=\widehat{G}_{i, k} \widehat{G}_{i, i+1}-\widehat{G}_{i+1, k}, & k \neq i, i+1, \\ \widehat{\widehat{G}}_{k, i+1}=\widehat{G}_{k, i}, & k \neq i, i+1, \\ \widehat{\widehat{G}}_{k, i}=\widehat{G}_{k, i} \widehat{G}_{i, i+1}-\widehat{G}_{k, i+1}, & k \neq i, i+1, \\ \widehat{\widehat{G}}_{i, i+1}=\widehat{G}_{i, i+1}, & \\ \widetilde{\widehat{G}}_{i+1, i+1}=\widehat{G}_{i, i}, & \\ \widetilde{\widehat{G}}_{i, i}=\widehat{G}_{i, i} \widehat{G}_{i, i+1}-\widehat{G}_{i+1, i+1}, & \\ \widetilde{\widehat{G}}_{i+1, i}=\widehat{G}_{i+1, i}+\widehat{G}_{i, i+1} \widehat{G}_{i, i}^{2}-2 \widehat{G}_{i, i} \widehat{G}_{i+1, i+1}\end{cases}
$$

для $1 \leqslant i \leqslant n-1$ и

$$
\beta_{n, 1} \widehat{G}_{k, l}=\widetilde{\widehat{G}}_{k, l}: \begin{cases}\widetilde{\widehat{G}}_{1, k}=\widehat{G}_{n, k}, & k \neq n, 1, \\ \widehat{\widehat{G}}_{n, k}=\widehat{G}_{n, k} \widehat{G}_{n, 1}-\widehat{G}_{1, k}, & k \neq n, 1, \\ \widetilde{\widehat{G}}_{k, 1}=\widehat{G}_{k, n}, & k \neq n, 1, \\ \widetilde{\widehat{G}}_{k, n}=\widehat{G}_{k, n} \widehat{G}_{n, 1}-\widehat{G}_{k, 1} & \\ \widetilde{\widehat{G}}_{n, 1}=\widehat{G}_{n, 1}, & \\ \widetilde{\widehat{G}}_{1,1}=\widehat{G}_{n, n}, & \\ \widetilde{\widehat{G}}_{n, n}=\widehat{G}_{n, n} \widehat{G}_{n, 1}-\widehat{G}_{1,1}, & \\ \widetilde{\widehat{G}}_{1, n}=\widehat{G}_{1, n}+\widehat{G}_{n, 1} \widehat{G}_{n, n}^{2}-2 \widehat{G}_{n, n} \widehat{G}_{1,1} .\end{cases}
$$

ЛЕмма 5.1. При любом $n \geqslant 2$ имеет место соотношение группы кос для преобразований (24), (25):

$$
\beta_{i-1, i} \beta_{i, i+1} \beta_{i-1, i}=\beta_{i, i+1} \beta_{i-1, i} \beta_{i, i+1}, \quad i=1, \ldots, n(\bmod n),
$$

где при $i=n$ преобразование $\beta_{n, n+1}$ есть $\beta_{n, 1}$.

Заметим, что дополнительное соотношение группы кос (22) не выполняется в случае алгебр $D_{n}$. Это связано с топологическими ограничениями, накладываемыми введением новой дырки.

Найти матричное (ковариантное) представление для полного действия группы кос в виде (21) оказалось нетривиальной задачей. Важным предварительным результатом оказывается тот факт, что специальные комбинации элементов $\widehat{G}_{i, j}$ преобразуются одинаково относительно действия подгруппы $A_{n}$, порождаемой элементами $\left\langle\beta_{1,2}, \ldots, \beta_{n-1, n}\right\rangle$ полной группы кос. 
Лемма 5.2 [12]. Рассмотрим антисимметричную $(n \times n)$-матрицу $\widehat{\mathscr{R}} c$ элементами

$$
(\widehat{\mathscr{R}})_{i, j}:= \begin{cases}-\widehat{G}_{j, i}-\widehat{G}_{i, j}+\widehat{G}_{i, i} \widehat{G}_{j, j}, & j<i, \\ \widehat{G}_{j, i}+\widehat{G}_{i, j}-\widehat{G}_{i, i} \widehat{G}_{j, j}, & j>i, \\ 0, & j=i,\end{cases}
$$

симметричную матрицу $\widehat{\mathscr{S}}$ с элементами

$$
(\widehat{\mathscr{S}})_{i, j}:=\widehat{G}_{i, i} \widehat{G}_{j, j}, \quad 1 \leqslant i, j \leqslant n,
$$

и верхнетреугольную матрицу $\widehat{\mathscr{A}}$ с элементами

$$
\widehat{\mathscr{A}}_{i, j}= \begin{cases}\widehat{G}_{i, j}, & i<j, \\ 0, & i>j, \\ 1, & i=j .\end{cases}
$$

Тогда произвольная линейная комбинация $w_{1} \widehat{\mathscr{A}}+w_{2} \widehat{\mathscr{A}}^{T}+\rho \widehat{\mathscr{R}}+\sigma \widehat{\mathscr{S}}$ скомплексными $w_{1}, w_{2}, \rho$ и $\sigma$ преобразуется в соответствии с формулой (21) относительно подгруппь $A_{n}$, порождаемой генераторами $\left\langle\beta_{1,2}, \ldots, \beta_{n-1, n}\right\rangle$ (24) полной группы кос.

В разделе 8 мы вернемся к задаче построения матричного представления для полной группы кос в случае алгебр $D_{n}$.

\section{6. Монодромии фуксовых систем}

Обратимся к интерпретации матриц $\gamma_{1}, \ldots, \gamma_{n}$ как матриц монодромий фуксовой системы с $(2 \times 2)$-матрицами вычетов $A_{j}$, не зависящими от $\lambda$ :

$$
\frac{\mathrm{d}}{\mathrm{d} \lambda} \Phi=\left(\sum_{k=1}^{n} \frac{A_{k}}{\lambda-u_{k}}\right) \Phi
$$

где $\mathbf{u}=\left(u_{1}, \ldots, u_{n}\right)$ - попарно различные точечные вершины ленточного графа, а матрицы $A_{j}$ удовлетворяют условиям

$$
\text { собственные значения }\left(A_{j}\right)= \pm \frac{1}{4} \quad \text { и } \quad-\sum_{k=1}^{n} A_{k}=A_{\infty} .
$$

ТеОрема 6.1 [28]. Пусть даны $n$ произвольных $(2 \times 2)$-матрии, $M_{1}, \ldots, M_{n}$ и произвольное число $\mu$ такое, что

$$
M_{\infty}:=\left(M_{1} M_{2} \cdots M_{n-1} M_{n}\right)^{-1}
$$

имеет вид $\left(\begin{array}{cc}e^{2 i \pi \mu} & 0 \\ 0 & e^{-2 i \pi \mu}\end{array}\right)$ рри $\mu \notin \mathbb{Z} / 2,\left(\begin{array}{cc}1 & 1 \\ 0 & 1\end{array}\right)$ при $\mu \in \mathbb{Z} u\left(\begin{array}{cc}-1 & 1 \\ 0 & -1\end{array}\right) n p u$ $\mu \in 1 / 2+\mathbb{Z}$, и дана точка $\mathbf{u}^{0}=\left(u_{1}^{0}, \ldots, u_{n}^{0}\right) \in X_{n}, X_{n}:=\mathbb{C}^{n} \backslash\{$ diagonals $\}$. Tогда в прочзвольной окрестности $U \subset X_{n}$ точки $\mathbf{u}^{0}$ существует точка $\mathbf{u} \in U$ и фуксова система

$$
\frac{\mathrm{d}}{\mathrm{d} \lambda} \Phi=\left(\sum_{k=1}^{n} \frac{A_{k}}{\lambda-u_{k}}\right) \Phi
$$


матрицы монодромии $M_{1}, \ldots, M_{n}$ которой имеют заданный вид, а монодромия на бесконечности $A_{\infty}$ выражается следующим образом:

$$
\begin{array}{ll}
A_{\infty}:=\left(\begin{array}{cc}
\mu & 0 \\
0 & -\mu
\end{array}\right), & \text { если } \mu \neq 0, \\
A_{\infty}:=\left(\begin{array}{ll}
0 & 1 \\
0 & 0
\end{array}\right), \quad \text { если } \mu=0 .
\end{array}
$$

Существует целое семейство фуксовых систем с совпадающими матрицами монодромий, они задаются решениями уравнений Шлезингера [29], [30]. Так как в этой работе нас интересуют алгебры монодромий, мы не рассматриваем изомонодромные деформации; по поводу уравнений Шлезингера см. [9].

6.1. Действие группы кос. Процедура аналитического продолжения для решений уравнений Шлезингера в терминах действия группы кос $\mathscr{B}_{n}=$ $\left\langle\beta_{1}, \ldots, \beta_{n-1}\right\rangle$ на матрицах монодромий $M_{1}, \ldots, M_{n}$ была построена в работе [31]. Это действие имеет вид

$$
\begin{aligned}
& \beta_{i, i+1}\left(M_{j}\right)=M_{j}, \quad j=1, \ldots, i-1, i+2, \ldots, n, \\
& \beta_{i, i+1}\left(M_{i}\right)=M_{i} M_{i+1} M_{i}^{-1}, \quad \beta_{i, i+1}\left(M_{i+1}\right)=M_{i} .
\end{aligned}
$$

С помощью распутывающих соотношений можно непосредственно показать, что на элементах $G_{i, j}:=-\operatorname{Tr}\left(M_{i} M_{j}\right)$ действие группы кос совпадает с действием (19), так что действие группы классов отображений на пространстве Тейхмюллера диска Пуанкаре с $n \mathbb{Z}_{2}$-орбифолдными точками отвечает процедуре аналитического продолжения для соответствующего решения уравнений Шлезингера.

6.2. Скобка Короткина-Самтлебена. Стандартная скобка ПуассонаЛи на $\mathfrak{g}^{*}$ представима в $r$-матричном виде:

$$
\left\{A\left(\lambda_{1}\right) \otimes, A\left(\lambda_{2}\right)\right\}=\left[\stackrel{1}{A}\left(\lambda_{1}\right)+\stackrel{2}{A}\left(\lambda_{2}\right), r\left(\lambda_{1}-\lambda_{2}\right)\right],
$$

где $r(z)=\Omega / \lambda-$ классическая $r$-матрища, т. е. решение классического уравнения Янга-Бакстера. В случае $\mathfrak{g}:=\bigoplus_{n} \mathfrak{s l}(m)$ матрица $\Omega$ задается матрицей перестановок $\Omega=\sum_{i, j} \stackrel{1}{E}_{i, j} \otimes \stackrel{2}{E}_{j, i}$, если отождествить алгебру $\mathfrak{s l}(m)$ с дуальной алгеброй с помощью формы Киллинга: $(A, B)=\operatorname{Tr} A B, A, B \in \mathfrak{s l}(m)$.

Стандартная скобка Пуассона-Ли на $\mathfrak{s l}(m, \mathbb{C})$ с помощью соответствия Римана-Гильберта отображается в скобку Короткина-Самтлебена:

$$
\begin{aligned}
& \left\{M_{i} \otimes M_{i}\right\}=\frac{1}{2}\left(\stackrel{2}{M_{i}} \stackrel{1}{M_{i}}-\stackrel{1}{M_{i}} \Omega \stackrel{2}{M_{i}}\right), \\
& \left\{M_{i} \otimes, M_{j}\right\}=\frac{\varepsilon(i-j)}{2}\left(\stackrel{1}{M_{i}} \Omega \stackrel{2}{M_{j}}+\stackrel{2}{M_{j}} \Omega \stackrel{1}{M_{i}}-\stackrel{1}{M_{i}} \stackrel{2}{M_{j}}-\stackrel{2}{M_{j}} \stackrel{1}{M_{i} \Omega}\right), \quad i \neq j
\end{aligned}
$$

$(\varepsilon(\cdot)$ - знаковая функция \pm 1$)$. Хотя сама по себе эта скобка не удовлетворяет тождеству Якоби, она становится скобкой Пуассона при ограничении на величины, инвариантные относительно сопряжений. 
Лемма 6.2 [9]. Пуассонова алгебра $A_{n}(16)$ представляет собой скобку Короткина-Самтлебена, суженную на элементы, инвариантные относительно сопря⿻ений. ${ }^{5}$

$$
G_{i, j}:=-\operatorname{Tr}\left(\gamma_{i} \gamma_{j}\right)=-\operatorname{Tr}\left(M_{i} M_{j}\right)
$$

ДокАзАТЕЛЬСтво. Покажем, как доказывается соотношение

$$
\left\{G_{i, k}, G_{j, l}\right\}=2\left(G_{i, j} G_{k, l}-G_{i, l} G_{k, j}\right), \quad i<j<k<l .
$$

По определению величин $G_{i, j}$ имеем:

$$
\begin{aligned}
& \left\{G_{i, k}, G_{j, l}\right\}=\left\{\operatorname{Tr}\left(M_{i} M_{k}\right), \operatorname{Tr}\left(M_{j} M_{l}\right)\right\} \\
& =\stackrel{12}{\operatorname{Tr}}\left(\left\{\stackrel{1}{M_{i}} \otimes \stackrel{2}{M_{j}}\right\} \stackrel{1}{M_{k}} \stackrel{2}{M_{l}}+\stackrel{2}{M_{j}}\left\{\stackrel{1}{M_{i}} \stackrel{\stackrel{2}{M_{l}}}{{ }}\right\} \stackrel{1}{M_{k}}\right. \\
& \left.+\stackrel{1}{M}\left\{\stackrel{1}{M_{k}} \otimes \stackrel{2}{M_{j}}\right\} \stackrel{2}{M_{l}}+\stackrel{1}{M_{i}} \stackrel{2}{M_{j}}\left\{\stackrel{1}{M_{k}} \otimes \stackrel{2}{M_{l}}\right\}\right) \\
& =\frac{1}{2}(\varepsilon(i-j)-\varepsilon(i-l)-\varepsilon(k-j)+\varepsilon(k-l)) \operatorname{Tr}\left(\left[M_{k}, M_{i}\right]\left[M_{j}, M_{l}\right]\right) .
\end{aligned}
$$

В случае матриц размера $2 \times 2$, учитывая то, что $M_{a}^{2}=-\mathbb{E}$ для любого $a$ и, соответственно, $\left(M_{a} M_{b}\right)^{-1}=M_{b} M_{a}$, имеем, например,

$$
\begin{aligned}
\operatorname{Tr}\left(\left[M_{k}, M_{i}\right] M_{j} M_{l}\right) & =\operatorname{Tr}\left(M_{k} M_{i} M_{j} M_{l}-M_{i} M_{k} M_{j} M_{l}\right) \\
& =\operatorname{Tr}\left(M_{i} M_{j}\right) \operatorname{Tr}\left(M_{l} M_{k}\right)-\operatorname{Tr}\left(M_{k} M_{j} M_{i} M_{l}+M_{i} M_{k} M_{j} M_{l}\right) \\
& =\operatorname{Tr}\left(M_{i} M_{j}\right) \operatorname{Tr}\left(M_{l} M_{k}\right)-\operatorname{Tr}\left(M_{k} M_{j}\left(M_{i} M_{l}+M_{l} M_{i}\right)\right) \\
& =\operatorname{Tr}\left(M_{i} M_{j}\right) \operatorname{Tr}\left(M_{l} M_{k}\right)-\operatorname{Tr}\left(M_{k} M_{j}\right) \operatorname{Tr}\left(M_{i} M_{l}\right),
\end{aligned}
$$

так что

$$
\operatorname{Tr}\left(\left[M_{k}, M_{i}\right]\left[M_{j}, M_{l}\right]\right)=2 \operatorname{Tr}\left(M_{i} M_{j}\right) \operatorname{Tr}\left(M_{l} M_{k}\right)-2 \operatorname{Tr}\left(M_{k} M_{j}\right) \operatorname{Tr}\left(M_{i} M_{l}\right),
$$

и в итоге получим скобку (34). Лемма 6.2 доказана.

6.3. Процедура слияния полюсов. Рассмотрим теперь фуксову систему вида (30) с матрицами монодромий $M_{1}, \ldots, M_{n}$ размера $2 \times 2$ и исследуем ее связь с новой фуксовой системой

$$
\frac{\mathrm{d}}{\mathrm{d} \lambda} \widetilde{\Phi}=\left(\sum_{k=1}^{\tilde{n}} \frac{\widetilde{A}_{k}}{\lambda-u_{k}}\right) \widetilde{\Phi},
$$

в которой $\tilde{n}=n-m+1$ при некотором положительном целом числе $m<n$, и с новыми матрицами монодромий $\widetilde{M}_{1}, \ldots, \widetilde{M}_{\tilde{n}}$, где

$$
\tilde{M}_{i}=M_{i}, \quad i=1, \ldots, \tilde{n}-1, \quad \text { и } \quad \tilde{M}_{\tilde{n}}=M_{n-m+1} \cdots M_{n-1} M_{n} .
$$

\footnotetext{
${ }^{5}$ Пуассонова алгебра (16) была получена в работе [7] как сужение скобки КороткинаСамтлебена на следы произведений матриц монодромий размерности $n \times n$.
} 
Основная идея состоит в том, что система (36) может быть получена из системы (30) с помощью слияния $m$ полюсов [32]. Это достигается, если положить

$$
\begin{aligned}
\widetilde{\mathbf{u}} & :=\left(u_{1}, \ldots, u_{\tilde{n}-1}\right), \\
u_{j} & :=t v_{j}, \quad j=\tilde{n}, \ldots, \tilde{n}+m-1=n,
\end{aligned}
$$

и

$$
\begin{array}{rlrl}
A_{i}(\widetilde{\mathbf{u}}, t) & :=A_{i}\left(\widetilde{\mathbf{u}}, t v_{\tilde{n}}, \ldots, t v_{n}\right), & & i=1, \ldots, \tilde{n}-1, \\
B_{j}(\widetilde{\mathbf{u}}, t):=A_{\tilde{n}-1+j}\left(\widetilde{\mathbf{u}}, t v_{\tilde{n}}, \ldots, t v_{n}\right), & j=1, \ldots, m .
\end{array}
$$

Следующая теорема устанавливает условия, при которых система (36) может быть получена в пределе $t \rightarrow 0$ из системы (30). Сформулируем эти условия в полной общности для произвольной размерности $m$ исследуемой фуксовой системы.

Теорема 6.3 [32]. Пусть $A_{1}^{0}, \ldots, A_{\tilde{n}-1}^{0}, B_{1}^{0}, \ldots, B_{m}^{0}$ - некоторые не зависящие от переменной $t$ матрицы такие, что

$$
\Lambda:=\sum_{j=1}^{m} B_{j}^{0}
$$

имеет собственные значения $\lambda_{1}, \ldots, \lambda_{n}$ такие, что удовлетворяются следующие технические предположения:

$$
\vartheta:=\max _{i, j=1, \ldots, m}\left\{\left|\operatorname{Re} \lambda_{i}-\operatorname{Re} \lambda_{j}\right|\right\} \in[0,1[,
$$

и пусть $K$ - константа такая, что

$$
\left|A_{i}^{0}\right|<K, \quad i=1, \ldots, \tilde{n}-1, \quad\left|B_{j}^{0}\right|<K, \quad j=1, \ldots, m .
$$

Тогда верны следующие утверждения.

(i) Пусть $\Phi(\lambda, t)$ - решение системь (30), нормированное на бесконечности. Тогда предел $\widetilde{\Phi}(\lambda):=\lim _{t \rightarrow 0} \Phi(\lambda, t)$ существует и удовлетворяет системе $(36) c$

$$
u_{\tilde{n}}=0, \quad \widetilde{A}_{i}=A_{i}^{0}, \quad i=1, \ldots, \tilde{n}-1, \quad \widetilde{A}_{\tilde{n}}=\Lambda .
$$

(ii) Соответствующие матрицы монодромий системъ (36) в предположении выполнения условий (39) имеют вид $\widetilde{M}_{1}, \ldots, \widetilde{M}_{\tilde{n}}$, где

$$
\tilde{M}_{i}=M_{i}, \quad i=1, \ldots, \tilde{n}-1, \quad u \quad \tilde{M}_{\tilde{n}}=M_{n-m+1} \cdots M_{n-1} M_{n} .
$$

В нашем случае, т. е. когда система (30) получается из алгебры $A_{n}$, всегда можно объединить нечетное число полюсов. В действительности для нечетного числа $m=n-\tilde{n}+1$

$$
\operatorname{Tr}\left(M_{n-m+1} \cdots M_{n-1} M_{n}\right)=\operatorname{Tr}\left(\gamma_{n-m+1} \cdots \gamma_{n-1} \cdots \gamma_{n}\right)=2 \cos (\pi \vartheta)
$$

всегда представляет собой целое число, бо́льшее 2. Следовательно, величина $\vartheta$ чисто мнимая $(\operatorname{Re} \vartheta=0)$, и условия теоремы 6.3 выполнены. 


\section{7. Алгебра $\mathfrak{D}_{n}$}

В этом разделе система (36) интерпретируется как система на кольце с $n$ $\mathbb{Z}_{2}$-орбифолдными точками, в то время как дырка в центре возникает в результате слияния $m$ полюсов. Для удобства изменим обозначения: начнем с системы с $n+m$ полюсами и объединим $m$ из этих полюсов, сохранив оставшиеся $n$ орбифолдных точек. Матрица монодромии $M_{h}$ обхода вокруг дырки примет вид

$$
M_{h}=M_{n+1} \cdots M_{n+m-1} M_{n+m} .
$$

Теперь в алгебру Пуассона будут давать вклады пути, соединяющие точки $u_{i}$ и $u_{j}$ и обкручивающиеся $k$ раз (возможно, с самопересечениями) вокруг периметра новой дырки. Следы соответствующих элементов в фуксовой группе имеют вид

$$
G_{i, j}^{(k)}:=-\operatorname{Tr}\left(M_{i} M_{h}^{k} M_{j} M_{h}^{-k}\right) .
$$

Заметим, что

$$
G_{i, j}^{(k)}=G_{j, i}^{(-k)}
$$

так что, в частности, элементы нулевого уровня $G_{i, j}^{(0)}$ симметричны и

$$
G_{i, i}^{(0)}=2 .
$$

Пуассонова алгебра элементов $G_{i, j}^{(k)}$ описывается следующей теоремой.

ТеОрема 7.1 [9]. Геодезические функиии $G_{i, j}^{(k)}$ удовлетворяют следующим пуассоновым соотношениям: при $0 \leqslant k$

$$
\begin{aligned}
\left\{G_{j, i}^{(0)}, G_{p, l}^{(k)}\right\}=( & (j-l)-\varepsilon(i-l))\left(G_{l, i}^{(0)} G_{p, j}^{(k)}-G_{l, j}^{(0)} G_{p, i}^{(k)}\right) \\
& +(\varepsilon(j-p)-\varepsilon(i-p))\left(G_{p, i}^{(0)} G_{j, l}^{(k)}-G_{p, j}^{(0)} G_{i, l}^{(k)}\right),
\end{aligned}
$$

a при $0<m \leqslant k$

$$
\begin{aligned}
& \left\{G_{j, i}^{(m)}, G_{p, l}^{(k)}\right\} \\
& =\varepsilon(i-l)\left(G_{p, i}^{(k)} G_{j, l}^{(m)}-G_{i, l}^{(0)} G_{p, j}^{(k-m)}\right)+\varepsilon(i-p)\left(G_{j, p}^{(m)} G_{i, l}^{(k)}-G_{i, p}^{(0)} G_{j, l}^{(k+m)}\right) \\
& \quad+\varepsilon(j-l)\left(G_{p, j}^{(k)} G_{l, i}^{(m)}-G_{j, l}^{(0)} G_{p, i}^{(k+m)}\right)+\varepsilon(j-p)\left(G_{p, i}^{(m)} G_{j, l}^{(k)}-G_{j, p}^{(0)} G_{i, l}^{(k-m)}\right) \\
& \quad+\left[G_{p, i}^{(k+m)} G_{j, l}^{(0)}+2 G_{p, i}^{(k+m-1)} G_{j, l}^{(1)}+\cdots+2 G_{p, i}^{(k+1)} G_{j, l}^{(m-1)}+G_{p, i}^{(k)} G_{j, l}^{(m)}\right. \\
& \quad-G_{p, i}^{(m)} G_{j, l}^{(k)}-2 G_{p, i}^{(m-1)} G_{j, l}^{(k+1)}-\cdots-2 G_{p, i}^{(1)} G_{j, l}^{(k+m-1)}-G_{p, i}^{(0)} G_{j, l}^{(k+m)} \\
& \quad+G_{i, l}^{(k-m)} G_{j, p}^{(0)}+2 G_{i, l}^{(k-m+1)} G_{j, p}^{(1)}+\cdots+2 G_{i, l}^{(k-1)} G_{j, p}^{(m-1)}+G_{i, l}^{(k)} G_{j, p}^{(m)} \\
& \\
& \left.\quad-G_{l, i}^{(0)} G_{p, j}^{(k-m)}-2 G_{l, i}^{(1)} G_{p, j}^{(k-m+1)}-\cdots-2 G_{l, i}^{(m-1)} G_{p, j}^{(k-1)}-G_{l, i}^{(m)} G_{p, j}^{(k)}\right],
\end{aligned}
$$

¿əe

$$
\varepsilon(x)= \begin{cases}-1, & x<0 \\ 0, & x=0 \\ 1, & x>0\end{cases}
$$


Введем производящую функиию

$$
\mathscr{G}_{i, j}(\lambda):=\mathscr{A}_{i, j}^{(0)}+\sum_{k=1}^{\infty} G_{i, j}^{(k)} \lambda^{-k},
$$

где $\mathscr{A}^{(0)}$ - верхнетреугольная матрица с элементами

$$
\mathscr{A}_{i, j}^{(0)}= \begin{cases}G_{i, j}^{(0)}, & i<j, \\ 1, & i=j, \\ 0, & i>j .\end{cases}
$$

Скобки Пуассона при этом принимают вид

$$
\begin{aligned}
\left\{\mathscr{G}_{j, i}(\lambda), \mathscr{G}_{p, l}(\mu)\right\}=( & \left.\varepsilon(j-p)-\frac{\lambda+\mu}{\lambda-\mu}\right) \mathscr{G}_{p, i}(\lambda) \mathscr{G}_{j, l}(\mu) \\
& +\left(\varepsilon(i-l)+\frac{\lambda+\mu}{\lambda-\mu}\right) \mathscr{G}_{p, i}(\mu) \mathscr{G}_{j, l}(\lambda) \\
& +\left(\varepsilon(i-p)-\frac{1+\lambda \mu}{1-\lambda \mu}\right) \mathscr{G}_{j, p}(\lambda) \mathscr{G}_{i, l}(\mu) \\
& +\left(\varepsilon(j-l)+\frac{1+\lambda \mu}{1-\lambda \mu}\right) \mathscr{G}_{l, i}(\lambda) \mathscr{G}_{p, j}(\mu) .
\end{aligned}
$$

Эти скобки задают абстрактную бесконечномерную пуассонову алгебру.

Заметим, что алгебра Нельсон-Редже (16) представляет собой подалгебру алгебры (47), образованную элементами нулевого уровня $G_{i, j}^{(0)}$.

Подчеркнем, что число $m$ объединяемых полюсов не появляется нигде в вышеприведенных формулах. В самом деле, если интерпретировать элементы $G_{i, j}^{(k)}$ как бесконечное число независимых элементов, то скобки (43) и (44) задают пуассонову алгебру, удовлетворяющую тождествам Якоби.

ОПРЕДЕЛЕНИЕ 7.2. Будем называть пуассоновы соотношения (43) и (44) aлгеброй $\mathfrak{D}_{n}$ элементов $G_{i, j}^{(k)}$.

Подчеркнем основные моменты доказательства теоремы 7.1. Сначала строится пуассонова алгебра элементов $G_{i, j}^{(k)}$, рассматриваемых как элементы подгруппы $\Delta_{0,1, n+m, 0}-$ фуксовой группы в случае алгебры $A_{n+m}$. Выполнение тождеств Якоби проверяется при этом прямым вычислением, выполненным в работе [9].

Доказательство также основывается на примечательном наблюдении, что скобку Короткина-Самтлебена можно расширить на матрицы монодромий системы (36):

ПРЕДЛОЖЕНИЕ 7.3. Если $M_{i}, i=1, \ldots, n+m$, удовлетворяют соотношениям Пуассона (33), то набор новых матрии, $\tilde{M}_{i}, i=1, \ldots, n+1$, такой, что $\widetilde{M}_{i}=M_{i}, i=1, \ldots, n, u \widetilde{M}_{n+1}=M_{h}$, удовлетворяет тем же самым 
соотношениям (33). Кроме того, имеют место следующие соотношения для степеней матрицы $M_{h}:$ для любого $k \in \mathbb{Z}$

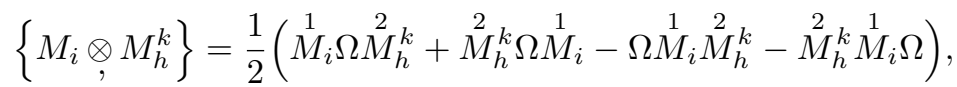

$$
\begin{aligned}
& \left\{M_{h} \otimes, M_{h}^{k}\right\}=\frac{1}{2}\left(\stackrel{2}{M_{h}^{k}} \stackrel{\stackrel{1}{M}}{M_{h}}+\stackrel{1}{M_{h}} \stackrel{2}{M_{h}^{k}} \Omega-\stackrel{1}{M_{h}} \stackrel{2}{M_{h}^{k}}-\stackrel{1}{M_{h}} \Omega \stackrel{2}{M_{h}^{k}}\right) .
\end{aligned}
$$

В п. 7.2 будет показано, что алгебра $\mathfrak{D}_{n}$ представляет собой квазиклассический предел твистованного $q$-янгиана $Y_{q}^{\prime}\left(\mathfrak{o}_{n}\right)$ для ортогональной алгебры Ли $\mathfrak{o}_{n}$, введенного в работе [10]. Это в свою очередь предполагает выполнение тождеств Якоби.

Рассмотрим пример дырки, образующейся при слиянии двух полюсов ${ }^{6}$.

7.1. Пример: слияние двух полюсов. Начнем с алгебры $A_{n+2}$ и будем интерпретировать элемент $M_{h}=M_{n+1} M_{n+2}$ как элемент, отвечающий обходу вокруг новой дырки. Рассмотрим фуксову группу $\Delta_{0,2, n}$, порождаемую $M_{h}$ и $M_{i}, i=1, \ldots, n$. Построенная таким образом группа, очевидно, будет подгруппой группы $\Delta_{0,1, n+2}$, а именно фуксовой группы в случае алгебры $A_{n+2}$. Периметр новой дырки образован петлей, охватывающей два объединяемых полюса $u_{n+1}$ и $u_{n+2}$. Это означает, что следует заменить древовидный подграф в левой части рис. 14 на петлеобразный подграф в правой части того же рисунка. Произведение матриц, отвечающих путям, обходящим вокруг точек $u_{n+1}$

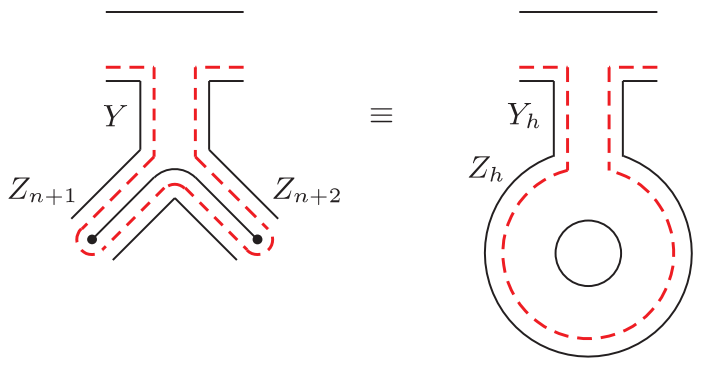

Рис. 14

и $u_{n+2}$ в левой части рисунка, при этом сохраняется: имеет место матричное равенство

$$
X_{Y} R X_{Z_{n+2}} F X_{Z_{n+2}} R X_{Z_{n+1}} F X_{Z_{n+1}} R X_{Y}=X_{Y_{h}} R X_{Z_{h}} R X_{Y_{h}},
$$

которое выполняется, если новые координаты $Z_{h}$ и $Y_{h}$ удовлетворяют условиям

$$
\begin{gathered}
Y+Z_{n+1}+Z_{n+2}=Y_{h}+\frac{Z_{h}}{2}, \\
G_{n+1, n+2}=e^{Z_{n+1}+Z_{n+2}}+e^{-Z_{n+1}-Z_{n+2}}+e^{Z_{n+1}-Z_{n+2}}=e^{Z_{h} / 2}+e^{-Z_{h} / 2} .
\end{gathered}
$$

Элемент $G_{n+1, n+2}$, очевидно, коммутирует со всеми элементами из (под)группы $\Delta_{0,2, n}$.

\footnotetext{
${ }^{6} \mathrm{~B}$ геометрическом подходе нет ограничения на число объединяемых полюсов; условие нечетности $m$ возникает только в случае, когда требуется проследить слияние полюсов в аналитическом подходе.
} 
То, что фуксову группу $\Delta_{0,2, n, 0}$, порождаемую $M_{h}$ и $M_{i}, i=1, \ldots, n$, можно рассматривать как подгруппу $\Delta_{0,1, n+2,0}$ - фуксовой группы в случае алгебры $A_{n+2}$, можно показать на примере элементов $G_{i, j}^{(k)}$, которые могут быть выражены в терминах элементов из группы $\Delta_{0,1, n+2,0}$. Например, соотношение

$$
G_{i, j}^{(1)}=G_{i, n+2} G_{n+1, n+2} G_{j, n+1}-G_{i, n+1} G_{j, n+1}-G_{i, n+2} G_{j, n+2}+G_{i, j}
$$

следует из соотношений распутывания (12), см. рис. 15.
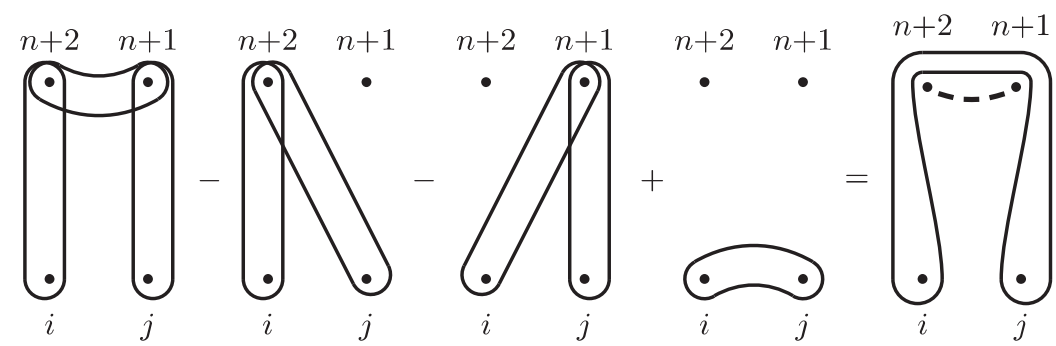

Рис. 15

7.2. Алгебра $\mathfrak{D}_{n}$ как квазиклассический предел $Y_{q}^{\prime}\left(\mathfrak{o}_{n}\right)$ [9]. Покажем теперь, что алгебра $\mathfrak{D}_{n}$ представляет собой квазиклассический предел твистованной $q$-янгианной алгебры $Y_{q}^{\prime}\left(\mathfrak{o}_{n}\right)$ для алгебры Ли ортогональных матриц $\mathfrak{o}_{n}$, введенной в работе [10]. Янгианная алгебра задается матричнозначными элементами $G_{i, j}^{(k)}, i, j=1, \ldots, n, k \in \mathbb{Z}_{\geqslant 0}$, подчиняющимися определяющим соотношениям:

$$
R(\lambda, \mu) \stackrel{1}{\mathscr{G}}(\lambda) R\left(\lambda^{-1}, \mu\right)^{T_{1}} \stackrel{2}{\mathscr{G}}(\mu)=\stackrel{2}{\mathscr{G}}(\mu) R\left(\lambda^{-1}, \mu\right)^{T_{1}} \stackrel{1}{\mathscr{G}}(\lambda) R(\lambda, \mu),
$$

в которых $R$-матрица имеет вид

$$
\begin{aligned}
R(\lambda, \mu)=(\lambda & -\mu) \sum_{i \neq j} E_{i, i} \otimes E_{j, j}+\left(q^{-1} \lambda-q \mu\right) \sum_{i} E_{i, i} \otimes E_{i, i} \\
& +\left(q^{-1}-q\right) \lambda \sum_{i<j} E_{i, j} \otimes E_{j, i}+\left(q^{-1}-q\right) \mu \sum_{i>j} E_{i, j} \otimes E_{j, i}
\end{aligned}
$$

индекс $T_{1}$ обозначает транспонирование в пространстве с номером один. Эта $R$-матрица удовлетворяет уравнению Янга-Бакстера.

Квазиклассический предел получится, если положить $q=-\exp (i \pi \hbar)$ и собрать члены первого порядка по $\hbar$ при $\hbar \rightarrow 0$. Разложение для $R$-матрицы имеет вид

$$
R(\lambda, \mu)=(\lambda-\mu) \mathbb{E} \otimes \mathbb{E}+i \pi \hbar r(\lambda, \mu),
$$


где $r$ - классическая $r$-матрица:

$$
r(\lambda, \mu)=(\lambda+\mu) \sum E_{i, i} \otimes E_{i, i}+2 \lambda \sum_{i<j} E_{i, j} \otimes E_{j, i}+2 \mu \sum_{i>j} E_{i, j} \otimes E_{j, i}
$$

в то время как матрица $\mathscr{G}(\lambda)$ не меняется. Уравнение отражения (53) в квазиклассическом пределе принимает вид

$$
\begin{aligned}
& \{\stackrel{1}{\mathscr{G}}(\lambda) \stackrel{2}{\stackrel{2}{\mathscr{G}}(\mu)}\}=\left[\frac{r(\lambda, \mu)}{\lambda-\mu}, \stackrel{1}{\mathscr{G}}(\lambda) \stackrel{2}{\mathscr{G}}(\mu)\right]+\stackrel{1}{\mathscr{G}}(\lambda) \frac{r\left(\lambda^{-1}, \mu\right)^{T_{1}}}{\lambda^{-1}-\mu} \stackrel{2}{\mathscr{G}}(\mu) \\
& -\stackrel{2}{\mathscr{G}}(\mu) \frac{r\left(\lambda^{-1}, \mu\right)^{T_{1}}}{\lambda^{-1}-\mu} \stackrel{1}{\mathscr{G}}(\lambda) \text {. }
\end{aligned}
$$

С помощью прямого вычисления можно показать, что формула (56) совпадает c $(47)^{7}$.

7.3. Соотношения группы кос для $\mathfrak{D}_{n}$. Группа кос (равно как и группа классов отображений) в случае алгебры $\mathfrak{D}_{n}$ порождается $n$ генераторами. Помимо стандартных генераторов $\beta_{i, i+1}, i=1, \ldots, n-1$, каждый из которых переставляет местами орбифолдные точки с номерами $i$ и $i+1$, возникает еще один генератор $\beta_{1, n}$, переставляющий первую и последнюю (номер $n$ ) орбифолдные точки. Действие этого элемента не представимо через предыдущие ввиду наличия новой дырки:

Теорема 7.4. Обозначим через $\mathscr{G}^{(k)}$ матрицу с элементами $G_{i, j}^{(k)}$. Действие генераторов группы кос $\beta_{i, i+1}$ при $i=1, \ldots, n-1$ задается формулой

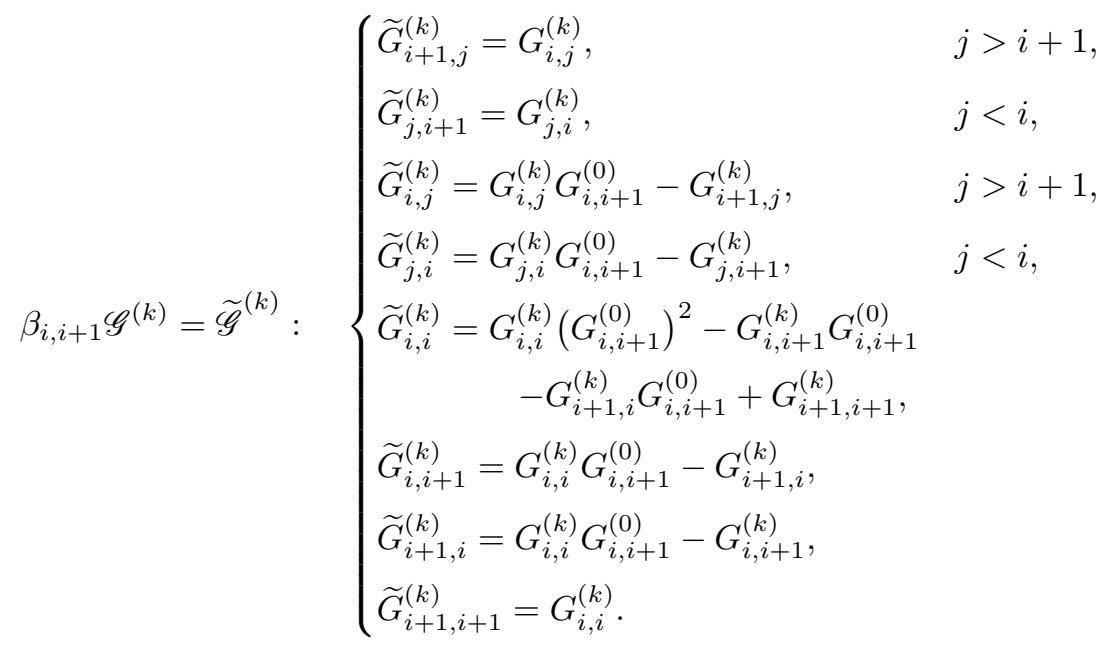

\footnotetext{
${ }^{7} \mathrm{~B}$ работе [10] использовалась другая нормировка для $\mathscr{G}(\lambda)$ : нулевой уровень выбирался нижнетреугольным, вследствие чего $R$-матрица была глобально транспонирована.
} 
Действие последнего генератора $\beta_{n, 1}$ имеет вид

$$
\beta_{n, 1} \mathscr{G}^{(k)}=\widetilde{\mathscr{G}}^{(k)}: \begin{cases}\widetilde{G}_{1, j}^{(k)}=G_{n, j}^{(k+1)}, & 1<j<n, \\ \widetilde{G}_{j, 1}^{(k)}=G_{j, n}^{(k-1)}, & 1<j<n, \\ \widetilde{G}_{n, j}^{(k)}=G_{n, j}^{(k)} G_{n, 1}^{(1)}-G_{1, j}^{(k-1)}, & 1<j<n, \\ \widetilde{G}_{j, n}^{(k)}=G_{j, n}^{(k)} G_{n, 1}^{(1)}-G_{j, 1}^{(k+1)}, & 1<j<n, \\ \widetilde{G}_{n, n}^{(k)}=G_{n, n}^{(k)}\left(G_{n, 1}^{(1)}\right)^{2}-G_{n, 1}^{(k+1)} G_{n, 1}^{(1)} & \\ -G_{1, n}^{(k-1)} G_{n, 1}^{(1)}+G_{1,1}^{(k)}, & \\ \widetilde{G}_{n, 1}^{(k)}=G_{n, n}^{(k-1)} G_{n, 1}^{(1)}-G_{1, n}^{(k-2)}, & \\ \widetilde{G}_{1, n}^{(k)}=G_{n, n}^{(k+1)} G_{n, 1}^{(1)}-G_{n, 1}^{(k+2)}, & \\ \widetilde{G}_{1,1}^{(k)}=G_{n, n}^{(k)}, & \end{cases}
$$

где предполагается выполнение соотношений (41) и (42), в частности, имеем:

$$
\widetilde{G}_{n, 1}^{(1)}=G_{n, n}^{(0)} G_{n, 1}^{(1)}-G_{1, n}^{(-1)}=G_{n, 1}^{(1)}
$$

Доказательство [9] основывается на рассмотрении действия группы кос (32) на матрицах монодромий $M_{1}, \ldots, M_{n}, M_{h}$.

ЗАмЕчАниЕ 7.5. Действие группы кос (57), (58) не зависит от числа $m$ объединяемых полюсов, а потому оно хорошо определено также и в случае абстрактной алгебры.

Заметим, что действие генераторов группы кос $\beta_{i, i+1}$ при $i=1, \ldots, n-1$ задается отдельно для каждой $(n \times n)$-матрицы $\mathscr{G}^{(k)}$ (т. е. все преобразования происходят внутри каждого из уровней $k$ ) и для каждой такой матрицы оно имеет один и тот же вид, в то время как действие последнего генератора $\beta_{n, 1}$ перемешивает различные уровни. Можно непосредственно доказать справедливость следующего предложения.

ПРЕДЛОЖЕНИЕ 7.6. Преобразования группъ кос для алгебры $\mathfrak{D}_{n}$ можно представить в матричном виде в терминах матрицы $\mathscr{G}(\lambda)(45)$ :

$$
\beta_{i, i+1} \mathscr{G}(\lambda)=B_{i, i+1} \mathscr{G}(\lambda) B_{i, i+1}^{T}, \quad i=1, \ldots, n-1,
$$

где матрицы $B_{i, i+1}$ зависят только от $G_{i, j}^{(0)}$ и имеют вид (20) (с элементом $G_{i, j}$, замененным на $\left.G_{i, j}^{(0)}\right)$. Действие генератора $\beta_{n, 1}$ записъвается в виде

$$
\beta_{n, 1} \mathscr{G}(\lambda)=B_{n, 1}(\lambda) \mathscr{G}(\lambda)\left(B_{n, 1}\left(\lambda^{-1}\right)\right)^{T}
$$


где

$$
B_{n, 1}(\lambda)=\left(\begin{array}{ccccc}
0 & 0 & \ldots & 0 & \lambda \\
0 & 1 & 0 & \ldots & 0 \\
\vdots & 0 & \ddots & \ddots & \vdots \\
0 & \vdots & \ddots & 1 & 0 \\
-\lambda^{-1} & 0 & \ldots & 0 & G_{n, 1}^{(1)}
\end{array}\right)
$$

\section{8. Центральные элементы алгебр $\mathfrak{D}_{n}$ и их пуассоновых редукций}

В силу предложения 7.6 полное действие группы кос представимо в матричном виде, а потому результат А. Молева и Э. Рагуци [13] о том, что центральные элементы алгебры $\mathfrak{D}_{n}$ задаются коэффициентами в разложении по $\lambda$ многочлена

$$
\operatorname{det} \mathscr{G}(\lambda)
$$

остается справедливым и в случае учета полного действия группы кос.

Мы будем активно использовать то обстоятельство, что общие центральные элементы алгебры Пуассона одновременно являются инвариантами группы кос (и инвариантами действия группы классов отображений в геометрическом подходе).

Рассмотрим теперь два типа конечномерных редукций: редукцию на уровне $p$ и редукиию к алгебре $D_{n}$ (см. п. 5.2), и построим действие группы кос и центральные элементы. Прежде чем перейти к рассмотрению этих двух типов редукций, напомним, как центральные элементы получаются в случае алгебры $A_{n}$.

8.1. Центральные элементы алгебры $A_{n}$. Соотношение (21) выполнено также и для транспонированной матрицы $\mathscr{A}$ :

$$
\beta_{i, i+1} \mathscr{A}^{T}=B_{i, i+1} \mathscr{A}^{T} B_{i, i+1}^{T}
$$

а следовательно, и для произвольной линейной комбинации $\lambda \mathscr{A}+\lambda^{-1} \mathscr{A}^{T}$, определитель которой, тем самым, оказывается инвариантом относительно действия группы кос [8]. Поскольку $\operatorname{det} \mathscr{A}=1$, производящую функцию для центральных элементов алгебры Пуассона можно представить в виде

$$
\operatorname{det}\left(\mathscr{A}^{-T} \mathscr{A}-\varphi\right)=\operatorname{det}\left(\lambda \mathscr{A}+\lambda^{-1} \mathscr{A}^{T}\right) \lambda^{-n}, \quad \varphi=-\lambda^{2},
$$

и она одновременно является производящей функцией инвариантов группы кос. Рассматривая алгебру Нельсон-Редже (16) как абстрактную алгебру, порождаемую $n(n-1) / 2$ элементами, найдем, что полное число (возможно, независимых) центральных элементов равно $[n / 2]$. 
8.2. Редукции уровня $p$. Редукция уровня $p$ накладывает условие

$$
M_{h}^{p}=\mathbb{E}
$$

при некотором целом $p$. В случае матриц монодромий размера $2 \times 2$ имеем

$$
\operatorname{Tr} M_{h}=2 \cos \left(\frac{2 \pi k}{p}\right)=e^{P_{h} / 2}+e^{-P_{h} / 2}
$$

где $k$ - целое число, а $P_{h}=4 \pi i k / p$ - комплекснозначная длина периметра второй дырки. Условие (65) автоматически оказывается пуассоновым, поскольку его подстановка в соотношения (48) и (49) при $k=p$ приводит к тождествам.

В геометрическом подходе смысл этого условия состоит в том, что вместо новой дырки берется новая орбифолдная точка порядка $p$. На уровне элементов алгебры $\mathfrak{D}_{n}$ получим равенства $G_{i, j}^{(k+p)}=G_{i, j}^{(k)}$, или, поскольку $G_{j, i}^{(-k)}=G_{i, j}^{(k)}$, равенства

$$
G_{i, j}^{(k)}=G_{j, i}^{(p-k)}, \quad k=0, \ldots, p-1
$$
Ввиду этого условия производящая функция переходит в $\mathscr{G}(\lambda)=\frac{1}{\lambda^{p}-1} \mathscr{G}_{p}(\lambda)$,
где

$$
\mathscr{G}_{p}(\lambda):=\mathscr{A}^{(0)}+\frac{\mathscr{G}^{(1)}}{\lambda}+\cdots+\frac{\mathscr{G}^{(p-1)}}{\lambda^{p-1}}+\frac{\left(\mathscr{A}^{(0)}\right)^{T}}{\lambda^{p}},
$$

так что алгебра становится конечно порожденной; будем обозначать ее через $\mathfrak{D}_{n}^{(p)}$.

Соотношения группы кос и центральные элементы.

ПРЕДЛОЖЕНИЕ 8.1. Преобразования группы кос (59) и (60) сохраняют свой матричный вид и при условии редукиии (66), если производится замена $\mathscr{G}(\lambda)$ на $\mathscr{G}_{p}(\lambda)$, заданную формулой (67). В алгебре $\mathfrak{D}_{n}^{(p)}$ имеется ровно $[n p / 2]$ алгебраически независимых иентралъных элементов, порождаемых $\operatorname{det} \mathscr{G}_{p}(\lambda)$.

Доказательство этого утверждения весьма трудоемко, см. приложение С к работе [9].

ЗАмЕчАНИЕ 8.2. Заметим, что при $p=1$ определитель $\operatorname{det} \mathscr{G}_{1}(\lambda)$ переходит в производящую функцию (64) инвариантов группы кос для алгебры $A_{n}$.

Видно, что геометрический случай отвечает сильно вырожденным симплектическим листам, имеющим размерности $2(n-2)$, которые сильно отличаются от максимально возможных размерностей пуассоновых листов, равных $\frac{n^{2} p}{2}-\frac{n p}{2}=\frac{n(n-1) p}{2}$ при четных $p$ и произвольных $n, \frac{n(n-1) p}{2}-\frac{n}{2}$ при нечетных $p$ и четных $n$ и $\frac{n(n-1) p}{2}-\frac{n-1}{2}$ при нечетных $p$ и нечетных $n$. 
8.3. Алгебра $D_{n}$ как редукция алгебры $\mathfrak{D}_{n}$.

8.3.1. Основные соотношения редукции $\mathfrak{D}_{n} \rightarrow D_{n}$. Отождествим на первом этапе те элементы алгебры $\mathfrak{D}_{n}$, которые отвечают геодезическим без самопересечений, с соответствующими элементами алгебры $D_{n}$ (см. рис. 13):

$$
\begin{array}{ll}
G_{i, j}^{(0)} \rightarrow \widehat{G}_{i, j}, & 1 \leqslant i<j \leqslant n, \\
G_{i, j}^{(1)} \rightarrow \widehat{G}_{i, j}, & 1 \leqslant j<i \leqslant n .
\end{array}
$$

Теперь применим распутывающее соотношение, чтобы представить элементы $G_{i, j}^{(1)}$ с $i \leqslant j$ :

$$
\begin{array}{ll}
G_{i, i}^{(1)} \rightarrow \widehat{G}_{i, i}^{2}+\Pi^{2}-2, & 1 \leqslant i \leqslant n, \\
G_{i, j}^{(1)} \rightarrow 2 \widehat{G}_{i, i} \widehat{G}_{j, j}-\widehat{G}_{j, i}+\left(\Pi^{2}-2\right) \widehat{G}_{i, j}, & 1 \leqslant i<j \leqslant n,
\end{array}
$$

или, в графическом виде,

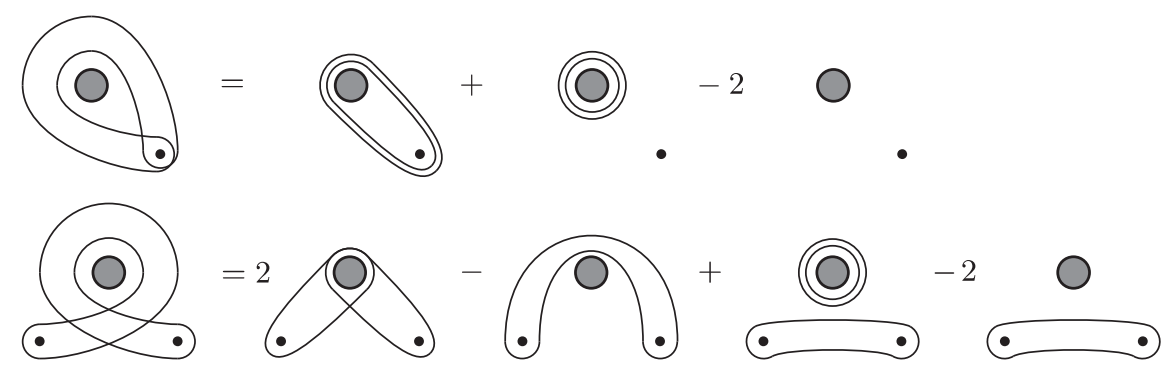

Отметим, во-первых, возникновение параметра

$$
\Pi:=e^{P_{h} / 2}+e^{-P_{h} / 2},
$$

являющегося геодезической функцией для второй дырки с длиной периметра $P_{h}$, и, во-вторых, возможность переписать соотношение (69) в виде 8

$$
G_{i, j}^{(1)} \rightarrow 2 \widehat{G}_{i, i} \widehat{G}_{j, j}-G_{j, i}^{(1)}+\left(\Pi^{2}-2\right) G_{i, j}^{(0)}, \quad 1 \leqslant i<j \leqslant n,
$$

или, с учетом того, что $\mathscr{G}^{(k)}=(\mathscr{G}(-k))^{T}$, в виде

$$
G_{i, j}^{(1)} \rightarrow 2 \widehat{G}_{i, i} \widehat{G}_{j, j}-G_{i, j}^{(-1)}+\left(\Pi^{2}-2\right) G_{i, j}^{(0)}, \quad 1 \leqslant i<j \leqslant n, \quad \Pi \neq 0 .
$$

Оказывается особенно полезным представить это соотношение редукции в терминах матриц $\widehat{\mathscr{A}}, \widehat{\mathscr{R}}$ и $\widehat{\mathscr{S}}$, задаваемых соответствующими выражениями (29), (27) и (28):

$$
\mathscr{G}^{(1)} \rightarrow \widehat{\mathscr{R}}+\widehat{\mathscr{S}}+\left(\Pi^{2}-1\right) \widehat{\mathscr{A}}-\widehat{\mathscr{A}}^{T}
$$

8Заметим, что все элементы $\widehat{G}_{i, j}$ при $i \neq j$ выражаются в виде многочленов от элементов $G_{a, b}$ алгебры $A_{n+m}$; это свойство, однако, не выполнено для диагональных элементов $\widehat{G}_{i, i}$, которые не представимы в виде полиномиальных функций от генераторов алгебры $A_{n+m}$. 
Продолжим выражать элементы старших уровней $\mathscr{G}^{(k)}$, фиксируя индекс $i$ и вращая конец с индексом $j$ в направлении против часовой стрелки, как показано ниже (продолжая вращать конец $j$, получим в левой части геодезическую, отвечающую двукратному обходу вокруг дырки):

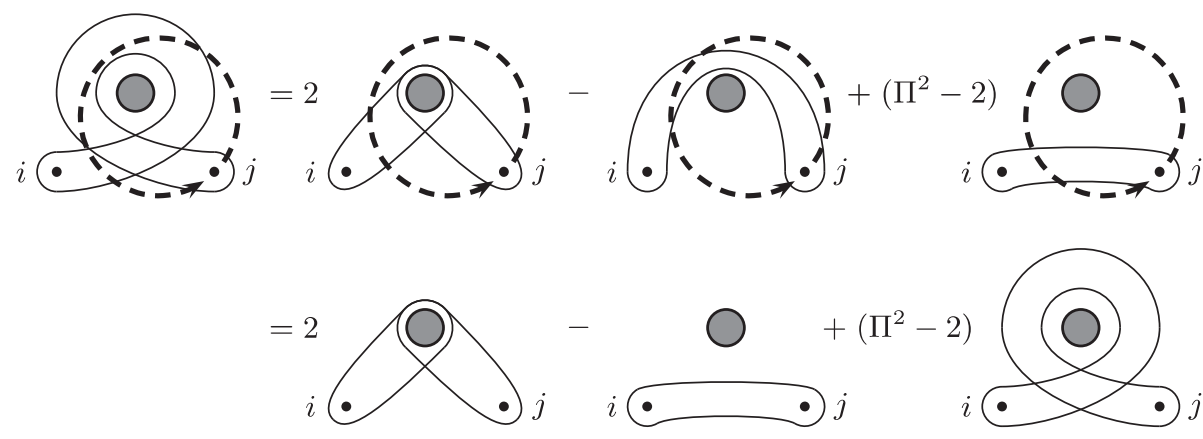

По совершении полного оборота конца с индексом $j$ против часовой стрелки, получим в левой части элемент $G_{i, j}^{(2)}$, в то время как в правой части произведение $\widehat{G}_{i, i} \widehat{G}_{j, j}$ оказывается циклически инвариантным, член $-G_{i, j}^{(-1)}$ перейдет в $-G_{i, j}^{(0)}=-\widehat{G}_{i, j}$, а последнее слагаемое $\left(\Pi^{2}-2\right) G_{i, j}^{(0)}$ превратится в $\left(\Pi^{2}-2\right) G_{i, j}^{(1)}$, после чего к нему следует снова применить условие редукции (70) и представить матрицу $\mathscr{G}^{(2)}$ в виде линейной комбинации матриц $\widehat{\mathscr{R}}, \widehat{\mathscr{S}}, \widehat{\mathscr{A}}$ и $\widehat{\mathscr{A}}^{T}$. Повторяя этот процесс, придем к следующему закону редукции для $\mathscr{G}^{(k)}$ :

$$
\begin{aligned}
& \mathscr{G}^{(k)} \rightarrow \frac{e^{k P_{h}}-e^{-k P_{h}}}{e^{P_{h}}-e^{-P_{h}}} \widehat{\mathscr{R}}+\frac{e^{k P_{h}}-2+e^{-k P_{h}}}{\left(e^{P_{h}}-1\right)\left(1-e^{-P_{h}}\right)} \hat{\mathscr{S}}+\left[\frac{e^{k P_{h}}}{1-e^{-P_{h}}}\right. \\
&\left.-\frac{e^{-k P_{h}}}{e^{P_{h}}-1}\right] \widehat{\mathscr{A}}-\left[\frac{e^{(k-1) P_{h}}}{1-e^{-P_{h}}}-\frac{e^{-(k-1) P_{h}}}{e^{P_{h}}-1}\right] \widehat{\mathscr{A}}^{T}, \quad k \geqslant 1 .
\end{aligned}
$$

ТЕОрема 8.3 [9]. Выражения (59) и (60) задают представление действия группы кос $(24),(25)$ на элементы алгебры $D_{n}$ при произвольном ненулевом П при условии, что матрицы $\mathscr{G}^{(k)}$ выражаются через элементы $\widehat{G}_{i, j}$ и параметр $\Pi \neq 0$ с помощъю формул (71).

Заметим, что одно из наиболее интересных свойств соотношений группы кос как в случае алгебры $\mathfrak{D}_{n}$, так и в случае алгебры $D_{n}$ состоит в том, что ни одно из этих соотношений не содержит зависимости от длин периметров дырок $P$ и $P_{h}$. Таким образом, ввиду того, что оба эти набора соотношений задают одни и те же преобразования группы классов отображений в геометрическом подходе, естественно ожидать, что они также будут совпадать, если применить процедуру редукции, основанную на распутывающем соотношении. Это приводит к следующему выводу.

СлеДСтвиЕ 8.4. Редукиия уровня р согласуется с редукиией $D_{n}$ при условии $\left(e^{P_{h}}\right)^{p}=1$. 
8.3.2. Центральные элементы алгебры $D_{n}$. Подставим теперь формулы редукции (71) в представление (45) и явно произведем суммирование по степеням $\lambda^{-1}$. В результате получим

$$
\begin{aligned}
\mathscr{G}(\lambda) \rightarrow & \frac{\lambda}{(\lambda-1)\left(e^{-P_{h}} \lambda-1\right)\left(e^{P_{h}} \lambda-1\right)} \\
& \times\left[(\lambda-1) \widehat{\mathscr{R}}+(\lambda+1) \widehat{\mathscr{S}}+\left(\lambda^{2}-1\right) \widehat{\mathscr{A}}-\left(\lambda-\lambda^{-1}\right) \widehat{\mathscr{A}}^{T}\right],
\end{aligned}
$$

что приводит к следующему утверждению.

ПреДЛОЖЕНИЕ 8.5. Алгебра $D_{n}$ имеет в точности $n$ алгебраически независимых иентральных элементов $c_{1}, \ldots, c_{n}$. Они задаются производящей функцией

$$
\begin{aligned}
\operatorname{det}[ & \left.(\lambda-1) \widehat{\mathscr{R}}+(\lambda+1) \widehat{\mathscr{S}}+\left(\lambda^{2}-1\right) \widehat{\mathscr{A}}-\left(\lambda-\lambda^{-1}\right) \widehat{\mathscr{A}}^{T}\right] \\
& =(\lambda-1)^{n-1}\left[\lambda^{n+1}+\sum_{i=1}^{n} \lambda^{i} c_{i}+(-1)^{n+1} \sum_{i=1}^{n} \lambda^{1-i} c_{i}+(-1)^{n+1} \lambda^{-n}\right] .
\end{aligned}
$$

КОММЕНТАРИИ К ДОКАЗАТЕЛЬСТВУ. То, что центральные элементы задаются определителем $\operatorname{det} \mathscr{G}(\lambda)$ следует из теоремы 8.3 и из формулы для центральных элементов алгебры $\mathfrak{D}_{n}$. То, что этот определитель принимает вид, указанный во второй строке выражения (73), следует из подстановки (71) и того факта, что матрица $\widehat{\mathscr{S}}$ имеет единичный ранг, а потому во всех произведениях элементов, входящих в разложение определителя полной матрицы, может участвовать не более одного элемента матрицы $\widehat{\mathscr{S}}$, в то время как все остальные элементы содержат множитель $(\lambda-1)$. Алгебраическая независимость элементов $c_{1}, \ldots, c_{n}$ была доказана в работе [9] с помощью выбора специальной точки, в которой $\widehat{G}_{i, j}=0$ при $i \neq j, \widehat{G}_{i, i} \neq 0$ и $\widehat{G}_{i, i}^{2} \neq \widehat{G}_{j, j}^{2}$ при $i \neq j$.

8.3.3. Центральные элементы алгебр $D_{2}$ и $D_{3}$. В случае алгебры $D_{2}$ имеются следующие два центральных элемента:

$$
\begin{aligned}
& C_{1}^{(2)}=\widehat{G}_{1,1} \widehat{G}_{2,2}-\widehat{G}_{1,2}-\widehat{G}_{2,1}, \\
& C_{2}^{(2)}=\widehat{G}_{1,2} \widehat{G}_{2,1}-\widehat{G}_{1,1}^{2}-\widehat{G}_{2,2}^{2} .
\end{aligned}
$$

В случае алгебры $D_{3}$ имеем три центральных элемента:

$$
\begin{aligned}
& C_{1}^{(3)}=\widehat{G}_{1,1} \widehat{G}_{2,2} \widehat{G}_{3,3}-\widehat{G}_{1,1}\left(\widehat{G}_{3,2}+\widehat{G}_{2,3}\right)-\widehat{G}_{2,2}\left(\widehat{G}_{1,3}+\widehat{G}_{3,1}\right)-\widehat{G}_{3,3}\left(\widehat{G}_{2,1}+\widehat{G}_{1,2}\right), \\
& C_{2}^{(3)}=\widehat{G}_{1,2} \widehat{G}_{2,3} \widehat{G}_{3,1}-\widehat{G}_{1,2} \widehat{G}_{2,1}-\widehat{G}_{2,3} \widehat{G}_{3,2}-\widehat{G}_{3,1} \widehat{G}_{1,3}+\widehat{G}_{1,1}^{2}+\widehat{G}_{2,2}^{2}+\widehat{G}_{3,3}^{2}, \\
& C_{3}^{(3)=} \widehat{G}_{1,3} \widehat{G}_{2,1} \widehat{G}_{3,2}-\widehat{G}_{1,2} \widehat{G}_{2,1} \widehat{G}_{3,3}^{2}-\widehat{G}_{2,3} \widehat{G}_{3,2} \widehat{G}_{1,1}^{2}-\widehat{G}_{3,1} \widehat{G}_{1,3} \widehat{G}_{2,2}^{2} \\
&+ 2 \widehat{G}_{1,1} \widehat{G}_{2,2}\left(\widehat{G}_{2,3} \widehat{G}_{3,1}-\widehat{G}_{2,1}-\widehat{G}_{1,2}\right) \\
&+2 \widehat{G}_{2,2} \widehat{G}_{3,3}\left(\widehat{G}_{3,1} \widehat{G}_{1,2}-\widehat{G}_{3,2}-\widehat{G}_{2,3}\right) \\
&+2 \widehat{G}_{3,3} \widehat{G}_{1,1}\left(\widehat{G}_{3,1} \widehat{G}_{1,2}-\widehat{G}_{3,2}-\widehat{G}_{2,3}\right)+\widehat{G}_{2,1}^{2}+\widehat{G}_{3,2}^{2}+\widehat{G}_{1,3}^{2} \\
&-\widehat{G}_{1,2} \widehat{G}_{2,3} \widehat{G}_{1,3}-\widehat{G}_{2,3} \widehat{G}_{3,1} \widehat{G}_{2,1}-\widehat{G}_{3,1} \widehat{G}_{1,2} \widehat{G}_{3,2}+\widehat{G}_{1,2}^{2}+\widehat{G}_{2,3}^{2}+\widehat{G}_{3,1}^{2} \\
&+\left(\widehat{G}_{1,1}^{2}+1\right)\left(\widehat{G}_{2,2}^{2}+1\right)+\left(\widehat{G}_{2,2}^{2}+1\right)\left(\widehat{G}_{3,3}^{2}+1\right)+\left(\widehat{G}_{3,3}^{2}+1\right)\left(\widehat{G}_{1,1}^{2}+1\right) .
\end{aligned}
$$




\section{9. Квантовые пространства Тейхмюллера орбифолдных римановых поверхностей}

9.1. Каноническое квантование алгебр Пуассона. Квантование пуассонова многообразия, эквивариантное относительно действия дискретной группы $\mathscr{D}$, задается семейством *-алгебр $\mathscr{A}^{\hbar}$, зависящих от вещественного параметра $\hbar$, с действием $\mathscr{D}$ с помощью внешних автоморфизмов.

Зафиксируем остовный граф поверхности $\Sigma_{g, s, \delta}$ и обозначим через $\mathscr{T}^{\hbar}:=$ $\mathscr{T}^{\hbar}\left(\Gamma_{g, s,\left|\delta^{(1)}\right|,\left|\delta^{(2)}\right|}\right)$ алгебру, порождаемую операторами $Z_{\alpha}^{\hbar}$, по одному генератору на каждое неориентированное ребро $\alpha$ графа $\Gamma_{g, s,\left|\delta^{(1)}\right|,\left|\delta^{(2)}\right|}$, с соотношениями

$$
\left[Z_{\alpha}^{\hbar}, Z_{\beta}^{\hbar}\right]=2 \pi i \hbar\left\{Z_{\alpha}, Z_{\beta}\right\}
$$

(ср. (7)) и с $*$-структурой (эрмитовым сопряжением)

$$
\left(Z_{\alpha}^{\hbar}\right)^{*}=Z_{\alpha}^{\hbar}
$$

где через $Z_{\alpha}$ и $\{\cdot, \cdot\}$ обозначены соответственно координатные функции и пуассоновы скобки на классическом пространстве Тейхмюллера. Правая часть соотношений (74) равна константе.

Все стандартные утверждения, имеющие место в случае пространств Тейхмюллера римановых поверхностей с дырками, остаются справедливыми и в случае орбифолдных римановых поверхностей.

Лемма 9.1. Центр $\mathscr{Z}^{\hbar}$ алгебры $\mathscr{T}^{\hbar}$ порождается суммами $\sum_{\alpha \in I} Z_{\alpha}^{\hbar}$, берущимися по всем ребрам $\alpha \in I$, принадлежащим данной граничной компоненте; размерность иентра равна $s$, и квантовая структура невырождена на факторпространстве $\mathscr{T}^{\hbar} / \mathscr{Z}^{\hbar}$.

9.2. Квантовые геодезические функции. Вложим теперь алгебру геодезических функций (4) в подходящее замыкание уже построенной алгебры $\mathscr{T}^{\hbar}$. Для произвольной геодезической $\gamma$ ее геодезическая функция $G_{\gamma}$ выражается через координаты сдвигов:

$$
G_{\gamma} \equiv \operatorname{Tr} P_{Z_{1} \cdots Z_{n}}=\sum_{j \in J} \exp \left\{\frac{1}{2} \sum_{\alpha \in E(\Gamma)} m_{j}(\gamma, \alpha) Z_{\alpha}\right\}
$$

где $m_{j}(\gamma, \alpha)$ - целые числа, $E(\Gamma)$ - множество ненаправленных ребер графа $\Gamma$, а $J$ - конечный набор индексов.

В общем случае наборы индексов $\left\{m_{j}(\gamma, \alpha)\right\}_{\alpha=1}^{6 g-6+3 s+2|\delta|}$ могут совпадать при разных $j_{1}, j_{2} \in J$; будем тем не менее различать эти наборы, так как они приходят из разных произведений экспоненциированных членов $e^{ \pm Z_{i} / 2}$ в следах матричных произведений в (76).

Для произвольного замкнутого пути $\gamma$ на $\Sigma_{g, s, \delta}$ определим оператор квантовой геодезической функиии $G_{\gamma}^{\hbar} \in \mathscr{T}^{\hbar}$ формулой

$$
G_{\gamma}^{\hbar} \equiv \underset{\times}{\times} \operatorname{Tr} P_{Z_{1} \cdots Z_{n}} \stackrel{\times}{\times} \equiv \sum_{j \in J} \exp \left\{\frac{1}{2} \sum_{\alpha \in E\left(\Gamma_{g, \delta}\right)}\left(m_{j}(\gamma, \alpha) Z_{\alpha}^{\hbar}+2 \pi i \hbar c_{j}(\gamma, \alpha)\right)\right\}
$$


где символ квантового упорядочения $\underset{\times}{\times} \cdot \underset{\times}{\times}$ предполагает, что классическое выражение (76) должно быть подправлено: в него вводятся дополнительные целочисленные коэффициенты $c_{j}(\gamma, \alpha)$, явный вид которых следует из нижеприведенных условий 1-5. Тем самым предполагается, что каждый член классического разложения (76) может быть подправлен максимум на дополнительный множитель вида $q^{n}, n \in \mathbb{Z}, \mathrm{c}$

$$
q \equiv e^{-i \pi \hbar}
$$

Определяющие свойства квантовых геодезических функций следующие:

1. Коммутативность. Если замкнутые пути $\gamma$ и $\gamma^{\prime}$ не пересекаются, то операторы $G_{\gamma}^{\hbar}$ и $G_{\gamma^{\prime}}^{\hbar}$ коммутируют. Из этого, в частности, следует, что определено понятие квантовой геодезической ламинации, $Q G M:=$ $\prod_{\gamma \in \Gamma л}\left(G_{\gamma}^{\hbar}\right)^{m_{\gamma}}$, поскольку множители в этом произведении взаимно коммутируют.

2. Eстественностъ. Группа классов отображений (8) действует естественным образом на множестве $\left\{G_{\gamma}^{\hbar}\right\}$, т. е. для каждого преобразования $W^{\hbar}$ из группоида классов отображений и для произвольного замкнутого пути $\gamma$ на остовном графе $\Gamma_{g, s,\left|\delta^{(1)}\right|,\left|\delta^{(2)}\right|}$ римановой поверхности $\Sigma_{g, s, \delta}$ имеем

$$
W^{\hbar}\left(G_{\gamma}^{\hbar}\right)=G_{W(\gamma)}^{\hbar}
$$

3. Алгебра квантовых геодезических функиий. Произведение двух квантовых геодезических функций задается линейной комбинацией квантовых геодезических ламинаций, подчиняющихся (квантовым) распутывающим соотношениям, которые приведены в формуле (79).

4. Независимость от ориентации. Как и в классическом случае, квантовые геодезические функции не зависят от направления обхода вокруг соответствующей геодезической кривой.

5. Степени геодезических. Квантовая геодезическая функция $G_{n \gamma}^{\hbar}$, отвечающая $n$-кратному обходу вокруг геодезической кривой $\gamma$, выражается через $G_{\gamma}^{\hbar}$ в точности так же, как и в классическом случае, а именно,

$$
G_{n \gamma}^{\hbar}=2 T_{n}\left(\frac{G_{\gamma}^{\hbar}}{2}\right)
$$

где $T_{n}(x)$ - многочлены Чебышёва.

6. Эрмитовость. Все квантовые геодезические функции суть эрмитовы операторы, имеющие по определению вещественный спектр.

9.3. Квантовые распутывающие соотношения. Сформулируем теперь общие правила, позволяющие распутать произведение любых двух квантовых геодезических функций.

Пусть $G_{1}^{\hbar}$ и $G_{2}^{\hbar}$ - две квантовые геодезические функции, отвечающие геодезическим $\gamma_{1}$ и $\gamma_{2}$. Тогда: 
- следует применить квантовое распутывающее соотношение ${ }^{9}$

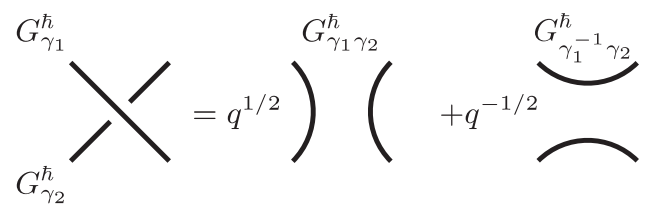

одновременно во всех точках пересечения;

- после применения квантового распутывающего соотношения может получиться пустая (стягиваемая) петля; такой петле соответствует множитель $-q-q^{-1}$, что достаточно для того, чтобы обеспечить выполнение квантовых движений Рейдемейстера;

- могут также получиться петли, гомеоморфные обходам вокруг точечных или звездчатых вершин; как и в классическом случае, будем считать, что соответствующая квантовая геодезическая функция зануляется в случае точечной вершины, $\operatorname{Tr} F=0$, что зануляет всю соответствующую квантовую геодезическую ламинацию, а в случае звездчатой вершины она по-прежнему равна единице (не получает квантовых поправок).

Лемма 9.2 [25], [26], [33]. Имеется единственное квантовое упорядочение $\times$ ×..× $\times$ (77), которое задается алгеброй квантовых геодезических функций (79) и согласовано с преобразованиями из квантовой группы классов отображсений.

Пример 9.3. Для картины квантовых геодезических функций на рис. 11, в случае точечной вершины, имеем (при условии, что геодезические $\gamma_{1}$ и $\gamma_{2}$ не имеют других точек пересечения)

$$
G_{1}^{\hbar} \cdot G_{2}^{\hbar}=q G_{\cup}^{\hbar}+q^{-1} G_{\cap}^{\hbar}+G_{\supset}^{\hbar} \cdot G_{\subset}^{\hbar} .
$$

Эта алгебра упрощается еще больше в случае, когда $G_{1}^{\hbar}={ }_{\times}^{\times} \operatorname{Tr} F_{i_{1}} F_{j} \times{ }_{\times}:=G_{i_{1}, j}^{\hbar}$ и $G_{2}^{\hbar}={ }_{\times}^{\times} \operatorname{Tr} F_{j} F_{i_{2}} \times{ }_{\times}:=G_{j, i_{2}}^{\hbar}$ с $i_{1}<j<i_{2}$, поскольку в этом случае $G_{\supset}^{\hbar} \stackrel{i_{1}, j}{=}$

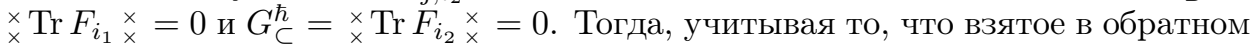
порядке произведение дает

$$
G_{2}^{\hbar} \cdot G_{1}^{\hbar}=q^{-1} G_{\cup}^{\hbar}+q G_{\cap}^{\hbar}+G_{\supset}^{\hbar} \cdot G_{\subset}^{\hbar},
$$

и то, что при $G_{1}^{\hbar}=G_{i_{1}, j}^{\hbar}$ и $G_{2}^{\hbar}=G_{j, i_{2}}^{\hbar}$ выполнено равенство $G_{\cap}^{\hbar}={ }_{\times}^{\times} \operatorname{Tr} F_{i_{1}} F_{i_{2}} \times{ }_{\times}:=$ $G_{i_{1}, i_{2}}^{\hbar}$, мы приходим к $q$-коммутачионному соотношению

$$
q G_{i_{1}, j}^{\hbar} \cdot G_{j, i_{2}}^{\hbar}-q^{-1} G_{j, i_{2}}^{\hbar} \cdot G_{i_{1}, j}^{\hbar}=\left(q^{2}-q^{-2}\right) G_{i_{1}, i_{2}}^{\hbar} \cdot
$$

Это соотношение числится среди основных соотношений квантовых алгебр Нельсон-Редже [2].

9.4. Квантовая алгебра $A_{n}$. Рассмотрим теперь квантовые геодезические функции, отвечающие путям в картине алгебры $A_{n}$, представленной на рис. 12 .

\footnotetext{
${ }^{9}$ Порядок пересечения линий зависит от того, какая квантовая геодезическая функция стоит на первом месте в произведении.
} 
Обобщим сначала пример 9.3 на случай общей алгебры $A_{n}$. Для квантовых геодезических функций $G_{i, j}^{\hbar}(i<j)$ имеем (в предположении, что $\left.j<i<l<k\right)$ :

$$
\begin{gathered}
{\left[G_{i, k}^{\hbar}, G_{j, l}^{\hbar}\right]=\xi\left(G_{j, k}^{\hbar} G_{i, l}^{\hbar}-G_{j, i}^{\hbar} G_{l, k}^{\hbar}\right),} \\
q G_{i, l}^{\hbar} G_{j, i}^{\hbar}-q^{-1} G_{j, i}^{\hbar} G_{i, l}^{\hbar}=\xi G_{j, l}^{\hbar}, \quad \xi=q^{2}-q^{-2}, \\
q G_{j, l}^{\hbar} G_{i, l}^{\hbar}-q^{-1} G_{i, l}^{\hbar} G_{j, l}^{\hbar}=\xi G_{j, i}^{\hbar},
\end{gathered}
$$

и, разумеется, квантовые геодезические функции, отвечающие непересекающимся геодезическим, взаимно коммутируют.

С учетом квантового соотношения распутывания можно довольно легко получить квантовые преобразования для $G_{i, j}^{\hbar}$. Введем квантовую $\mathscr{A}^{\hbar}$-матрицу

$$
\mathscr{A}^{\hbar}=\left(\begin{array}{ccccc}
q^{-1} & G_{1,2}^{\hbar} & G_{1,3}^{\hbar} & \ldots & G_{1, n}^{\hbar} \\
0 & q^{-1} & G_{2,3}^{\hbar} & \ldots & G_{2, n}^{\hbar} \\
0 & 0 & q^{-1} & \ddots & \vdots \\
\vdots & \vdots & \ddots & \ddots & G_{n-1, n}^{\hbar} \\
0 & 0 & \ldots & 0 & q^{-1}
\end{array}\right),
$$

в которой эрмитовы операторы $G_{i, j}^{\hbar}$ отождествляются с квантовыми геодезическими функциями. Применяя квантовое соотношение распутывания, можно выразить действие элемента группы кос $\beta_{i, i+1}^{\hbar}$ в терминах квантовых геодезических функций исключительно из этого фиксированного набора, $\beta_{i, i+1}^{\hbar} \mathscr{A}^{\hbar}=\tilde{\mathscr{A}}^{\hbar}$, где

$$
\begin{aligned}
\widetilde{G}_{i+1, j}^{\hbar} & =G_{i, j}^{\hbar}, & & j>i+1, \\
\widetilde{G}_{j, i+1}^{\hbar} & =G_{j, i}^{\hbar}, & & j<i, \\
\widetilde{G}_{i, j}^{\hbar} & =q^{-1} G_{i, j}^{\hbar} G_{i, i+1}^{\hbar}-q^{-2} G_{i+1, j}^{\hbar}=q G_{i, i+1}^{\hbar} G_{i, j}^{\hbar}-q^{2} G_{i+1, j}^{\hbar}, & & j>i+1, \\
\widetilde{G}_{j, i}^{\hbar} & =q^{-1} G_{j, i}^{\hbar} G_{i, i+1}^{\hbar}-q^{-2} G_{j, i+1}^{\hbar}=q G_{i, i+1}^{\hbar} G_{j, i}^{\hbar}-q^{2} G_{j, i+1}^{\hbar}, & & j<i, \\
\widetilde{G}_{i, i+1}^{\hbar} & =G_{i, i+1}^{\hbar} . & &
\end{aligned}
$$

Применяя специальные матрицы $B_{i, i+1}^{\hbar}$ блочно-диагонального вида

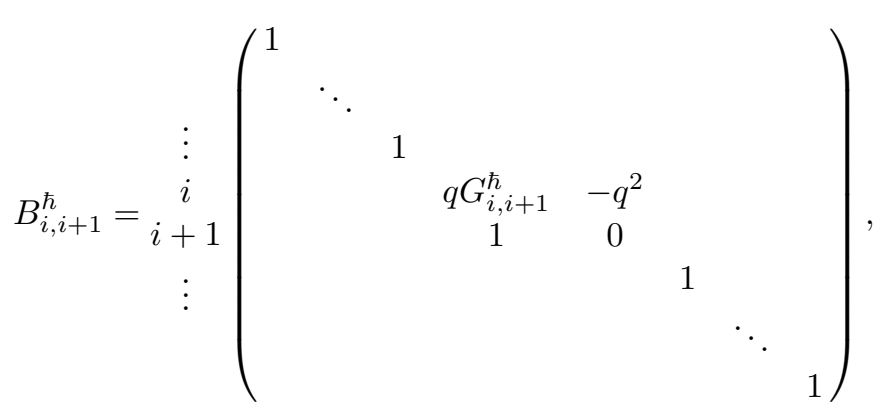

можно снова представить преобразования квантовой группы кос (82) в виде матричного произведения (с учетом некоммутативности элементов квантовых матриц):

$$
\beta_{i, i+1}^{\hbar} \mathscr{A}^{\hbar}=B_{i, i+1}^{\hbar} \mathscr{A}^{\hbar}\left(B_{i, i+1}^{\hbar}\right)^{\dagger},
$$


где $\left(B_{i, i+1}^{\hbar}\right)^{\dagger}$ - матрица, эрмитово сопряженная матрице $B_{i, i+1}^{\hbar}$ (ее нетривиальный $(2 \times 2)$-блок имеет вид $\left.\left(\begin{array}{cc}q^{-1} G_{i, i+1}^{\hbar} & 1 \\ -q^{-2} & 0\end{array}\right)\right)$. Применяя те же методы, что и раньше, можно доказать следующую лемму.

ЛЕмма 9.4. При любом $n \geqslant 3$ имеют место соотношения квантовой групnы кос

$$
\begin{gathered}
\beta_{i-1, i}^{\hbar} \beta_{i, i+1}^{\hbar} \beta_{i-1, i}^{\hbar}=\beta_{i, i+1}^{\hbar} \beta_{i-1, i}^{\hbar} \beta_{i, i+1}^{\hbar}, \quad 2 \leqslant i \leqslant n-1, \\
\left(\beta_{n-1, n}^{\hbar} \beta_{n-2, n-1}^{\hbar} \cdots \beta_{2,3}^{\hbar} \beta_{1,2}^{\hbar}\right)^{n}=\mathbb{E} .
\end{gathered}
$$

9.5. Действие квантовой группы кос на твистованном янгиане. Квантовые геодезические функции $G_{i, j}^{(k)^{\hbar}}$ суть эрмитовы операторы, подчиняющиеся квантовым перестановочным соотношениям (53). Действие группы кос при этом получается из соответствующего действия для квантовой алгебры $D_{n}$, построенного в работе [6].

Определим квантовый оператор $\mathscr{G}^{\hbar}(\lambda)$ следующего вида:

$$
\mathscr{G}_{i, j}^{\hbar}(\lambda):=\mathscr{A}_{i, j}^{\hbar}+\sum_{k=1}^{\infty} G_{i, j}^{(k)^{\hbar}} \lambda^{-k},
$$

где $\mathscr{A}^{\hbar}-$ верхнетреугольная матрица с элементами

$$
\mathscr{A}_{i, j}^{\hbar}= \begin{cases}G_{i, j}^{(0)}, & i<j \\ q^{-1}, & i=j, \\ 0, & i>j\end{cases}
$$

(cp. (81)). Напомним, что $q^{\dagger}=q^{-1}$.

ПРеДЛОЖеНИЕ 9.5. Преобразования группъ кос для квантовой алгебры $\mathfrak{D}_{n}$ могут быть представлены в следующем матричном виде в терминах матрицы $\mathscr{G}^{\hbar}(\lambda)(85)$ :

$$
\beta_{i, i+1}^{\hbar} \mathscr{G}^{\hbar}(\lambda)=B_{i, i+1}^{\hbar} \mathscr{G}^{\hbar}(\lambda)\left(B_{i, i+1}^{\hbar}\right)^{\dagger}, \quad i=1, \ldots, n-1,
$$

где матрица $B_{i, i+1}^{\hbar}$ имеет вид (83), в котором оператор $G_{i, i+1}^{\hbar}$ следует заменить на оператор $G_{i, i+1}^{(0)}{ }^{\hbar}$, а действие генератора $\beta_{n, 1}^{\hbar}$ записъвается в виде

$$
\beta_{n, 1}^{\hbar} \mathscr{G}^{\hbar}(\lambda)=B_{n, 1}^{\hbar}(\lambda) \mathscr{G}^{\hbar}(\lambda)\left(B_{n, 1}^{\hbar}\left(\lambda^{-1}\right)\right)^{\dagger}
$$

¿əe

$$
B_{n, 1}^{\hbar}(\lambda)=\left(\begin{array}{ccccc}
0 & 0 & \ldots & 0 & \lambda \\
0 & 1 & 0 & \ldots & 0 \\
\vdots & 0 & \ddots & \ddots & \vdots \\
0 & \vdots & \ddots & 1 & 0 \\
-q^{2} \lambda^{-1} & 0 & \ldots & 0 & q G_{n, 1}^{(1)}
\end{array}\right)
$$

9.6. Квантовая алгебра $D_{n}$. Приведем теперь квантовую версию пуассоновых соотношений для геодезических функций $G_{i, j}$, отвечающих путям на рис. 13. Каждому пуассонову коммутатору генераторов алгебры $D_{n}$ из п. 7.3 поставим в соответствие квантовое перестановочное соотношение. 
В нижеприведенных формулах будем предполагать индексы $\{i, l, k, j\}$ циклически упорядоченными. Тогда квантовые перестановочные соотношения имеют следующий вид $\left(q=e^{-i \pi \hbar}, \xi \equiv q^{2}-q^{-2}\right)$ :

$$
\begin{aligned}
& {\left[\widehat{G}_{i, k}^{\hbar}, \widehat{G}_{j, l}^{\hbar}\right]=\xi\left(\widehat{G}_{j, k}^{\hbar} \widehat{G}_{i, l}^{\hbar}-\widehat{G}_{j, i}^{\hbar} \widehat{G}_{l, k}^{\hbar}\right) \text {; }} \\
& q \widehat{G}_{j, l}^{\hbar} \widehat{G}_{k, j}^{\hbar}-q^{-1} \widehat{G}_{k, j}^{\hbar} \widehat{G}_{j, l}^{\hbar}=\xi \widehat{G}_{k, l}^{\hbar} \text {; } \\
& {\left[\widehat{G}_{j, l}^{\hbar}, \widehat{G}_{i, i}^{\hbar}\right]=\xi\left(\widehat{G}_{j, i}^{\hbar} \widehat{G}_{l, l}^{\hbar}-\widehat{G}_{i, l}^{\hbar} \widehat{G}_{j, j}^{\hbar}\right) \text {; }} \\
& q \widehat{G}_{j, j}^{\hbar} \widehat{G}_{k, j}^{\hbar}-q^{-1} \widehat{G}_{k, j}^{\hbar} \widehat{G}_{j, j}^{\hbar}=\xi \widehat{G}_{k, k}^{\hbar}, \quad q \widehat{G}_{j, k}^{\hbar} \widehat{G}_{j, j}^{\hbar}-q^{-1} \widehat{G}_{j, j}^{\hbar} \widehat{G}_{j, k}^{\hbar} \xi \widehat{G}_{k, k}^{\hbar} ; \\
& {\left[\widehat{G}_{i, i}^{\hbar}, \widehat{G}_{k, k}^{\hbar}\right]=\left(q-q^{-1}\right)\left(\widehat{G}_{i, k}^{\hbar}-\widehat{G}_{k, i}^{\hbar}\right) \text {; }} \\
& {\left[\widehat{G}_{i, j}^{\hbar}, \widehat{G}_{k, l}^{\hbar}\right]=\xi\left(\widehat{G}_{k, j}^{\hbar} \widehat{G}_{l, i}^{\hbar}-\widehat{G}_{j, k}^{\hbar} \widehat{G}_{i, l}^{\hbar}+\widehat{G}_{j, l}^{\hbar} \widehat{G}_{i, k}^{\hbar}-\widehat{G}_{l, j}^{\hbar} \widehat{G}_{k, i}^{\hbar}\right.} \\
& \left.+\left(q+q^{-1}\right)\left(\widehat{G}_{i, l}^{\hbar} \widehat{G}_{j, j}^{\hbar} \widehat{G}_{k, k}^{\hbar}-\widehat{G}_{k, j}^{\hbar} \widehat{G}_{l, l}^{\hbar} \widehat{G}_{i, i}^{\hbar}\right)\right) ; \\
& q \widehat{G}_{j, l}^{\hbar} \widehat{G}_{i, j}^{\hbar}-q^{-1} \widehat{G}_{i, j}^{\hbar} \widehat{G}_{j, l}^{\hbar}=\xi\left(q^{-1} \widehat{G}_{l, j}^{\hbar} \widehat{G}_{j, i}^{\hbar}+q \widehat{G}_{i, i}^{\hbar} \widehat{G}_{l, l}^{\hbar}+q^{-1} \widehat{G}_{l, l}^{\hbar} \widehat{G}_{i, i}^{\hbar}\right. \\
& \left.-q^{-2} \widehat{G}_{l, i}^{\hbar}-\widehat{G}_{i, l}^{\hbar}\left(\widehat{G}_{j, j}^{\hbar}\right)^{2}\right) \text {; } \\
& {\left[\widehat{G}_{j, l}^{\hbar}, \widehat{G}_{l, j}^{\hbar}\right]=\xi\left(\left(\widehat{G}_{l, l}^{\hbar}\right)^{2}-\left(\widehat{G}_{j, j}^{\hbar}\right)^{2}\right) .}
\end{aligned}
$$

Хотя в этих соотношениях встречаются члены третьего порядка и некоммутирующие сомножители в правых частях (это - цена, заплаченная за замыкание алгебры), этих соотношений тем не менее достаточно для того, чтобы установить лексикографическое упорядочение на соответствующем множестве квантовых переменных $\left\{G_{i, j}^{\hbar}\right\}$.

Лемма 9.6. Перестановочные соотношения, устанавливаемые формулами (89), удовлетворяют (квантовым) тождествам Якоби.

Представим теперь квантовую версию преобразований группы кос (24) и (25). Для $\beta_{i, i+1}^{\hbar}$ с $1 \leqslant i \leqslant n-1$ имеем:

$$
\begin{array}{rlr}
\widetilde{\widehat{G}}_{i+1, k}^{\hbar} & =\widehat{G}_{i, k}^{\hbar}, & k \neq i, i+1, \\
\widetilde{\widehat{G}}_{i, k}^{\hbar} & =q \widehat{G}_{i, i+1}^{\hbar} \widehat{G}_{i, k}^{\hbar}-q^{2} \widehat{G}_{i+1, k}^{\hbar}=q^{-1} \widehat{G}_{i, k}^{\hbar} \widehat{G}_{i, i+1}^{\hbar}-q^{-2} \widehat{G}_{i+1, k}^{\hbar}, & k \neq i, i+1, \\
\widetilde{\widehat{G}}_{k, i+1}^{\hbar} & =\widehat{G}_{k, i}^{\hbar}, & k \neq i, i+1, \\
\widetilde{\widehat{G}}_{k, i}^{\hbar} & =q \widehat{G}_{i, i+1}^{\hbar} \widehat{G}_{k, i}^{\hbar}-q^{2} \widehat{G}_{k, i+1}^{\hbar}=q^{-1} \widehat{G}_{k, i}^{\hbar} \widehat{G}_{i, i+1}^{\hbar}-q^{-2} \widehat{G}_{k, i+1}^{\hbar}, & k \neq i, i+1, \\
\widetilde{\widehat{G}}_{i, i+1}^{\hbar} & =\widehat{G}_{i, i+1}^{\hbar}, & \\
\widetilde{\widehat{G}}_{i+1, i+1}^{\hbar} & =\widehat{G}_{i, i}^{\hbar}, & \\
\widetilde{\widehat{G}}_{i, i}^{\hbar} & =q \widehat{G}_{i, i+1}^{\hbar} \widehat{G}_{i, i}^{\hbar}-q^{2} \widehat{G}_{i+1, i+1}^{\hbar}=q^{-1} \widehat{G}_{i, i}^{\hbar} \widehat{G}_{i, i+1}^{\hbar}-q^{-2} \widehat{G}_{i+1, i+1}^{\hbar}, \\
\widetilde{\widehat{G}}_{i+1, i}^{\hbar} & =\widehat{G}_{i+1, i}^{\hbar}+\widehat{G}_{i, i}^{\hbar} \widehat{G}_{i, i+1}^{\hbar} \widehat{G}_{i, i}^{\hbar}-q^{-1} \widehat{G}_{i+1, i+1}^{\hbar} \widehat{G}_{i, i}^{\hbar}-q \widehat{G}_{i, i}^{\hbar} \widehat{G}_{i+1, i+1}^{\hbar},
\end{array}
$$


и для $\beta_{n, 1}^{\hbar}$ имеем:

$$
\begin{array}{ll}
\widetilde{\widehat{G}}_{1, k}^{\hbar}=\widehat{G}_{n, k}^{\hbar}, & k \neq n, 1, \\
\widetilde{\widehat{G}}_{n, k}^{\hbar}=q \widehat{G}_{n, 1}^{\hbar} \widehat{G}_{n, k}^{\hbar}-q^{2} \widehat{G}_{1, k}^{\hbar}=q^{-1} \widehat{G}_{n, k}^{\hbar} \widehat{G}_{n, 1}^{\hbar}-q^{-2} \widehat{G}_{1, k}^{\hbar}, & k \neq n, 1, \\
\widetilde{\widehat{G}}_{k, 1}^{\hbar}=\widehat{G}_{k, n}^{\hbar}, & k \neq n, 1, \\
\widetilde{G}_{k, n}^{\hbar}=q \widehat{G}_{n, 1}^{\hbar} \widehat{G}_{k, n}^{\hbar}-q^{2} \widehat{G}_{k, 1}^{\hbar}=q^{-1} \widehat{G}_{k, n}^{\hbar} \widehat{G}_{n, 1}^{\hbar}-q^{-2} \widehat{G}_{k, 1}^{\hbar}, & k \neq n, 1, \\
\widetilde{\widehat{G}}_{n, 1}^{\hbar}=\widehat{G}_{n, 1}^{\hbar}, & \\
\widetilde{\widehat{G}}_{1,1}^{\hbar}=\widehat{G}_{n, n}^{\hbar}, & \\
\widetilde{G}_{n, n}^{\hbar}=q \widehat{G}_{n, 1}^{\hbar} \widehat{G}_{n, n}^{\hbar}-q^{2} \widehat{G}_{1,1}^{\hbar}=q^{-1} \widehat{G}_{n, n}^{\hbar} \widehat{G}_{n, 1}^{\hbar}-q^{-2} \widehat{G}_{1,1}^{\hbar}, & \\
\widetilde{\widehat{G}}_{1, n}^{\hbar}=\widehat{G}_{1, n}^{\hbar}+\widehat{G}_{n, n}^{\hbar} \widehat{G}_{n, 1}^{\hbar} \widehat{G}_{n, n}^{\hbar}-q^{-1} \widehat{G}_{1,1}^{\hbar} \widehat{G}_{n, n}^{\hbar}-q \widehat{G}_{n, n}^{\hbar} \widehat{G}_{1,1}^{\hbar} . &
\end{array}
$$

Лемма 9.7. Следующие четыре матрицы с операторнозначными элементами, равно как и любая их линейная комбинация, преобразуются в соответствии с законом преобразования (84) квантовой подгруппы $A_{n}$ преобразований полной квантовой группы кос: $\widehat{\mathscr{A}}^{\hbar}$ вида (81) со всеми элементами $G_{i, j}^{\hbar}$ в верхнетреугольной части, замененными на элементы $\widehat{G}_{i, j}^{\hbar},\left(\widehat{\mathscr{A}}^{\hbar}\right)^{\dagger}, \widehat{\mathscr{R}}^{\hbar} u \widehat{\mathscr{S}}^{\hbar}$, где

$$
\begin{aligned}
& \left(\widehat{\mathscr{R}}^{\hbar}\right)_{i, j}=\left\{\begin{array}{ll}
-\widehat{G}_{j, i}^{\hbar}-q^{2} \widehat{G}_{i, j}^{\hbar}+q \widehat{G}_{i, i}^{\hbar} \widehat{G}_{j, j}^{\hbar}, & j>i, \\
\widehat{G}_{i, j}^{\hbar}+q^{-2} \widehat{G}_{j, i}^{\hbar}-q^{-1} \widehat{G}_{i, i}^{\hbar} \widehat{G}_{j, j}^{\hbar}, & j<i, \\
0, & j=i,
\end{array} \quad\left(\widehat{\mathscr{R}}^{\hbar}\right)^{\dagger}=-\widehat{\mathscr{R}}^{\hbar},\right. \\
& \left(\widehat{\mathscr{S}}^{\hbar}\right)_{i, j}=\widehat{G}_{i, i}^{\hbar} \widehat{G}_{j, j}^{\hbar}, \quad 1 \leqslant i, j \leqslant n, \quad\left(\widehat{\mathscr{S}}^{\hbar}\right)^{\dagger}=\widehat{\mathscr{S}}^{\hbar} .
\end{aligned}
$$

Чтобы представить вышеприведенное действие группы кос в матричном виде, обратимся к конструкции, предложенной в работе [6]. Применяя теорему 8.3, построим сначала конечномерное представление редуцированной на уровне четыре $\left(\Pi^{2}=2\right)$ алгебры, введя матрицы размера $(4 n) \times(4 n)$ с квантовыми элементами. Такие $((4 n) \times(4 n))$-матрицы $\mathbb{B}_{i, i+1}^{\hbar}$ при $1 \leqslant i \leqslant n-1$ имеют блочно-диагональный вид, в котором диагональные составляющие представляют собой $(n \times n)$-матрицы $B_{i, i+1}^{\hbar}(83)$, а недиагональные матрицы - нулевые, в то время как оставшаяся матрица $\mathbb{B}_{n, 1}^{\hbar}(\lambda)$ имеет вид

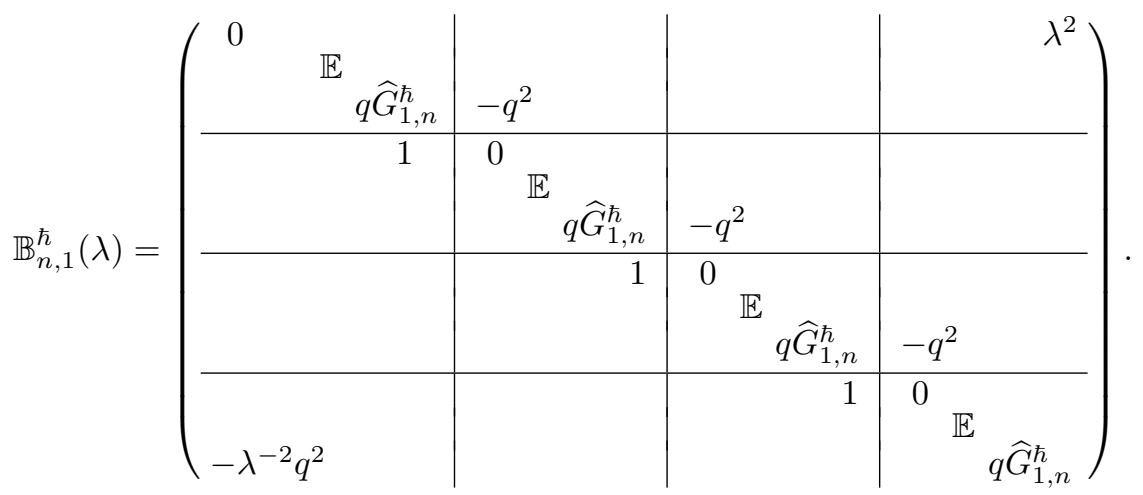


Вводя квантовую матрицу

$$
\mathbb{A}^{\hbar}=\left[\begin{array}{cccc}
\widehat{\mathscr{A}}^{\hbar} & \widehat{B}^{\hbar} & \widehat{C}^{\hbar} & \left(\widehat{B}^{\hbar}\right)^{\dagger} \\
0 & \widehat{\mathscr{A}}^{\hbar} & \widehat{B}^{\hbar} & \widehat{C}^{\hbar} \\
0 & 0 & \widehat{\mathscr{A}}^{\hbar} & \widehat{B}^{\hbar} \\
0 & 0 & 0 & \widehat{\mathscr{A}}^{\hbar}
\end{array}\right],
$$

где

$$
\begin{aligned}
& \widehat{B}^{\hbar}=\widehat{\mathscr{R}}^{\hbar}+q^{-1} \widehat{\mathscr{S}}^{\hbar}+q^{2} \widehat{\mathscr{A}}^{\hbar}-q^{-2}\left(\widehat{\mathscr{A}}^{\hbar}\right)^{\dagger}, \\
& \widehat{C}^{\hbar}=\left(q+q^{-1}\right) \widehat{\mathscr{S}}^{\hbar}-\widehat{\mathscr{A}}^{\hbar}-\left(\widehat{\mathscr{A}}^{\hbar}\right)^{\dagger},
\end{aligned}
$$

можно задать представление квантовой группы кос.

Теорема 9.8. Соотношения квантовой группь кос (90) и (91) можно представить в виде матричных соотношений на матрицу $\lambda \mathbb{A}^{\hbar}+\lambda^{-1}\left(\mathbb{A}^{\hbar}\right)^{\dagger}$, где матрица $\mathbb{A}^{\hbar}$ задается соотношениями (93), (94):

$$
\begin{aligned}
\beta_{i, i+1}^{\hbar}\left(\lambda \mathbb{A}^{\hbar}+\lambda^{-1}\left(\mathbb{A}^{\hbar}\right)^{\dagger}\right) & =\mathbb{B}_{i, i+1}^{\hbar}\left(\lambda \mathbb{A}^{\hbar}+\lambda^{-1}\left(\mathbb{A}^{\hbar}\right)^{\dagger}\right)\left(\mathbb{B}_{i, i+1}^{\hbar}\right)^{\dagger}, \quad i=1, \ldots, n-1, \\
\beta_{n, 1}^{\hbar}\left(\lambda \mathbb{A}^{\hbar}+\lambda^{-1}\left(\mathbb{A}^{\hbar}\right)^{\dagger}\right) & =\mathbb{B}_{n, 1}^{\hbar}(\lambda)\left(\lambda \mathbb{A}^{\hbar}+\lambda^{-1}\left(\mathbb{A}^{\hbar}\right)^{\dagger}\right)\left(\mathbb{B}_{n, 1}^{\hbar}\left(\lambda^{-1}\right)\right)^{\dagger},
\end{aligned}
$$

и в котором $\mathbb{B}_{i, i+1}^{\hbar}$ имеет блочно-диагональный вид с блоками вида (83) на диагонали, а $\mathbb{B}_{n, 1}^{\hbar}(\lambda)$ имеет вид (92).

С помощью прямого вычисления можно доказать справедливость следствия из этой теоремы.

СледСтвиЕ 9.9. В соответствии с представлением (67) возъмем (конечную) сумму

$$
\widehat{\mathscr{A}}^{\hbar}+\lambda^{-1} \widehat{\mathscr{B}}^{\hbar}+\lambda^{-2} \widehat{\mathscr{C}}^{\hbar}+\lambda^{-3}\left(\widehat{\mathscr{B}}^{\hbar}\right)^{\dagger}+\lambda^{-4}\left(\widehat{\mathscr{A}}^{\hbar}\right)^{\dagger}=\lambda^{-3}(1+\lambda) \widehat{\mathscr{G}}^{\hbar}(\lambda),
$$

где $\widehat{\mathscr{G}}^{\hbar}(\lambda)-$ матрица размера $n \times n$ вида:

$\widehat{\mathscr{G}}^{\hbar}(\lambda):=(\lambda-1)\left(\lambda+q^{2}\right) \widehat{\mathscr{A}}^{\hbar}-\left(1-\lambda^{-1}\right)\left(q^{-2} \lambda+1\right)\left(\widehat{\mathscr{A}}^{\hbar}\right)^{\dagger}+(\lambda-1) \widehat{\mathscr{R}}^{\hbar}+\left(q^{-1} \lambda+q\right) \hat{\mathscr{S}}^{\hbar}$.

Тогда матрица $\widehat{\mathscr{G}}^{\hbar}(\lambda)$ преобразуется в соответствии с матричным представлением (86), (87) квантовой группы кос.

\section{0. Фробениусовы многообразия в окрестности неполупростой точки}

В этом разделе алгебра $\mathfrak{D}_{n}$ интерпретируется как пуассонова алгебра для данных Стокса на фробениусовом многообразии в окрестности неполупростой точки. 
10.1. Слияние полюсов для фробениусова многообразия. Фробениусовы многообразия были введены Б.А. Дубровиным [11] для бескоординатного описания известных соотношений Виттена-Дойкграфа-Г. ВерлиндеЭ. Верлинде (ВДВВ). Фробениусова алгебра представляет собой коммутативную ассоциативную алгебру с единицей с инвариантной невырожденной билинейной формой (имеются и другие ограничения, см. строгое определение в работе [11]).

Полупростое фробениусово многообразие размерности $n$ можно реализовать на пространстве параметров $\mathbf{u}=\left(u_{1}, \ldots, u_{n}\right)$ вместе с антисимметричной $(n \times n)$-матричнозначной функцией $V(\mathbf{u})$ такой, что линейный дифференциальный оператор

$$
\Lambda(z):=\frac{\mathrm{d}}{\mathrm{dz}}-U-\frac{V(\mathbf{u})}{z},
$$

где $U$ - диагональная матрица с элементами $u_{1}, \ldots, u_{n}$, имеет инвариантные данные монодромии [11]. Обычно данные монодромии оператора $\Lambda(z)$ представляются в виде матрицы Cтокса $S$, представляющей собой верхнетреугольную матрицу с единицами на диагонали (общее определение данных монодромии см. в работах [34]-[36]). Эквивалентно, полупростые фробениусовы многообразия можно отождествлять с пространством изомонодромных деформаций $n$-мерной фуксовой системы ${ }^{10}$ :

$$
\frac{\mathrm{d} \Psi}{\mathrm{d} z}=\sum_{k=1}^{n} \frac{\mathscr{A}_{k}(\mathbf{u})}{\lambda-u_{k}} \Psi
$$

где матрицы $\mathscr{A}_{1}(\mathbf{u}), \ldots, \mathscr{A}_{n}(\mathbf{u})$ имеют вид

$$
\mathscr{A}_{k}(\mathbf{u})=-E_{k, k}\left(V(\mathbf{u})+\frac{1}{2} \mathbb{E}\right), \quad\left(E_{k, k}\right)_{i, j}=\delta_{i k} \delta_{k j}, \quad k=1, \ldots, n .
$$

Пусть $S$ - матрица Стокса дифференциального оператора $\Lambda(z):=\frac{\mathrm{d}}{\mathrm{d} z}-U-\frac{V}{z}$. Если $\operatorname{rank} G=n$, где $G=S+S^{T}$, то матрицы монодромий системы (95) имеют вид

$$
\mathscr{M}_{k}=\mathbb{E}-E_{k, k}\left(S+S^{T}\right)=\mathbb{E}-E_{k, k} G, \quad k=1, \ldots, n .
$$

Применяя скобку Короткина-Самтлебена и соотношение

$$
\operatorname{Tr}\left(\mathscr{M}_{i} \mathscr{M}_{j}\right)=n-4+S_{i, j}^{2},
$$

Моника Угалья построила скобку Пуассона на множестве элементов $S_{i, j}$ матрицы Стокса [7]. Ею были получены те же самые выражения (16) с элементами $S_{i, j}$ вместо $G_{i, j}$ (с точностью до множителя $\left.-1 / 2\right)$.

То, что алгебра $\mathfrak{D}_{n}$ допускает интерпретацию алгебры Пуассона на множестве данных Стокса для фробениусова многообразия в окрестности неполупростой точки, следует из того факта, что такие точки отвечают критическим точкам уравнений Шлезингера, т. е. точкам, в которых сталкиваются два или более полюсов фуксовой системы (95).

\footnotetext{
${ }^{10}$ Каллиграфический шрифт используется, чтобы отличать системы размера $n \times n$ с их матрицами монодромий от систем размера $2 \times 2$ с соответствующими матрицами монодромий, рассматриваемых в предыдущих разделах.
} 
Будем использовать те же обозначения, что и в п. 6.3. В частности, зафиксируем целое число $\tilde{n}$ и положим

$$
\widetilde{\mathbf{u}}:=\left(u_{1}, \ldots, u_{\tilde{n}-1}\right), \quad u_{j}:=t v_{j}, \quad j=\tilde{n}, \ldots, \tilde{n}+m-1=n .
$$

Определим теперь $A_{i}(\widetilde{\mathbf{u}}, t)$ при $i=1, \ldots, \tilde{n}-1$ и $B_{j}(\widetilde{\mathbf{u}}, t)$ при $j=1, \ldots, m=$ $n-\tilde{n}+1$ так же, как и в формуле (37).

ТеОРема 10.1 [9]. Предположим, что $V$ - нерезонансная матрица и что ее собственные значения $\mu_{1}, \ldots, \mu_{n}$ имеют вещественные части $\operatorname{Re} \mu_{i} \in$ $(-1 / 2,1 / 2)$. Если $\operatorname{rank}\left(S+S^{T}\right)=n$, то существуют матричнозначные функиии $\mathscr{A}_{1}^{0}(\widetilde{\mathbf{u}}), \ldots, \mathscr{A}_{\tilde{n}-1}^{0}(\widetilde{\mathbf{u}})$ и $\mathscr{B}_{1}^{0}(\widetilde{\mathbf{u}}), \ldots, \mathscr{B}_{n-\tilde{n}+1}^{0}(\widetilde{\mathbf{u}})$ такие, что фуксова система

$$
\frac{\mathrm{d} \widetilde{\Phi}}{\mathrm{d} z}=\sum_{k=1}^{\tilde{n}} \frac{\widetilde{\mathscr{A}}_{k}}{\lambda-u_{k}} \widetilde{\Phi}
$$

$c$

$$
u_{\tilde{n}}=0, \quad \tilde{\mathscr{A}}_{i}=\mathscr{A}_{i}^{0}, \quad i=1, \ldots, \tilde{n}-1, \quad \tilde{\mathscr{A}}_{\tilde{n}}=\sum_{j=1}^{n-\tilde{n}+1} \mathscr{B}_{j}^{0}
$$

имеет матрицы монодромий $\mathscr{M}_{1}, \ldots, \mathscr{M}_{\tilde{n}-1}, \mathscr{M}_{h}$, где

$$
\mathscr{M}_{h}=\mathscr{M}_{\tilde{n}} \mathscr{M}_{\tilde{n}+1} \cdots \mathscr{M}_{n} .
$$

У этой теоремы имеется важное следствие.

ТЕОРема 10.2. Пуассонова алгебра данных монодромии системъ (98) есть алгебра $\mathfrak{D}_{n}$.

ДокАЗАТЕЛЬство. Пуассонова алгебра данных монодромии системы (98) задается скобкой Короткина-Самтлебена, суженной на инвариантные относительно присоединенного действия функции, такие как

$$
\operatorname{Tr}\left(\mathscr{M}_{i} \mathscr{M}_{j}\right) \quad \text { и } \operatorname{Tr}\left(\mathscr{M}_{i} \mathscr{M}_{h}^{k} \mathscr{M}_{j} \mathscr{M}_{h}^{-k}\right),
$$

где теперь матрицы монодромий имеют размер $n \times n$. В силу (97) легко доказать, что

$\operatorname{Tr}\left(\mathscr{M}_{i} \mathscr{M}_{h}^{k} \mathscr{M}_{j} \mathscr{M}_{h}^{-k}\right)=\operatorname{Tr}\left(\mathscr{M}_{j}-E_{i, i} G+E_{i, i} G \mathscr{M}_{h}^{k} E_{j, j} G \mathscr{M}_{h}^{-k}\right)=n-4+\left(G \mathscr{M}_{h}^{k}\right)_{i, j}^{2}$,

где последнее действие можно выполнить ввиду тождества $G \mathscr{M}_{h}=\mathscr{M}_{h}^{-T} G$, которое является прямым следствием условий (97) и (99). Определяя

$$
G_{i, j}^{(k)}=\left(G \mathscr{M}_{h}^{k}\right)_{i, j}
$$

получим, что скобки Пуассона между элементами $G_{i, j}^{(k)}$ совпадают с $(43)$ с точностью до множителя $-1 / 2$. Теорема 10.2 доказана.

\section{2. Редукции уровня $p$ в случае фробениусовых многообразий.} Исследуем теперь вопрос о том, какие ограничения следует наложить на элементы $G_{i, j}, i, j=1, \ldots, n$, чтобы обеспечить выполнение условий редукции уровня $p: \mathscr{M}_{h}^{p}=\mathbb{E}(65)$. 
ПрЕДЛОЖЕНИЕ 10.3. Произведение матрии монодромий

$$
\mathscr{M}_{h}:=\mathscr{M}_{\tilde{n}} \mathscr{M}_{\tilde{n}+1} \cdots \mathscr{M}_{n}
$$

имеет следующую блочно-матричную структуру:

$$
\mathscr{M}_{h}=\left[\begin{array}{c|c}
\mathbb{E}_{(\tilde{n}-1) \times(\tilde{n}-1)} & \mathbb{O} \\
\hline B & -\widetilde{S}^{-1} \widetilde{S}^{T}
\end{array}\right],
$$

где $B$ - матрица размера $(n-\tilde{n}+1) \times(\tilde{n}-1)$, элементы которой являются многочленами от $G_{i, j}$ c $i=1, \ldots, n u j=\tilde{n}, \ldots, n$, а матрица $\widetilde{S}$ есть верхнетреугольная $((n-\tilde{n}+1) \times(n-\tilde{n}+1))$-матрица с единицами на диагонали и с элементами $G_{\tilde{n}+i-1, \tilde{n}+j-1}$ на $(i, j)$-месте над диагональю.

ДокАЗАТЕЛьСтво. Из явного вида матрицы $\mathscr{M}_{i}(97)$ следует, что только последние $n-\tilde{n}+1$ рядов матрицы $\mathscr{M}_{h}$ отличают ее от единичной матрицы и что только те $G_{i, j}$, у которых оба индекса $i$ и $j$ больше или равны $\tilde{n}$, дают вклад в выражение в правом нижнем квадратном блоке; мы обозначим через $\widetilde{\mathscr{M}}_{r} \mathrm{c} r=$ $\tilde{n}, \ldots, n$ правые нижние $((n-\tilde{n}+1) \times(n-\tilde{n}+1))$-блоки соответствующих матриц монодромий $\mathscr{M}_{r}$. Утверждение предложения при этом следует из тождества Дубровина $\widetilde{\mathscr{M}}_{\tilde{n}} \widetilde{\mathscr{M}}_{\tilde{n}+1} \cdots \widetilde{\mathscr{M}}_{n}=-\widetilde{S}^{-1} \widetilde{S}^{T} \cdot{ }^{11}$ Предложение 10.3 доказано.

Введем теперь обозначение $\widetilde{\mathscr{M}}_{h}:=-\widetilde{S}^{-1} \widetilde{S}^{T}$. Достаточные условия содержатся в следующей лемме.

Лемма 10.4. Условие (65) выполняется, если $\left(\widetilde{\mathscr{M}}_{h}\right)^{p}=\mathbb{E}$, а симметрическая форма $\widetilde{S}+\widetilde{S}^{T}$ невырождена.

ДокАЗАТЕЛЬСтво. Из явного вида $\mathscr{M}_{h}$ ясно, что условие (65) эквивалентно одновременному выполнению двух условий:

(i) $\left(\mathbb{E}+\widetilde{\mathscr{M}}_{h}+\left(\widetilde{\mathscr{M}}_{h}\right)^{2}+\cdots+\left(\widetilde{\mathscr{M}}_{h}\right)^{p-1}\right) B=0$ и

(ii) $\left(\widetilde{\mathscr{M}}_{h}\right)^{p}=\mathbb{E}$.

Умножив равенство в (i) на $\left(\widetilde{\mathscr{M}}_{h}-\mathbb{E}\right)$ и применяя второе условие, придем к тождеству. Невырожденность матрицы $\left(\widetilde{\mathscr{M}}_{h}-\mathbb{E}\right)$ предполагает выполнение первого условия. Но это условие невырожденности является в точности условием невырожденности симметрической формы $\widetilde{S}+\widetilde{S}^{T}$ (после умножения на $\widetilde{S}$ справа). Лемма 10.4 доказана.

Авторы признательны Алексею Бондалу, Борису Дубровину, Владимиру Фоку, Давиду Гуззетти, Найджелу Хитчину, Дмитрию Короткину, Александру Молеву, Максиму Назарову, Роберту Пеннеру, Василисе Шрамченко, Александру Строхмайеру и Александру Веселову за многочисленные плодотворные обсуждения.

\footnotetext{
${ }^{11}$ Напомним, что сама эта формула является следствием цепочки матричных равенств: $S E_{1,1}=E_{1,1}, E_{i, i} S^{T} E_{j, j}=0$ при $i<j, E_{i, i} S E_{j, j}=\delta_{i, j} E_{j, j}$ при $i \geqslant j$; тогда $S \mathscr{M}_{1}=S-$ $E_{1,1}\left(S+S^{T}\right)=\left(E_{2,2}+\cdots+E_{n, n}\right) S-E_{1} S^{T}$. Умножив это выражение на $\mathscr{M}_{2}$ справа и применяя вышеприведенные формулы, получим в результате $\left(E_{3,3}+\cdots+E_{n, n}\right) S-\left(E_{1,1}+E_{2,2}\right) S^{T}$; умножим полученное выражение на $\mathscr{M}_{3}$ справа и продолжим этот процесс до тех пор, пока мы не получим, что $S \mathscr{M}_{1} \mathscr{M}_{2} \cdots \mathscr{M}_{n}=-\left(E_{1,1}+E_{2,2}+\cdots+E_{n, n}\right) S^{T}=-S^{T}$.
} 


\section{Список литературы}

[1] E. Verlinde, H. Verlinde, "Conformal field theory and geometric quantization", Superstrings'89 (Trieste, 1989), World Sci. Publ., River Edge, NJ, 1990, 422-449.

[2] J. E. Nelson, T. Regge, "Homotopy groups and (2+1)-dimensional quantum gravity", Nuclear Phys. B, 328:1 (1989), 190-202.

[3] J. E. Nelson, T. Regge, F. Zertuche, "Homotopy groups and (2+1)-dimensional quantum de Sitter gravity", Nuclear Phys. B, 339:2 (1990), 516-532.

[4] L. O. Chekhov, V. V. Fock, "Observables in 3D gravity and geodesic algebras", Quantum groups and integrable systems, Czechoslovak J. Phys., 50:11 (2000), 1201-1208.

[5] L. O. Chekhov, R. C. Penner, "On quantizing Teichmüller and Thurston theories", Handbook on Teichmüller Theory, Vol. I, IRMA Lect. Math. Theor. Phys., 11, Eur. Math. Soc., Zürich, 2007, 579-646.

[6] L. O. Chekhov, "Orbifold Riemann surfaces and geodesic algebras", J. Phys. A, 42 (2009), Paper 304007.

[7] M. Ugaglia, "On a Poisson structure on the space of Stokes matrices", Internat. Math. Res. Notices, 1999:9 (1999), 473-493; arXiv: math/9902045.

[8] А.И. Бондал, "Симплектический группоид треугольных билинейных форм и группа кос", Изв. РАН. Сер. матем., 68:4 (2004), 19-74; англ. пер.: А. I. Bondal, "A symplectic groupoid of triangular bilinear forms and the braid group", Izv. Math., 68:4 (2004), 659-708.

[9] L. O. Chekhov, M. Mazzocco, Isomonodromic deformations and twisted Yangians arising in Teichmüller theory, arXiv: math/0909.5350, 2009.

[10] A. I. Molev, E. Ragoucy, P. Sorba, "Coideal subalgebras in quantum affine algebras", Rev. Math. Phys., 15:8 (2003), 789-822.

[11] B. Dubrovin, "Geometry of 2D topological field theories", Integrable systems and quantum groups (Montecatini Terme, 1993), Lecture Notes in Math., 1620, Springer, Berlin, 1996, 120-348.

[12] L. O. Chekhov, "Teichmüller theory of bordered surfaces", SIGMA Symmetry Integrability Geom. Methods Appl., 3 (2007), Paper 066.

[13] A.I. Molev, E. Ragoucy, "Symmetries and invariants of twisted quantum algebras and associated Poisson algebras", Rev. Math. Phys., 20:2 (2008), 173-198.

[14] W. M. Goldman, "Invariant functions on Lie groups and Hamiltonian flows of surface group representations", Invent. Math., 85:2 (1986), 263-302.

[15] D. Korotkin, H. Samtleben, "Quantization of coset space $\sigma$-models coupled to two-dimensional gravity", Comm. Math. Phys., 190:2 (1997), 411-457.

[16] V.V. Fock, Description of moduli space of projective structures via fat graphs, arxiv.org/abs/hep-th/9312193.

[17] Л. О. Чехов, "Римановы поверхности с орбифолдными точками", Геометрия, топология и математическая физика. II. K 70-летию со дня рождения академика Сергея Петровича Новикова, Тр. МИАН, 266, МАИК, М., 2009, 237-262; англ. пер.: L. O. Chekhov, "Riemann surfaces with orbifold points", Proc. Steklov Math. Inst., 266:1 (2009), 228-250.

[18] R. C. Penner, "The decorated Teichmüller space of Riemann surfaces", Comm. Math. Phys., 113:2 (1988), 299-339.

[19] V.V. Fock, A. B. Goncharov, "Dual Teichmüller and lamination spaces", Handbook of Teichmüller theory, Vol. I, IRMA Lect. Math. Theor. Phys., 11, Eur. Math. Soc., Zürich, 2007, 647-684; arXiv: math/0510312, 2005.

[20] V.V. Fock, Dual Teichmüller spaces, arXiv: dg-ga/9702018, 1997.

[21] W.P. Thurston, Minimal stretch maps between hyperbolic surfaces, arXiv: math/ 9801039, 1998. 
[22] F. Bonahon, "Shearing hyperbolic surfaces, bending pleated surfaces and Thurston's symplectic form", Ann. Fac. Sci. Toulouse Math. (6), 5:2 (1996), 233-297.

[23] S. Fomin, A. Zelevinsky, "Cluster algebras. I. Foundations", J. Amer. Math. Soc., 15:2 (2002), 497-529; arXiv: math/0104151, 2001.

[24] S. Fomin, A. Zelevinsky, The Laurent phenomenon, arXiv: math/0104241, 2001.

[25] В. В. Фок, Л. О. Чехов, "Квантовые пространства Тейхмюллера", ТМФ, 120:3 (1999), 511-528; англ. пер.: V. V. Fock, L. O. Chekhov, "A quantum Teichmüller space", Theoret. and Math. Phys., 120:3 (1999), 1245-1259; arXiv: math/9908165, 1999.

[26] В. В. Фок, Л. О. Чехов, "Квантовые модулярные преобразования, соотношение пятиугольника и геодезические", Математическая физика. Проблемы квантовой теории поля. K 65-летию со дня рождения академика Людвига Дмитриевича Фаддеева, Тр. МИАН, 226, Наука, М., 1999, 163-179; англ. пер.: V. V. Fock, L. O. Chekhov, "Quantum mapping class group, pentagon relation, and geodesics", Proc. Steklov Math. Inst., 226 (1999), 149-163.

[27] S. Fomin, M. Shapiro, D. Thurston, "Cluster algebras and triangulated surfaces. I: Cluster complexes", Acta Math., 2008, № 1, 83-146; arXiv: math/0608367, 2006.

[28] W. Dekkers, "The matrix of a connection having regular singularities on a vector bundle of rank 2 on $P^{1}(C)$ ", Équations différentielles et systèmes de Pfaff dans le champ complexe (Sem., Inst. Rech. Math. Avancée, Strasbourg, 1975), Lecture Notes in Math., 712, Springer, Berlin, 1979, 33-43.

[29] B. Malgrange, "Sur les déformations isomonodromiques. I: Singularités régulières", Mathematics and physics (Paris, 1979-1982), Progr. Math., 37, Birkhäuser, Boston, MA, 1983, 401-426.

[30] T. Miwa, "Painlevé property of monodromy preserving deformation equations and the analyticity of $\tau$-function", Publ. Res. Inst. Math. Sci., 17:2 (1981), 703-721.

[31] B. Dubrovin, M. Mazzocco, "Monodromy of certain Painlevé-VI transcendents and reflection group", Invent. Math., 141:1 (2000), 55-147.

[32] M. Sato, T. Miwa, M. Jimbo, "Holonomic quantum fields. II: The Riemann-Hilbert problem", Publ. Res. Inst. Math. Sci., 15:1 (1979), 201-278.

[33] J. Teschner, "An analog of a modular functor from quantized Teichmüller theory", Handbook of Teichmüller theory, Vol. I, IRMA Lect. Math. Theor. Phys., 11, Eur. Math. Soc., Zürich, 2007, 685-760; arXiv: math/0510174, 2005.

[34] M. Jimbo, T. Miwa, "Monodromy preserving deformations of linear ordinary differential equations with rational coefficients. II", Phys. D, 2:3 (1981), 407-448.

[35] M. Jimbo, T. Miwa, "Monodromy preserving deformations of linear ordinary differential equations with rational coefficients. III", Phys. D, 4:1 (1982), 26-46.

[36] M. Jimbo, T. Miwa, K. Ueno, "Monodromy preserving deformations of linear ordinary differential equations with rational coefficients. I", Phys. D, 2:2 (1981), 306-352.

М. Маззокко (M. Mazzocco)

Университет Лавборо, Великобритания

\section{Л. O. Чехов (L. O. Chekhov)}

Институт теоретической и экспериментальной

физики им. А. И. Алиханова;

Математический институт им. В. А. Стеклова РАН;

Лаборатория Понселе Независимого московского

университета

E-mail: chekhov@mi.ras.ru
Поступила в редакцию 10.11.2009 